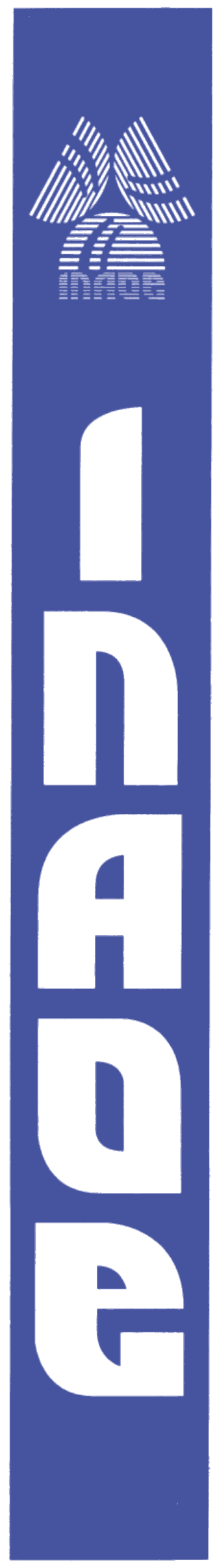

\title{
A New Fully Functioning Digital Hologram Recording System and its Applications
}

\author{
Por

\section{Miguel Alcaraz Rivera}

Tesis sometida como requisito parcial para obtener el grado de

\section{Doctor en Ciencias Especialidad en Óptica}

\author{
en el Instituto Nacional de Astrofísica, Óptica y \\ Electrónica.
}

Supervisado por

\section{Dr. José Javier Báez Rojas}

Dr. Der-Kuan Kang

Tonantzintla, Puebla.

$$
\text { Mayo } 2011
$$

OINAOE 2011

Derechos Reservados

El autor otorga al INAOE el permiso de reproducir y distribuir copias de esta tesis en su totalidad o en partes.

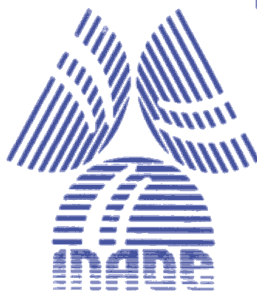




\section{Index}

A new fully functioning digital hologram recording system and its applications ......................... I

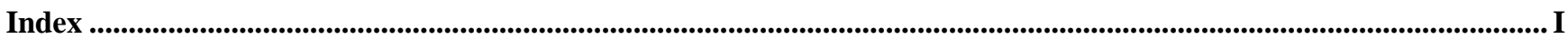

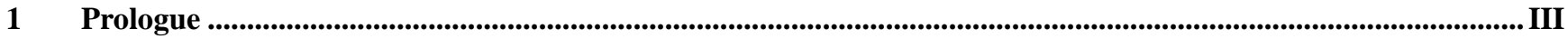

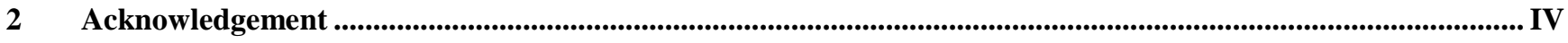

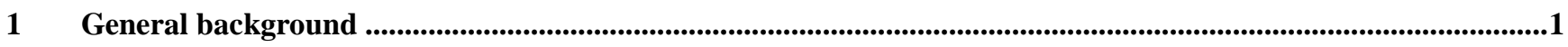

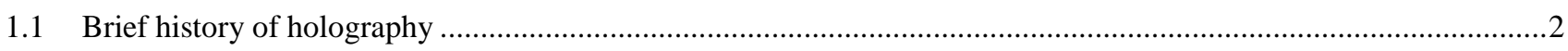

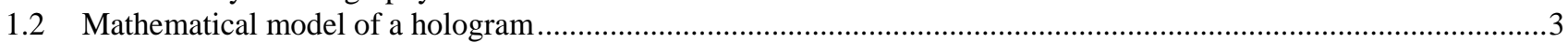

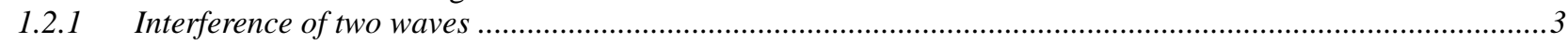

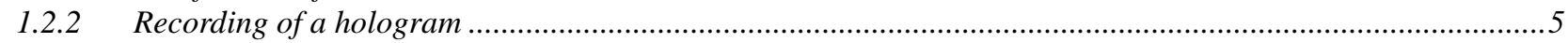

1.3 Hologram techniques particularly well suited for display applications ……...........................................................6

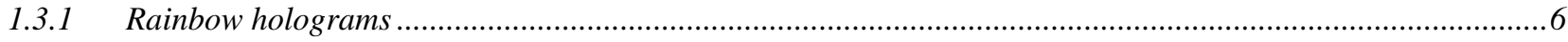

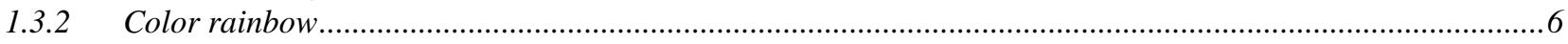

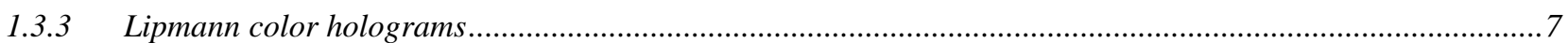

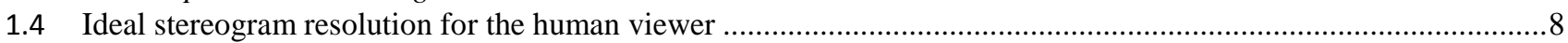

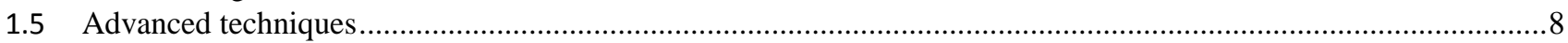

1.5.1 Dot-matrix

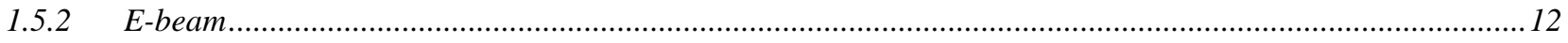

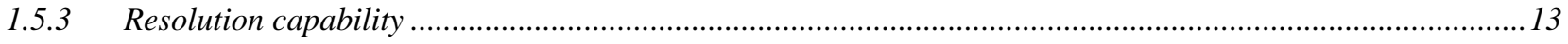

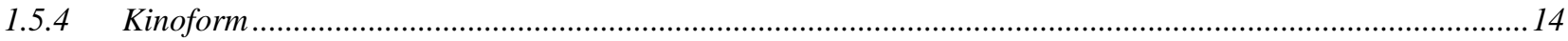

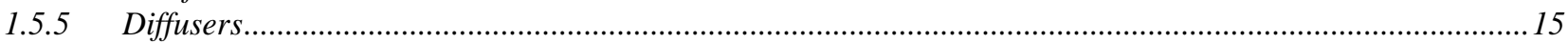

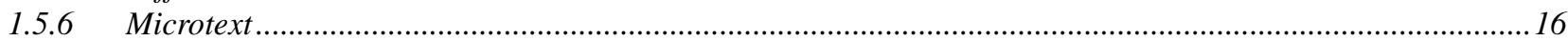

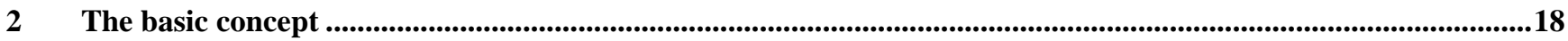

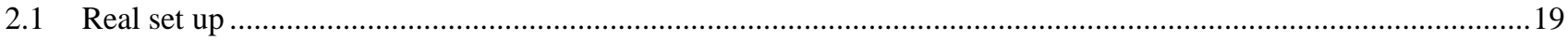

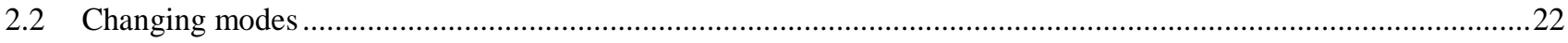

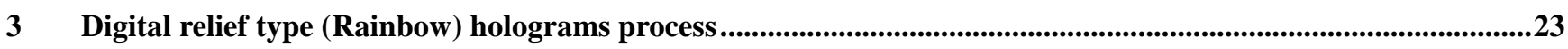

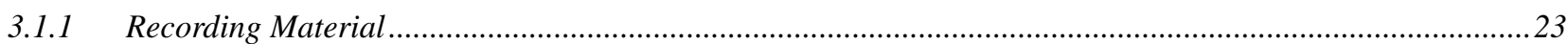

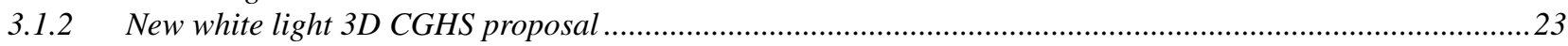

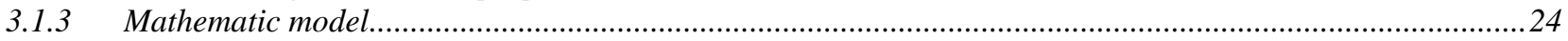

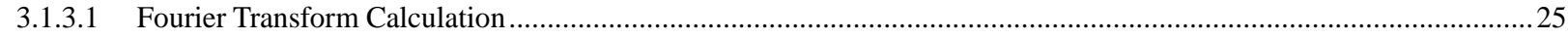

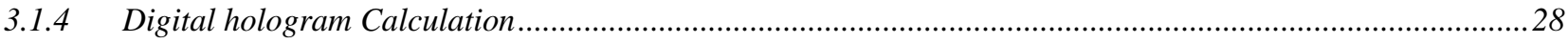

F1 .

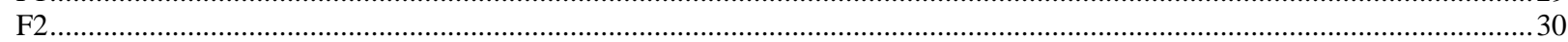

F4 (1) -

F6

F7

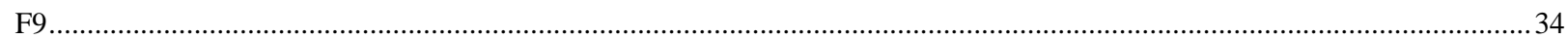

F10 (

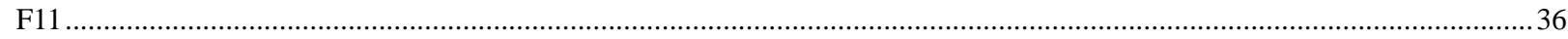

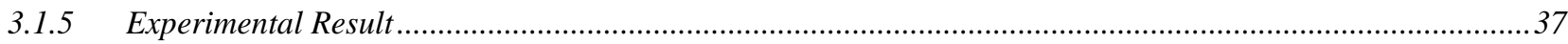

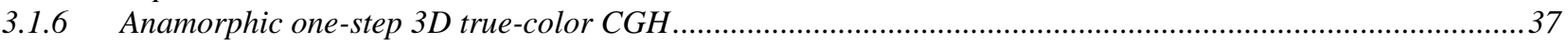

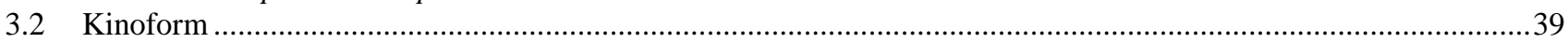

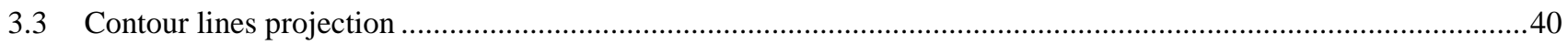

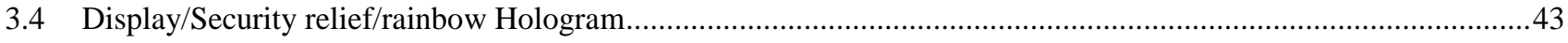

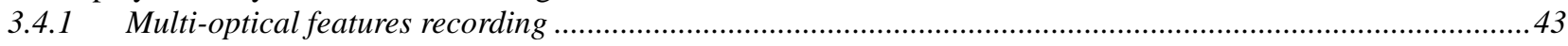

$4 \quad$ Lippmann holographic recording process .................................................................................................................46

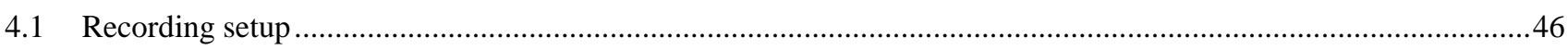

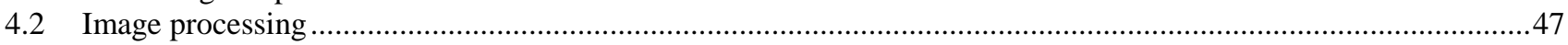

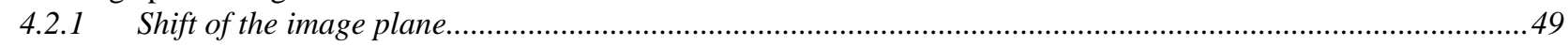

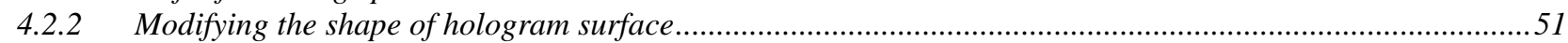




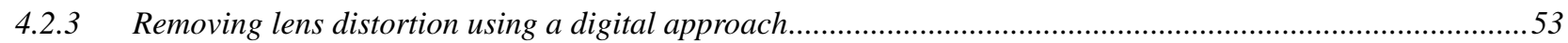

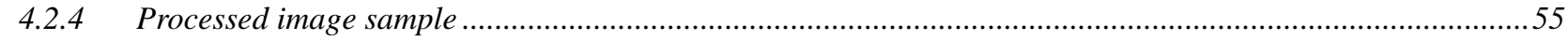

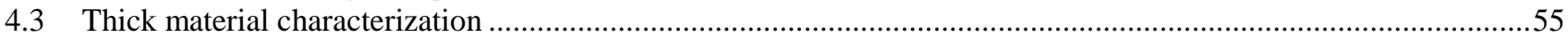

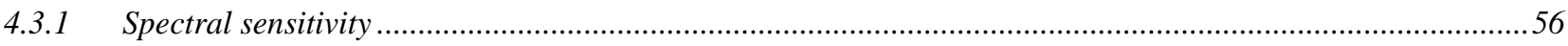

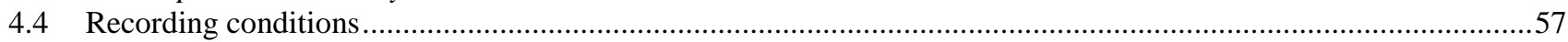

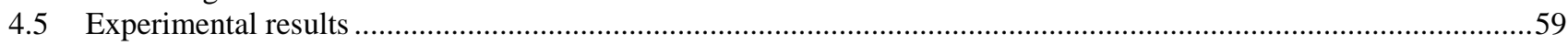

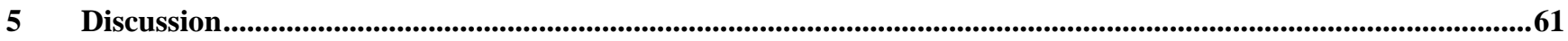

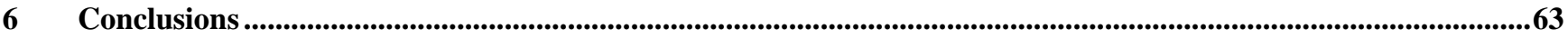

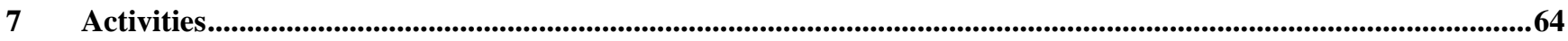

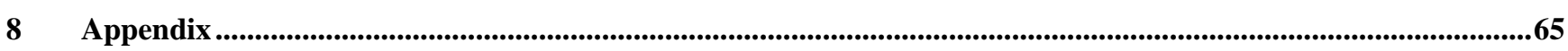

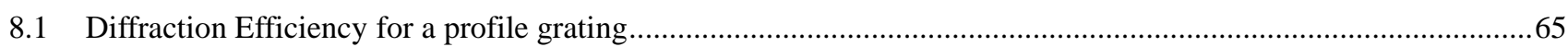

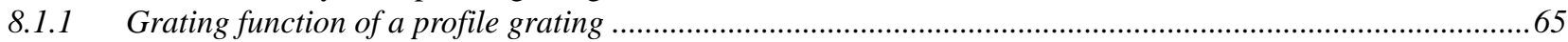

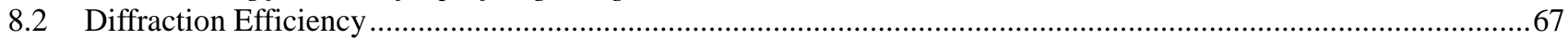

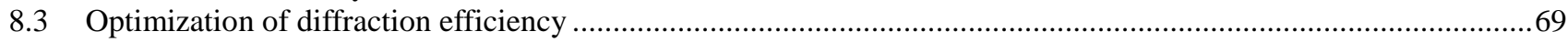

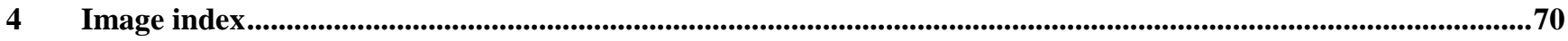

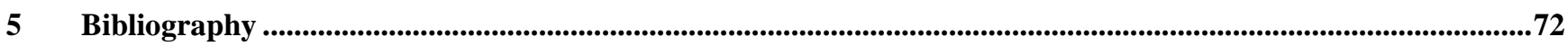




\section{Prologue}

The research described in this thesis is based on the digital hologram recording system which was proposed by Dr. DerKuan Kang in 2001. The basic system has capabilities of recording both of digital relief type (rainbow type) hologram and reflective type (Lippmann) hologram. This work was begun from 2008 in the laboratory located in New York under advised Dr. DerKuan Kang. This work focused on CGH and Lippmann hologram recording developments through enhancing software and optical setup on the basic system. 


\section{Acknowledgement}

I would like to thank the president of T-Security Inc., Taiwan, Mr. Hong Yang, Su for his support and encouragement during my studies in New York. My gratitude is also shared to Ms. Maria Luisa CruzLopez for her great job in part of CGH programming. Special thanks also for my teachers, DerKuan Kang and Dr. Javier Baez for their continuous support. Last, I would like to recognize CONACYT for the backing of all my work.

\section{谢谢你们！}




\section{General background}

This work will be focused on presenting the construction and achievability of a digital holography printing machine.

Holography has been, since its introduction with visual wavelengths, subject to continuous improvements and experimental setups, due to its visual appeal. A lot of these efforts have been very artistic, and as in every art, each one very personal and unique. A great deal of energies has been focused to mechanize the procedure and make it a full automated process, but there is always a tradeoff between achieving this and removing some of the qualities that make holograms so vivid and real. Some real effort has been put here, with lots of money backing up the results.

This can be seen in the general implementation of hologram security features in everyday products, like credit cards, some currencies, like euros, and a few merchandise labels. This kind of hologram use full advantage of techniques like rainbow holograms and other thin hologram techniques, and are very bright and difficult to replicate, and are also very cheap to print.

But a hologram with the quality of those big artistic holograms achieved in some college labs and private artist workshops is almost a complete world apart from the small and gaudy colored thin holograms applied for labeling.

In contrast to thin holograms, thick hologram techniques have been implemented and also automated in the past, but not as cheap as their thin counterparts. As will be discussed later in this work, the selective wavelength reconstruction that can be obtained in thick holograms can be used for a more precise color modeling, giving a more realistic result, getting closer to achieve a great realistic hologram.

An ideal hologram printing machine would be something similar to a photography printing machine, where one could select its image to print and seconds later obtain the result as a printed hologram. In present time, the input of a machine like this could be a digital 3D model that could have been obtained from a real life scene, or directly modeled in a computer environment. This model would be handled to the printer and printed over the surface chosen.

The dimensions of this ideal printer shouldn't be bigger than a photographic printer, and the price also shouldn't be unachievable. 
With this ideal machine as our objective, on this work we will build a holographic printing machine, and present our results and conclusions.

\subsection{Brief history of holography}

The vision is the sense that gives us more information about our surroundings. Since ever we have used visual representations to communicate information and have invented all kind of artifacts to record this ideas. All these techniques had one goal in common: to make light transfer information understandable to another human.

Holography is the perfect recording of a visual image. It allows the exact reconstruction of the light coming from a certain scene or object without the need of the original source being present.

The combination of holography with computers has created a series of technique that allows light manipulation and visual effects that still amaze and attract.

Holography started when Gabor applied the $\mathrm{X}$ ray interference photography technique to visible light and obtained a reconstructed image from an interference pattern previously photographed. This work was considered unimportant, and at first it was rejected for publishing for being unoriginal. (1).

But holographic applications started multiplying, and its use started inspiring people, not only on scientific fields, but also on the news and culture.

Very concise, optical holography time milestones can be described as: on axis holography, were the reconstruction had overlapped the reconstructed image, its conjugate, and the reference beam. Next, off axis holography, where the reference beam has an angle with the object beam, and this causes the spatial separation of the reconstructed image, its conjugate and the reference beam. Other milestones were white light reconstruction (2), Fourier holograms (G.W. Stroke), Fraunhoffer holograms (J.B.Develis), reflection (Denisyuk) holograms, color holograms, digital holograms and holographic optical elements, each one based on the same principle of holography, but applying different optical knowledge to improve the recording or the reconstruction.

Important to this work are the rainbow holograms and thick reflection type holograms. Rainbow holograms are recorded on two steps. The first step records a hologram with the basic technique, and on the second, the reconstruction of the first step with white light illumination passes through a thin slit and is then recorded again as a hologram. If this second step hologram is reconstructed, it will be visible on 
white light; these ideas will be revisited later. Lippmann holograms are holograms that use the principle of thick holograms to use white light as the light source in reconstructions and obtain an image in full color.

Holography has been a science limited by technology of its time. When Gabor proposed its method, the most efficient coherent light available was a mercury vapor lamp. It wasn't until the invention of the laser that real amazing results of three dimensional objects were obtained. Computer calculated holograms were also proposed before the result of those works could compare to their "analog" counterparts.

Right now, it is possible to calculate a complete interference pattern of a simulated hologram to record, but with unlimited computer power, even holographic movies could be possible.

Also, the display of the digital calculated holograms is limited with current technology, and displays capable of holographic resolution are very expensive and have limited pixel count. But as technology advances, holographic displays will dominate the 3D market with objects so real that will be actually indistinguishable from its solid versions.

\subsection{Mathematical model of a hologram}

A hologram is a recorded interference pattern. This pattern can be used to rebuild a copy of the original light beams that interfered. To explain the phenomena, we have to understand what an interference pattern is.

\subsubsection{Interference of two waves}

We can start with a plane wave propagating in $z$ direction

$$
E(z, t)=A \cos (2 \pi f t-k z+\varphi)=A \cos (\omega t-\Phi)
$$

where $\mathrm{A}$ is the amplitude of the oscillation. $\varphi$ represents a phase factor for time $t=0(2) . f$ is the frequency and $\mathrm{k}$ is the wave number. We introduce $\Phi$ as a phase containing the space information.

We can add an imaginary term with the same phase $i \sin (\omega t-\Phi)$ to Use Euler's relation so we can rewrite the wave as 


$$
E(z, t)=A e^{-i(\omega t-\Phi)}
$$

The temporal part of the equation will be averaged over multiple oscillations, thus. Can be ignored to obtain a complex wave:

$$
E(z, t)=A e^{-i \Phi}
$$

Given two waves called the object and the reference beam

$$
\begin{aligned}
& \mathbf{o}=o e^{-i \Phi} \\
& \mathbf{r}=r e^{-i \Psi}
\end{aligned}
$$

The amplitude of both in a point of space, is given by the superposition principle as $\mathbf{r}+\mathbf{o}$ and the intensity, the square of this

$$
\begin{gathered}
I=|\mathbf{r}+\mathbf{o}|^{2} \\
I=r r^{*}+o o^{*}+r o^{*}+o r^{*} \\
I=r^{2}+o^{2}+r o\left(e^{-i(\Phi-\Psi)}+e^{i(\Phi-\Psi)}\right) \\
I=r^{2}+o^{2}+r o \cos (\Phi-\Psi)
\end{gathered}
$$

If the phases vary independently, $\cos (\Phi-\Psi)$ averages 0 , and the intensity is always

$$
I=r^{2}+o^{2}
$$

But in the case $\Phi$ and $\Psi$ are related, the intensity in different points can vary with $\cos (\Phi-\Psi)$ taking values between -1 and +1 , and the two light sources are called coherent. 
The variation of the phase along the space is seen over a plane as a series of fringes of darker and lighter zones.

\subsubsection{Recording of a hologram}

The intensity distribution of an interference pattern $I(x, y)=|\mathbf{r}(x, y)+\mathbf{o}(x, y)|^{2}$ can be recorded on a photosensitive medium. These mediums usually change their transmission proportionally with the exposure intensity $I$ and the exposure time $\tau$ of light over them. We can define the energy density of light, as the product of these two variables: $E=I \tau$

After being exposed, we will have a plaque with a transmission:

$$
\begin{gathered}
t(x, y)=t_{0}+\beta \tau I(x, y) \\
t(x, y)=t_{0}+\beta \tau r^{2}(x, y)+\beta \tau o^{2}(x, y)+\beta \tau r o(x, y) e^{-i 2 \pi \sigma_{r} x} e^{-i \Phi(x, y)}+\beta \tau r o(x, y) e^{i 2 \pi \sigma_{r} x} e^{i \Phi(x, y)}
\end{gathered}
$$

For the reconstruction process, of the object wave, a reference wave $\mathbf{r}=r e^{-i 2 \pi \sigma_{r} x}$ is used. We illuminate the hologram with the reference beam to obtain

$$
\begin{aligned}
& \mathbf{u}(x, y)=\mathbf{r}(x, y) t(x, y) \\
& \mathbf{u}(x, y)=t(x, y)=\left(t_{0}+\beta \tau r^{2}+\beta \tau o^{2}\right) \mathbf{r}(x, y) \\
& +\beta \tau r^{2} \mathbf{o}(x, y) \\
& +\beta \tau r^{2} \mathbf{o}^{*}(x, y) e^{i 4 \pi \sigma_{r} x}
\end{aligned}
$$

We can identify four parts from the last expression. The first part is the same reference beam and with a small modulation given by $o^{2}$ and represents the order zero.

The second term is the object wave and the third term is the conjugate complex of e object wave that corresponds to the $1^{\text {st }}$ and $-1^{\text {st }}$ diffraction order respectively.

The most important part of this result is the second part, which is an exact copy of the object beam, and to an observer, it will look indistinguishable from the original.

Until this point, the basic principle in which holography is based was presented. We must remember that all of this principles work for coherent monochrome light beams and breaking any temporal or 
spatial coherence between them will result in an average of phases, and the only result we will obtain is a blur.

When holography started making great results, newsmen were invited to see them and surprised, but all they were seen could only be obtained under the strict conditions of the optical lab illumination.

Next, we will see two different applications of this principles that are particularly well suited for display applications, because both of them has results that can be seen outside an optics laboratory and with natural illumination.

\subsection{Hologram techniques particularly well suited for display applications}

Discussed below are two techniques that can be reconstructed with natural illumination, even though the reason for this is different on each case.

\subsubsection{Rainbow holograms}

Rainbow holograms are a type of transmission holograms that can be reconstructed with white light. They were invented by Dr. Stephen A. Benton in 1968. The process to record a rainbow hologram consists in two steps. The first step involves creating a traditional transmission hologram. In the second step, an image is reconstructed from the first hologram, but the illumination must come from the other side, to create a real pseudoscopic image. A horizontal slit is inserted in the light path. The new hologram will then contain information from both the image and the slit.

When this second hologram is reconstructed with white light, the reconstructed object will be seen through the slit, which will diffract the light, making each color reconstruct the object at a different angle, creating a series of slits in the entire visible spectrum.

Because all the colors reconstruct the same vertical view of the object, the vertical parallax is lost, which usually doesn't matter, as we are used to see an object only from certain vertical height. Therefore this kind of holograms is called horizontal parallax only holograms (HPO).

\subsubsection{Color rainbow}

To obtain color in rainbow holography, three holograms are chosen, each one with the information corresponding to red, green and blue respectively. They have to be arranged so that the angle in which the 
red information hologram reproduces red color corresponds to the angle in which the green information hologram reproduces green color, and the blue information hologram reproduces blue color.

If these three holograms are recorded together with the process of rainbow holography, when a white light reconstruction takes place, in a certain angle, the information of the three color channels will correspond to the real colors of the objects. At any other angle, different colors will appear.

When this is done from a real life model, the model has to be repainted in a way that simulates that a red, green or blue color filter is present, so the color information can be encoded.

\subsubsection{Lipmann color holograms}

Reflection type holograms were invented by Y.N. Denisyuk in the URSS. The difference with Fresnel holograms is that the reference beam and the object beam converge on the hologram plane coming one from the front and the other from the back. The advantage of this is that the reconstruction can be done with white light.

In 1962, Denisyuk presented this scheme, conceptually similar to the first color photographic process invented in history, by Gabriel Lippmann in 1891. The coherent object wave is reflected on the subject, which is located after the holographic plate, and is propagated backwards, superposing the incoming wave. Both waves form a tridimensional distribution of stationary waves. This distribution is recorded by the photoresist material in all the thickness. These holograms are known as thick holograms, or volume holograms. The result will be a tridimensional grating, modulated, periodic, of phase or amplitude objects, that represents data.

When reconstructed, the incident wave will behave according to the Bragg law. An important feature of volume holograms is its interference of the wavelength with the reconstruction. Only a certain wavelength will be reconstructed, according to Bragg law

$$
2 d \sin \frac{\theta}{2}=\lambda
$$

Where $\theta$ is the angle of the reference wave during recording, and $d$ is the distance of the lattice planes.

Another great behavior of volume holograms is that the same volume can fit several holograms that can be reconstructed independently, if they are recorded with different angle or different wavelength. 
G.W. Stroke and A.E. Labeyrie went a step further and combined multiple volume holograms to create what is known as white light reflection holography. Here, the reconstruction beam is a white light beam, with a wavefront similar to the one used to record the hologram. When it is illuminated, only the specific wavelength, penetrating the grating with the appropriate Bragg angle will form a tridimensional virtual image. If a hologram recorded with a red laser overlaps one recorded with a green laser, and the same for a blue laser, the white light will reconstruct the three holograms at the same time, creating a full color feeling.

This type of holograms keeps a similar procedure with the color photography method by interference layers invented in 1891 by Gabriel Lippmann, and which made him win a Nobel Prize in 1908.

\subsection{Ideal stereogram resolution for the human viewer}

For a human being, a hologram should be sampled in a way that the width of a pixel $w_{h}$ doesn't exceed

$$
w_{h}=D \tan \left(\frac{1}{60} \operatorname{deg}\right)
$$

with an observer looking at the hologram from a distance $D$. These will guarantee an ideal resolution and a continuous feeling along the hologram, given a human visual acuity of around 1 arcmin (3). In order to fulfill the Nyquist limit, a sampling of twice the highest spatial frequency should be used when capturing the scenes to be used on the holograms.

Assuming a distance of $35 \mathrm{~cm}$, the width of each hogel shouldn't be bigger than

$$
w_{h}=350 m m \tan \left(\frac{1}{60} \operatorname{deg}\right)=0.101 m m
$$

\subsection{Advanced techniques}

Holography didn't stall with the first wave of inventions. Moreover, it benefited itself from the computer advances, taking digital encoding for itself. Holography mixes well with digital representation, as a simple transmission hologram can be seen as a two dimensional matrix of intensity values, which is the ideal representation for digital conversion.

But having the digital representation of a hologram printed has its challenges. These challenges are very similar to the ones present in the imprint process of electronic devices, furthermore the size of the electronic details printed in electronics and the fringes in the holograms are more or less the same, and 
this means that technology developed for this application can be implemented or adapted to record holograms. Two techniques mentioned for comparison purposes are the Dot-Matrix technique and the Ebeam technique, the comparison with these two technologies comes in hand, as these two are currently used in the industry for the process we are interested in.

Not only the hologram recording process was benefited from the digital world, and the encoding of the holographic information has been touched by them.

It is possible, by digital means, to select if some part of the calculated interference pattern is going to be recorded or not, and this way, for example, eliminate the zero order on the reconstruction, like in kinoforms, or build optical elements just selecting the etching depth point by point.

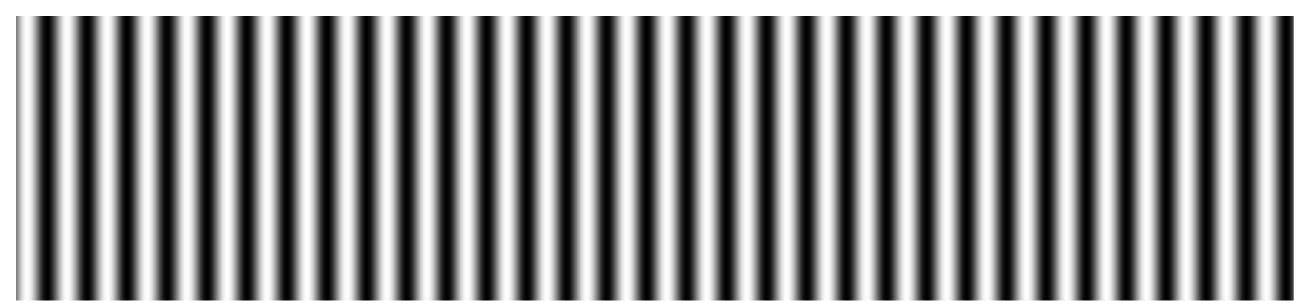

Fig. 1.1 Digital Representation of the interference pattern of two wave planes

\subsubsection{Dot-matrix}

This technique gives as a result a matrix of diffraction gratings in the shape of small dots recorded over a photosensitive resin plate to form a relief surface. Each of the dots has a calculated period and orientation that will form a full pattern when the whole matrix is considered. Due to the properties of a relief grating, a high efficiency and good visual angle can be obtained, making this kind of printing a good candidate when choosing something with visual impact for display or packing. A boom of this kind of holograms appeared in the 90s, when a lot of works about the subject surfaced (4) (5) (6). As other kinds of digitally calculated holograms, this kind of holograms have also improved as a result of better electronics ( precision positioning XY stages ) and computers over the years.

Dot-matrix holograms have been popular for decorative display and security for some time. With their resolution of up to 10 micrometers, they are very difficult to copy, and used on security continually, given the flexibility they have as computer designed holograms. 
As mentioned before, the recording is based in small diffraction gratings known as holopixels, recorded with the creation of an interference pattern between two planar waves over the photoresist. If one of the beams varies the angle and position when recording, different grating frequencies and orientations can be obtained. The holopixels are arranged in a 2D array that will form the hologram. When reconstructed with white light, each holopixel will redirect the light to a certain angle and disperse the different colors. The whole matrix of holopixels will form the desired figures and effects.

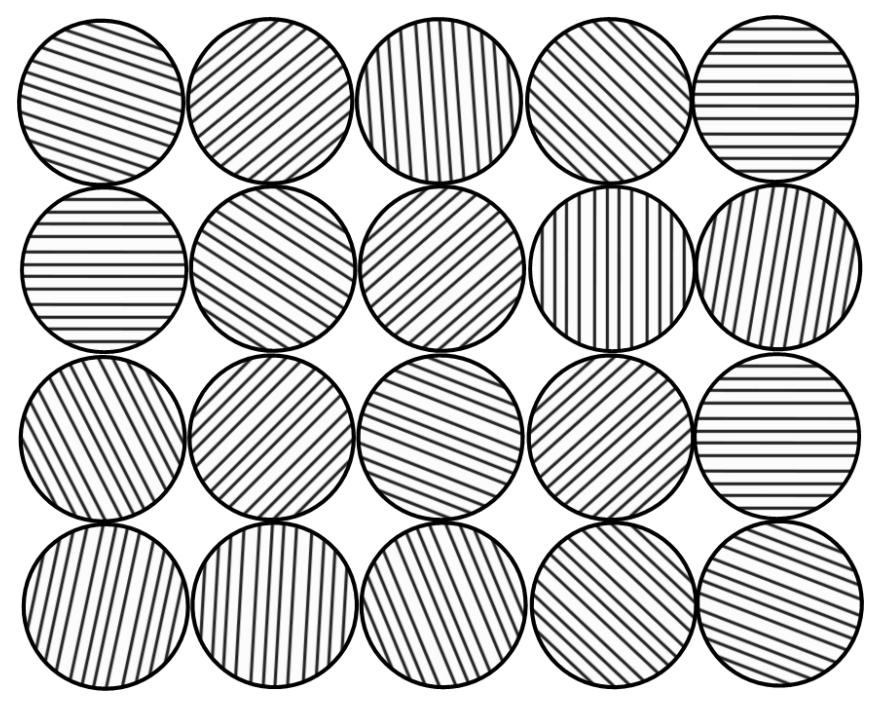

Fig. 1.2. Representation of a Dot-Matrix

Each diffraction pixel contains a plane wave diffraction grating, consisting on straight, parallel lines.

A look-up table is used to determine each holopixel orientation and spatial frequency, so the complete hologram can be generated from a single bitmap image.

From equation 1.12, we can obtain the interference pattern of two coherent plane waves moving in space (7) as 


$$
I(x, y)=I_{1}+I_{2}+2 \sqrt{I_{1} I_{2}} \cos 2 \pi\left(f_{x} x+f_{y} y\right)
$$

Where $f_{x}$ and $f_{y}$ come from the representation of the planar waves expressed as the expansion of the spatial term on equation 1.13 as:

$$
E_{i}(r)=A_{i} e^{i \frac{2 \pi}{\lambda} k_{i} \cdot r}
$$

Where the wave vector $k$ can be expressed with its direction $\operatorname{cosines}$ as $k_{i}=<\cos \alpha_{i}, \cos \beta_{i}$, $\cos \gamma_{i}>$ for both waves interfering $E_{1}$ and $E_{2}$.

From this representation we can obtain two values, $d$ and $\phi$, that will represent the two parameters we can control from each holopixel: the spatial frequency $1 / d$ and the orientation $\phi$. These two parameters will be controlled only varying the angle of one of the interference waves, respect to the other beam $(1 / d)$ and respect to the hologram orientation $(\phi)$.

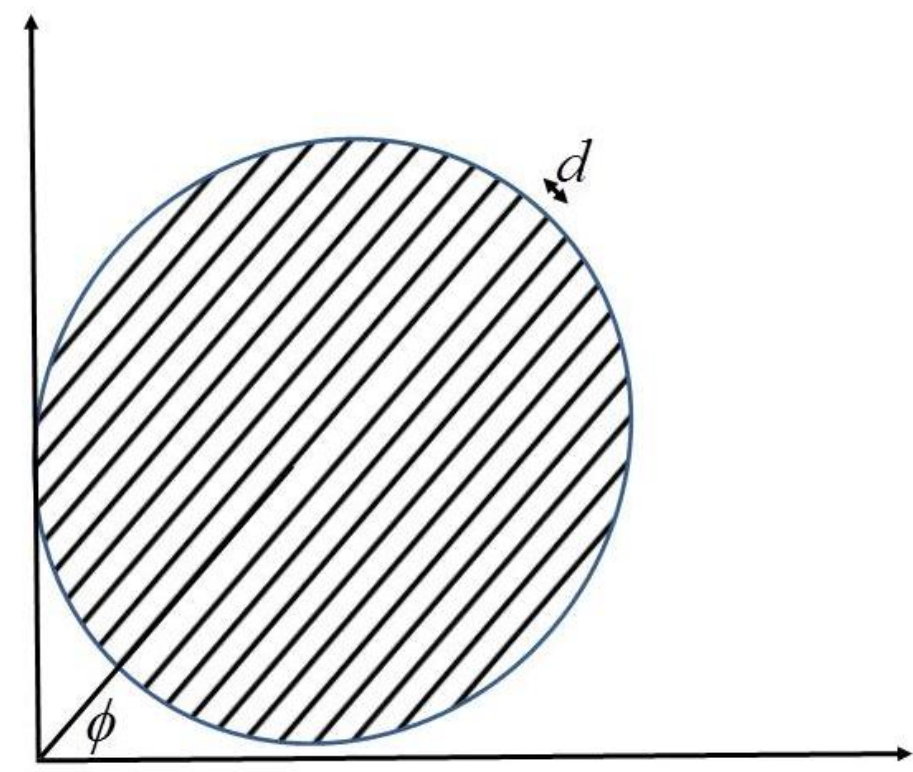

Fig. 1.3. Parameters affecting a holopixel in Dot Matrix hologram printing 
Because is a fast way of obtaining a hologram, some techniques have been implemented to try and improve the efficiency and overall quality of the results, like trying to cover the maximum percentage of area over the resist with the dots, so instead of circular dots, more squared ones are desired. But even with these improvements, the size of the dots will be always a natural limit of the technique, because the image resolution will have this basic dot size as its limit.

\subsubsection{E-beam}

E-beam holography consists on using e-beam lithography to record a hologram. One beam on electrons is shot into a coated substrate to etch a substrate or induce deposition of substances into it. Due to its high precision, it allows the correct recording of a hologram.

A modified transmission electron microscope can be used for this process, with an emission gun adapted.

Even though the process is easy to understand, the parts required are complex and hard to get.

The basic part is an electron gun able to fire electron in a chosen direction. First, an emitter is heated to excite electrons. These electrons will move to an anode when a high voltage is applied. Some common emitters include lanthanum hexaboride crystal and zirconium oxide coated tungsten.

A series of lenses and electromagnetic fields produced usually by to parallel plates that can be electrostatically charged will focus the electrons in a directed beam. This is called the electron optical column.

The resulting beam can be focused on the resist surface, where the high energy charged electrons will break the material bonds for the further etching or deposition.

The beam can be used to scan an entire area, etching different places with different energy, by varying the voltage of the machine, this way, any pattern can be directed with an incredibly high resolution. 


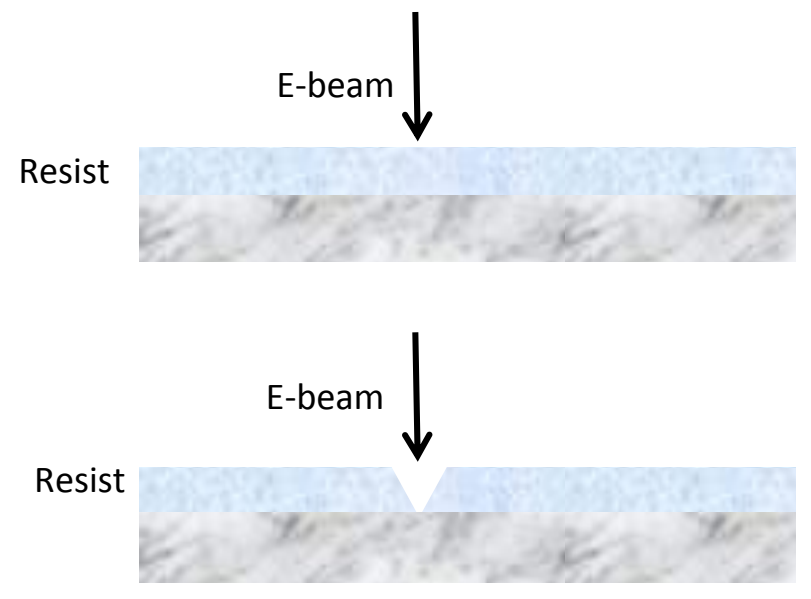

Fig. 1.4. Depth etching of a photoresist using an E-Beam

Although e-beam has this extreme accuracy, the equipment is complex and the time spent on the scan is high, this makes this process unpractical for mass production and not easily acquired for research or commercial purposes.

However, this high precision comes to a cost, and in this case, the cost is the time spent in recording, which rockets the cost of using such machine. This machine is very sensitive to noise if dirt is present, because the electrons are electric particles that can interact with matter present. It should operate in vacuum, so the costs are again increased, and complicates the process and the equipment needed.

\subsubsection{Resolution capability}

Probably the highest resolution machine for imprint lithography, an E-beam machine is capable of obtaining a resolution close to $10 \mathrm{~nm}$ and is limited mostly by scattering and system aberration in the optical side, and molecular structure of the material on the etching side. Obviously this resolution is more than enough for visual holography applications. 


\subsubsection{Kinoform}

A Kinoform is a computer generated hologram that encodes a mathematically calculated hologram pattern without using the amplitude part in the wave representation, and leaving all the encoded information on the phase (8). The recorded hologram in this case is completely transparent and the phase is the one that modulates the result.

In this case the transmittance of the recorded hologram can be expressed as:

$$
\mathbf{t}(x, y)=e^{i \Phi(x, y)}
$$

Where the phase can vary from 0 to $2 \pi$. A grayscale is used over the computer to record this phase, where different values will correspond to a different final depth of the material. The material is afterwards bleached until the burned part is removed, leaving a thickness modulation. This kind of effect can only be calculated with the help of a computer, because the calculated hologram should not contain any information to reconstruct the zero or the $-1^{\text {st }}$ order that includes the conjugated information of the hologram.

A major advantage of a Kinoform is that theoretically, all the diffracted light will form the image, without the presence of another virtual image or higher order images that exist in other kinds of holograms and with whom the energy is distributed. This also removes the superimposition of the real and virtual images, making a clearer result.

Because no light is absorbed in a phase only hologram being it completely transparent, the brightness of the reconstruction is further enhanced.

Some noise is introduced in the process (9), by the assumption that only the phase can be used for the total reconstruction, but the results have shown that the noise doesn't make the technique useless, and the unique results it gives eliminating the zero order, make it a worth technique to include in the repertoire of digital holograms. 


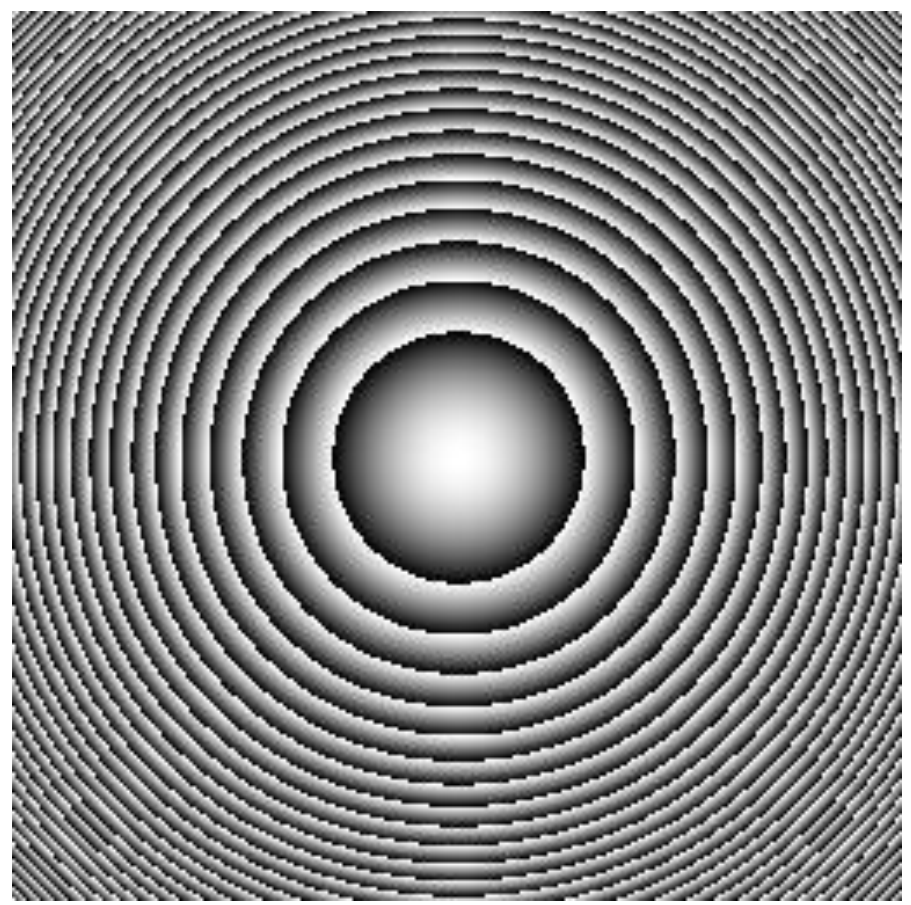

Fig. 1.5. Kinoform Lens

\subsubsection{Diffusers}

A diffuser ordinary generally consists of a surface scattering light in all directions. This is usually the case of uneven surfaces on a wavelength scale, like a plastic surface or a frosted glass. A perfect diffuser should be isotropic, or direction independent. The variation of the reflective brightness with direction is dependent of the surface smoothness.

A transmission hologram of two plane wave can act as a diffuser. 


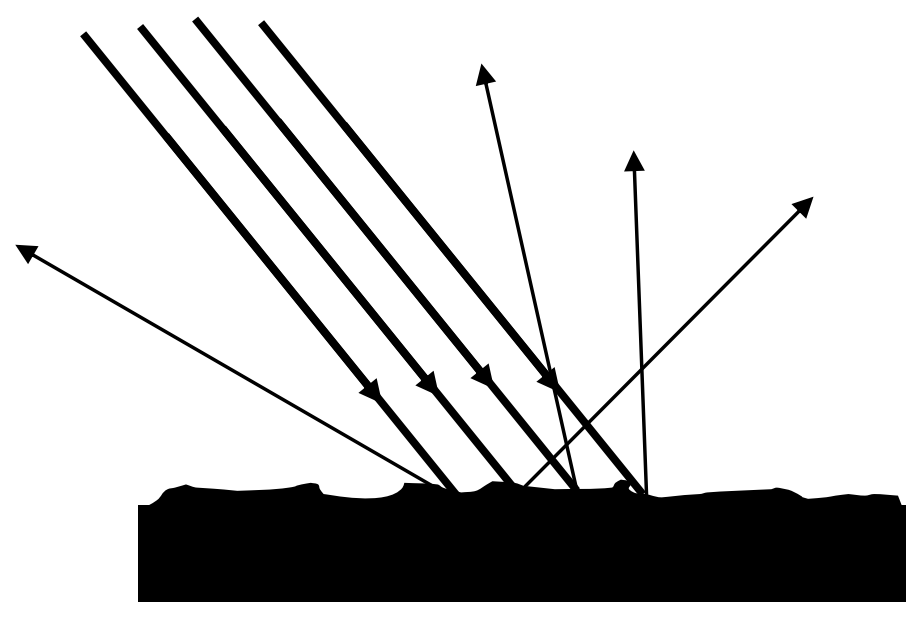

Fig. 1.6. Diffuse reflection

Given their properties, diffusers are used in optics when a homogenous wide illumination is needed. It is especially useful when doing holography, when the objects to be recorded are big and the light brightness should be uniform.

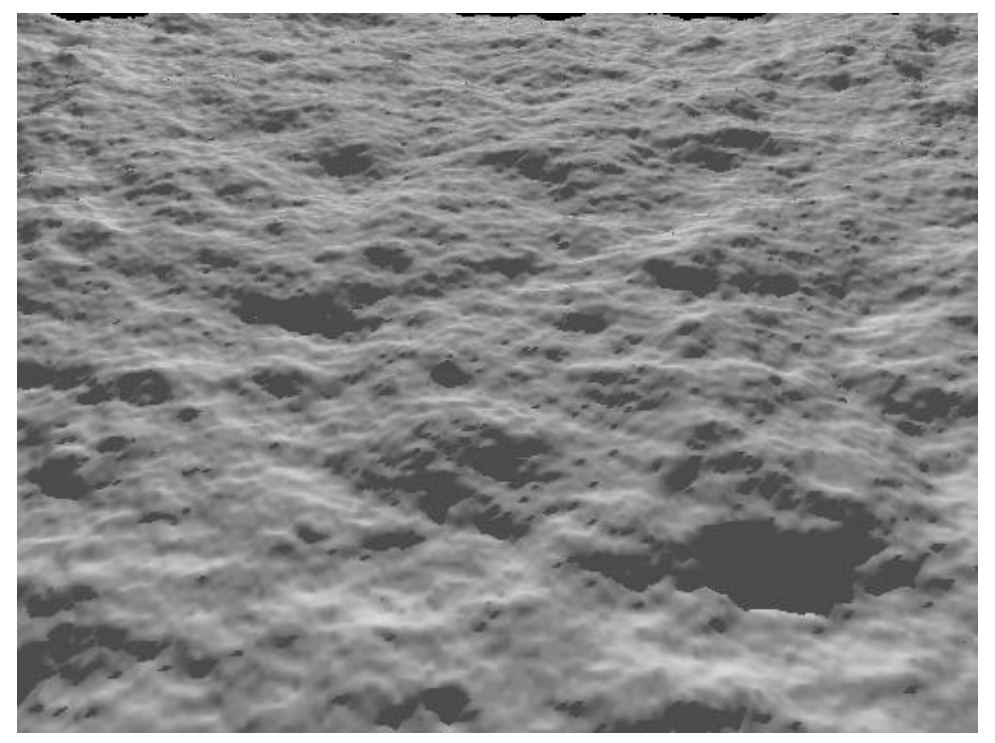

Fig. 1.7. Diffuser surface

\subsubsection{Microtext}

In a pattern consisting on diminutive characters is recorded using photolithographic means, if the characters have the adequate size, they will serve as light diffusers and even as a diffraction grating. If a text or a logo with a lot of details is recorded, due to the randomness of line directions and patterns 
present with the combination of different letters and characters, the resulting surface after the recording will produce the effects of a really well made diffuser.

Even more, if the characters are not randomly aligned, but hidden inside designed grids or gratings, a hologram can be built around them without introducing a lot of noise to the whole result.

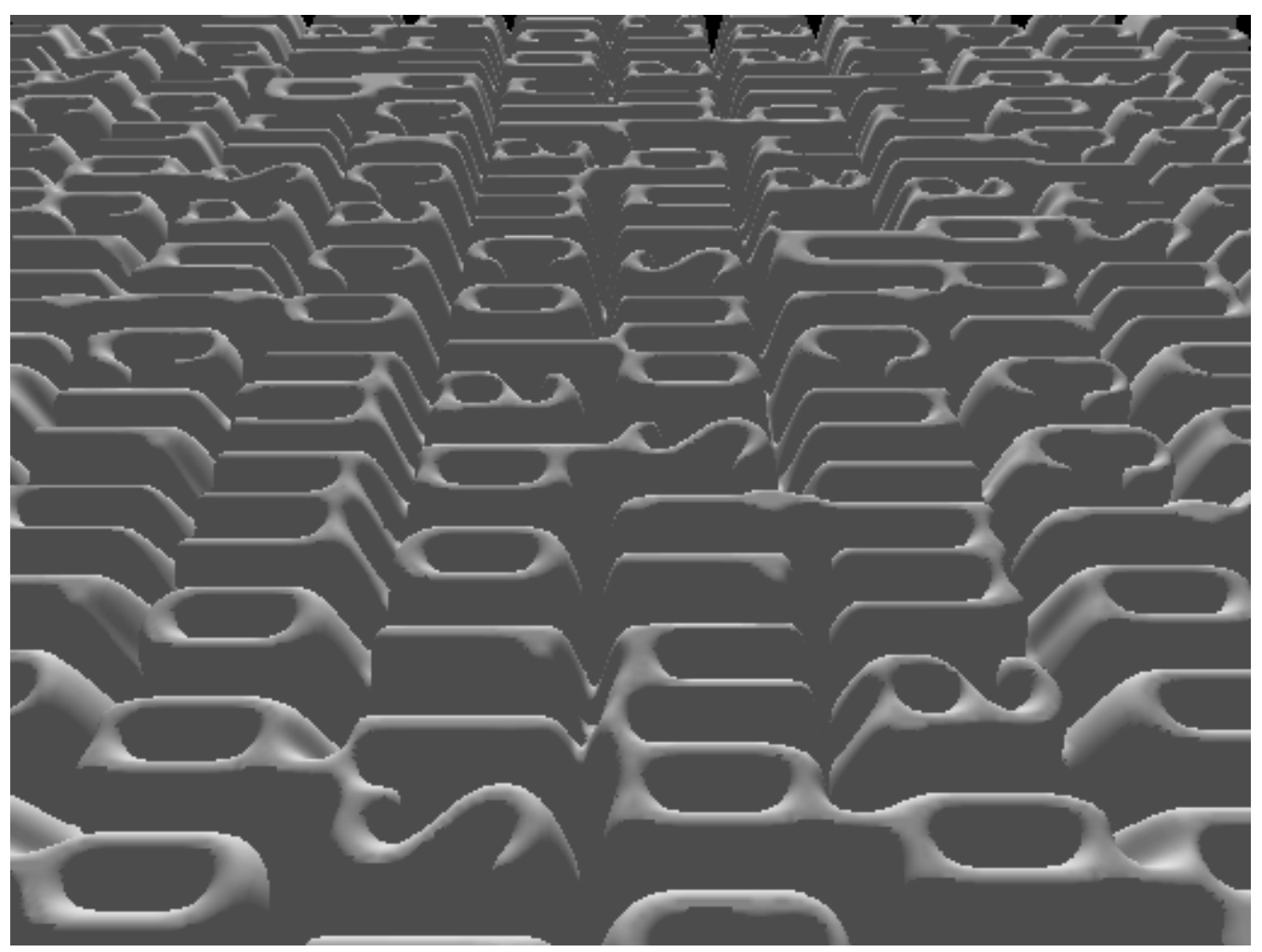

Fig. 1.8. The Microtext can work as a diffuser scattering the light in all directions 


\section{The basic concept}

This work is based on the basic digital 2D/3D digital hologram recording optical set up shown in Fig. 2.1 that was proposed and practically built by Dr. DerKuan Kang in 2001. It is capable of recording relief type optical constructions with E-Beam-equivalent resolution.

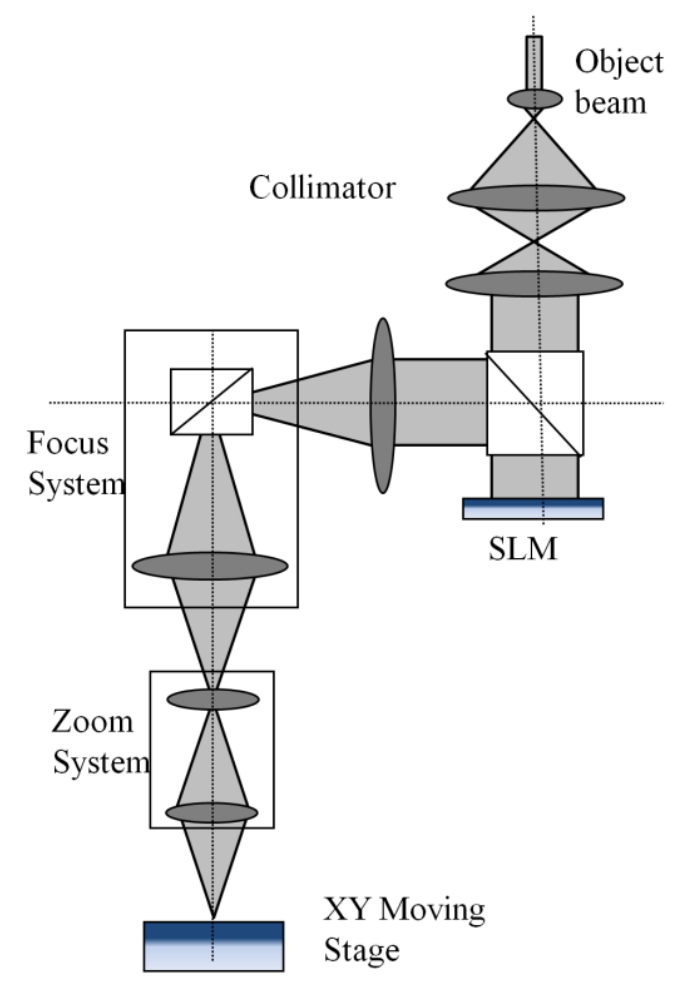

Fig. 2.1. Basic digital thin hologram recording diagram

In this optical setup, a laser beam is applied through a collimator and a beam splitter to illuminate an amplitude type SLM (Spatial Light Modulator) where a calculated optical data/ pattern is displayed. A high speed response autofocus system is applied to control the SLM image conjugated into a photosensitive material surface located on the top of an XY stage. A zoom lens set up is designed to adjust the correct image magnification. In the process of moving the XY stage from one exposure spot to the next, the conjugated image point can be lost due to the roughness of the material surface or/and the vibration of the entire system. Therefore, a micro-order response auto-focus system is essential and necessary. 
A DLP (Digital Light Projector, Texas Instruments) is used in the system as the SLM which has $1024 \times 768$ pixels and about $13 \mu m$ pixel size.

It is necessary, however, to avoid the noise that appears in the projected image due to the laser beam scattered by dust through the optical path. The noise causes each exposed image blur and reduce the diffraction efficiency in the final hologram. In order to minimize the noise, a second SLM was inserted. This SLM was a phase type LCD panel (Displaytech Inc., USA) with $1024 \times 768$ pixel and about $15 \mu \mathrm{m}$ pixel size. In effect, given that no actual interference is taking place in the recording, it is not a requirement to use a laser beam as the source. Instead of a laser beam, an incoherent light source can be introduced, where the wavelength corresponds to the sensitivity of the material. This alternative light source can be useful to avoid speckle noise. The introduction of the second SLM is also useful when the Lippmann recording mode further described on this thesis is introduced.

\subsection{Real set up}

As shown in Fig. 2.2 below, the laser beam illuminates the phase type SLM, marked as SLM2, and then is reflected onto the second amplitude type SLM, marked as SLM1. A random phase pattern is generated every few $\mu s$ on SLM2 while an amplitude type calculated fringe/grating/data is displayed

over SLM1. Some optical elements adjusted with great precision are necessary to match the conjugating pixel locations.

On the other hand, a three color laser chamber is set and aligned carefully to obtain a single laser beam path with the capability of switching between different recording modes. A switchable on/off reference beam is oriented to hit on the image spot from the opposite side of the plaque for Lippmann type recording mode. 


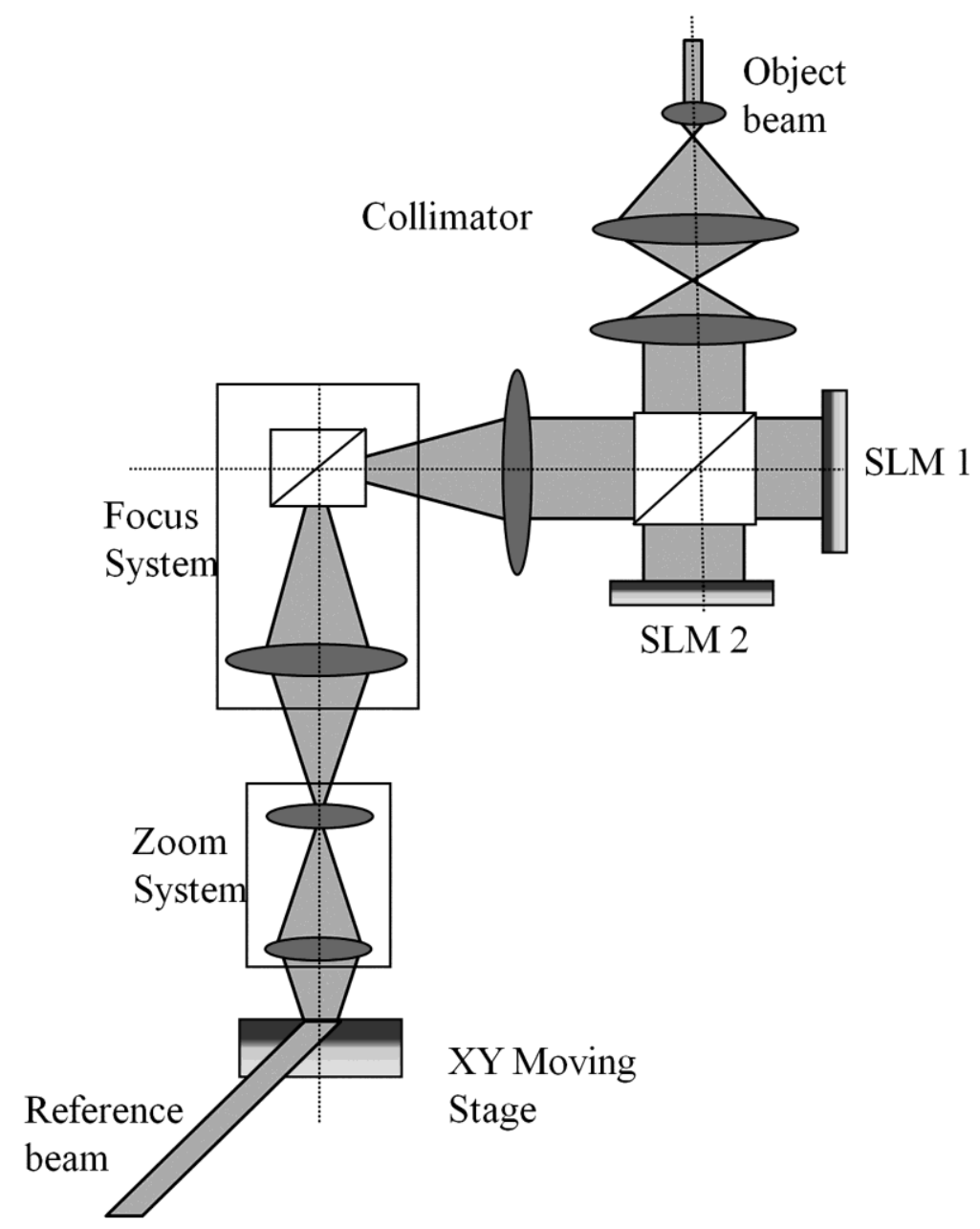

Fig. 2.2. Diagram that shows the parts used on the recording of a digital volume hologram

When this optical setup is practically used, calculated optical data is displayed on the SLM1 and further reduced in size with approximately $50 \times$ reduction, then imaged over the recording material. The photosensitive plate is transported using an XY stage with sub-micrometer order control repeatability. The entire imaging lens setup is well-designed to move up and down together with an SLM panel through a real time response auto-focusing unit. This unit will maintain the conjugation point of the input image plane over the photosensitive material plane with sub-micron order accuracy.

A laser beam is collimated and propagated into a random phase generator that eliminates the coherent speckle noise produced from dust and imperfections in the optics. The random phase generator used is the previously presented phase-controlled modulator from Displaytech Inc. (SLM2) with the pixel pitch of $15 \mu \mathrm{m}$ (Fig. 2.2). SLM2 is also used as a specified phase generator when is necessary to digi- 
tally add phase information on the final optical output. The wavefront after SLM2 is further reflected into a second SLM, the amplitude controlled SLM1, with a pixel pitch of $13 \mu \mathrm{m}$ where the calculated amplitude image is displayed and projected onto the photo-sensitive material surface. In principle, the full image displayed in SLM2 and SLM1 with a total of $1024 \times 768$ pixels, could be reduced to a size of $200 \mu \mathrm{m} \times 150 \mu \mathrm{m}$ and projected as an exposed element on the material surface. However, practically only an area of $256 \times 256$ pixel is used given a limitation in the aperture design on the final optical system.

The optical recording resolution of the optical setup is based on the Numerical Aperture (NA) given by equation ( ):

2.1

$$
N A=\sin \theta=\frac{D}{2 f}
$$

where, $\theta$ is the angle, $D$ is the diameter of the entrance pupil and $f$ is the focal length.

With a resulted $N A$ of 0.45 , we can achieve about 0.4 micron meter resolution. For a plain grating recording, this means one line-pair can be achieved in 0.8 micron meter. This will achieve up to 1200 line-pair/mm.

As mentioned above, in principle, each exposed cell has a size up to 200 micron containing $1024 \times 768$ pixels of digitized data and practically $256 \times 256$ are used. It is reasonable to divide it into a sub-cellmatrix, using programming techniques to achieve higher image resolution until the smallest sub-cell reaches one line-pair. In this case, the highest image resolution for a plane grating is about $2.5 \times 10^{4} \mathrm{dpi}$, and 1200 line-pair presents 40 degree off-axis hologram. For a color 3D image using plain grating technique (10) (11), the image resolution is limited to about 1500 dpi since the area is divided to display the R,G and B color components and perspective views. To preserve this high resolution, an autofocus system is also present as part of the general layout. This part of the system will allow maintaining every image on focus, avoiding the errors that could be present because of the moving platform. 


\subsection{Changing modes}

Another thing to consider is the flexibility of operation between different recording modes. It cannot be considered a single mechanism if the changes between one mode and another oblige the user to disarm and rearrange the system every time a different mode is needed.

Ideally, all modes should coexist at the same time, but given the differences in the resolution and optical paths, a few physical changes must be made. If these changes can be reduced to translation of optical components and shutting on and off some beams, then it should be possible to automate the process.

As will be discussed in the next chapters, a setting similar to the one described in the latter paragraph can be implemented and the results of the physical construction of the system will be analyzed. 


\section{Digital relief type (Rainbow) holograms process}

This chapter will describe the relief type (rainbow) hologram recording process.

\subsubsection{Recording Material}

In order to duplicate holographic optical elements (HOE), a relief type photo-sensitive material called photoresist is used in this research for rainbow type hologram recording mode. The thin photoresist material will react according to the intensity distribution of the light source. After the developing process, the reacted material will be removed corresponding to the exposed pattern distributions; remaining only a depth distribution proportional to the light source distribution.

\subsubsection{New white light 3D CGHS proposal}

CGH (Computer Generated Holograms) can model arbitrarily complex wavefronts, like non-spherical wavefronts, which are extremely difficult to obtain with regular optical elements. They can be generated by simulating different mathematical optical models and virtual optical setups. The use of computer modelers circumvents the problems of complicated setups used in holography but introduces other set of problems, like limited resolution. Furthermore, the huge quantity of data takes its toll in time calculation (12). Some ideas had been proposed for resolving the calculation problems. For example, decrease resolution in the recording process. Alternatively, the idea of using e-beam systems and laserwriting methods emerged in recent years for simplifying the recording process (10). It is reported that a micron resolution can be achieved using these techniques. On the e-beam writing system case, however, the cost of the equipment and the recording time limits its actual application (11).

It is the intention of this work to apply the optical setup shown in Fig. 2.2 to write CGH with relatively big dimensions as described above. In order to recognize the reconstructed wavefront visually, a special $\mathrm{CGH}$ is proposed. This proposed CGH introduces the process to generate color holograms (13). The setup uses an incoherent light wavefront as a source and a digital light device (DLP) to modulate it. The CGH phase data, formatted into amplitude images is then projected through the micro-imaging system onto photo-resist material. The full system provides a 0.4 micron resolution and about 100 times a speed gain compared to an e-beam in high speed pattern recording in similar conditions. 


\subsubsection{Mathematic model}

In the calculation of the $\mathrm{CGH}$, the mathematical model described here is applied. There is a series of perspective images introduced on the input plane (left side of Fig. 3.1), that are then focused simultaneously on the hologram (right end) plane through an imaging lens (middle) where a multi-aperture shutter is set. Notice the impossibility of building this setup in real life, because the simultaneity of focusing the full series of images over the hologram plane.

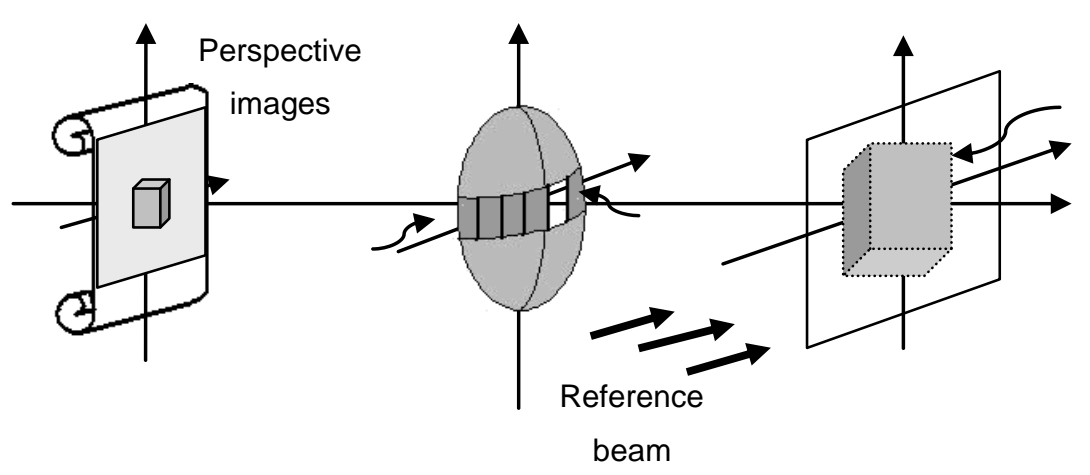

Fig. 3.1. The optical setup of the CGH calculation

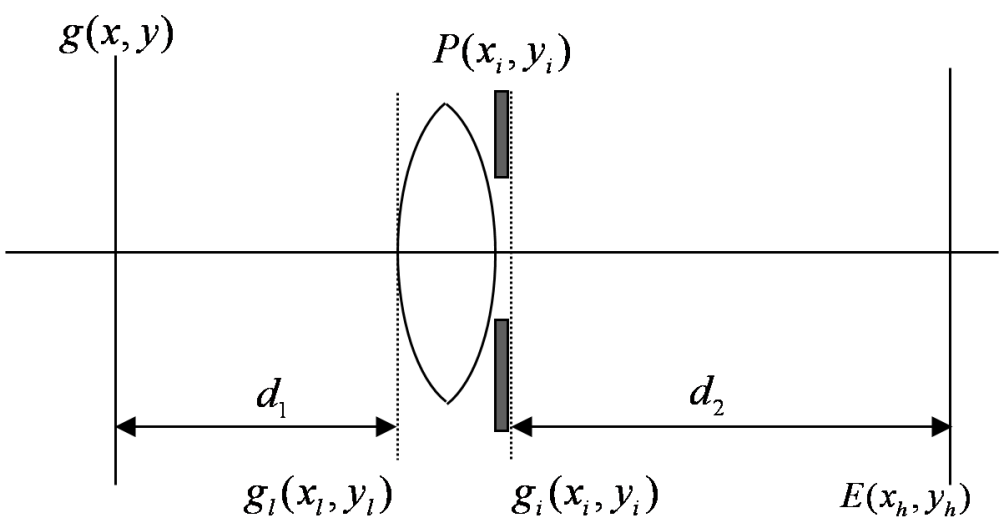

Fig. 3.2. Mathematical model of the optical setup

The intermediate values of the wavefront in the setup can be represented as: 


$$
g_{i}\left(x_{i}, y_{i}\right)=\frac{1}{j \lambda d_{1}} e^{j k d_{1}+\frac{x_{i}^{2}+y_{i}^{2}}{2 d_{1}}} F\left\{g(x, y) e^{j k \frac{x_{i}^{2}+y_{i}^{2}}{2 d_{1}}}\right\}
$$

$$
\begin{aligned}
& g_{i}\left(x_{i}, y_{i}\right)=g_{i}\left(x_{i}, y_{i}\right) e^{j k \frac{x_{i}^{2}+y_{i}^{2}}{2 f}} P\left(x_{i}, y_{i}\right) \\
& \therefore E\left(x_{h}, y_{h}\right)=\frac{1}{j \lambda d_{2}} e^{j k d_{2}+\frac{x_{i}^{2}+y_{i}^{2}}{2 d_{2}}} F\left\{g_{i}\left(x_{i}, y_{i}\right) e^{j k \frac{x_{i}^{2}+y_{i}^{2}}{2 d_{2}}}\right\}
\end{aligned}
$$

The equation 3.1, 3.2 are applied to each image. The aperture function $P\left(x_{i}, y_{i}\right)$ represents the window that is relative to each view and it is a rectangle function in two dimensions right after the imaging lens. The whole propagation of the wavefront before lens of images using Equation 3.1 and

3.2 is obtained. The final calculation of wavefront propagation from lens to hologram is done using Equation 3.3. Finally, a reference wavefront is added to get the final data. Here, the equation of the Fresnel propagation (14) is used. The hologram is set as a viewing distance about $25 \mathrm{~cm}$ and size of about $2 \mathrm{~cm} \times 2 \mathrm{~cm}$.

\subsubsection{Fourier Transform Calculation}

One of the first problems to resolve was the quantity of data for processing. One image of $1028 \times 1028$ pixels generates a hologram of $3.6 \mathrm{~mm} \times 3.6 \mathrm{~mm}$. Therefore, for generating a hologram of $1.0 \mathrm{~cm} \times 1.0 \mathrm{~cm}$ or bigger, we need have image more than $3000 \times 3000$ pixels at least. Also we have to take into consideration that we need many object views, 8 images or more. All must to be in 24 bit colour images. In the FFT calculation of about 24 images of $3000 \times 3000$ pixels using a regular computer with Pentium IV, $2.6 \mathrm{GHz}, 512 \mathrm{MHz}$ is used. We divided the data into blocks and read only one portion each time of data. As shown in Fig. 3.3, each block is recorded in separated file. We use the property of 2 dimensions FT, in which calculate the FT of rows first, and then the FT of the columns. Ap- 
plying this method, we can avoid of running out the memory of the computer. The calculating process is extremely slow due to the writing and reading time to the hard disk. Once the corresponding number of block is chosen, however, the entire calculating time can be greatly reduced.
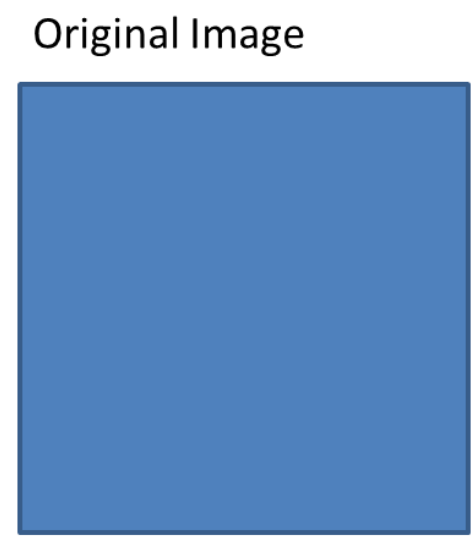

Divide in 4 parts

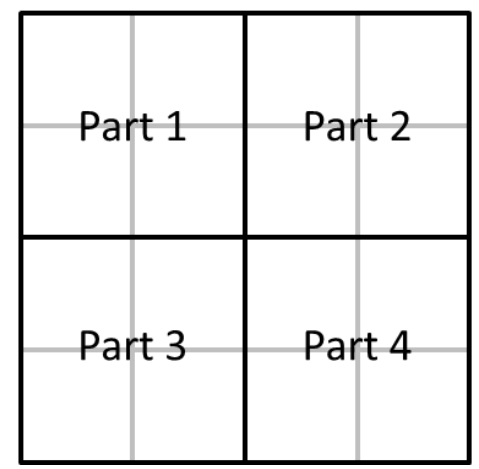

Divide in NxN blocks

\begin{tabular}{|l|l|l|l|}
\hline Block 0 & Block 1 & Block 2 & Block 3 \\
\hline Block 4 & Block 5 & Block 6 & Block 7 \\
\hline Block 8 & Block 9 & Block 10 & Block 11 \\
\hline Block 12 & Block 13 & Block 14 & Block 15 \\
\hline
\end{tabular}

Fig. 3.3. Block diagram for files in the Fourier transform calculation

In digitizing the data, Lee's CGH is applied in this experiment. The wavefront data result is divided into cell matrix array for each point of the complex amplitude. Each cell is divided in 4 parts in the vertical direction. The two first parts represent the real part of the data and the two second parts represent the imaginary part of the data. For each point, in the case of the real or imaginary part of the data is greater than zero, the first cell related will be filling with the value of the real amplitude of the point. Otherwise, the second part is filled and the initial value of whole the cell is black, as shown in Fig. 3.4. 


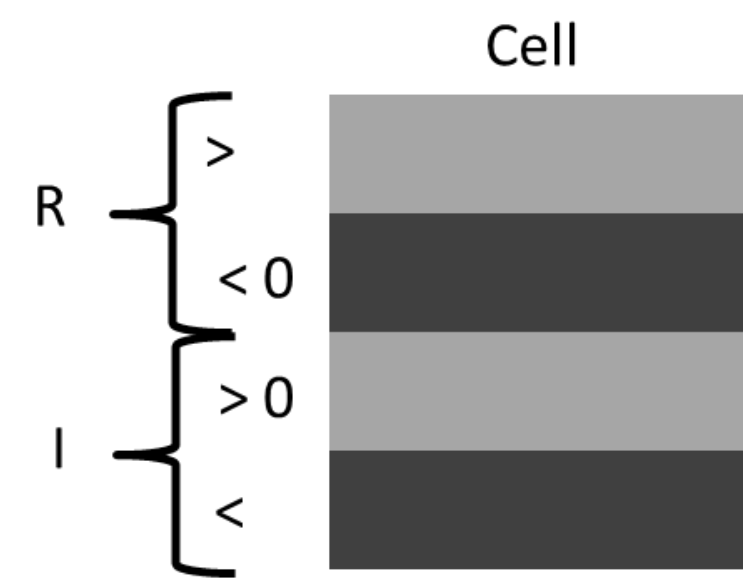

Fig. 3.4. Cell block of each data digit

Optical data and fringe patterns are calculated in advance in these so-called digital rainbow holograms. The output data is directly printed over a photo-resist through the setup shown in previous sections. The fringe patterns are composed by optimized etching depths over the photo-resist layer.

The following section shows in diagram form the process explained here, and how digital files were used to store the information of the hologram. The first diagram shows a general view of the process, meanwhile the other ones specify the functions and methods used. 


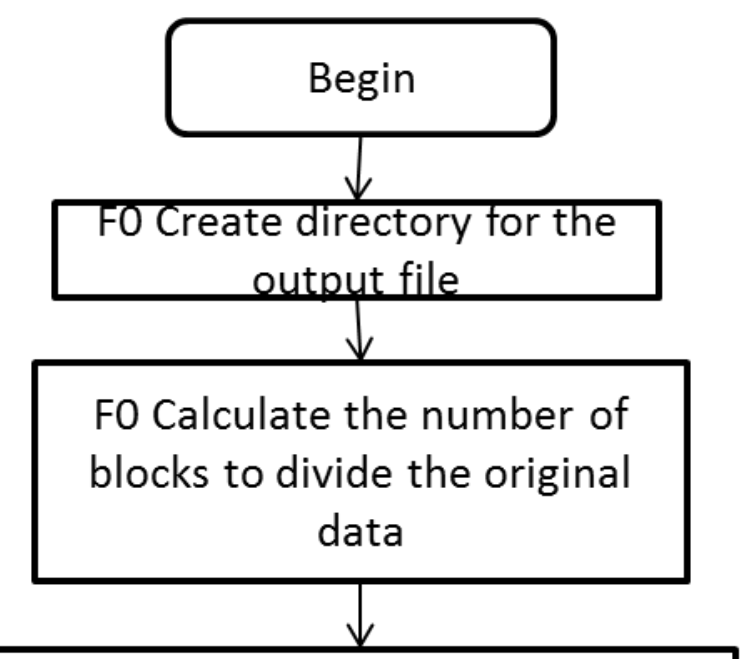

F1 Calculate the Fourier transform of each row, data file dataimag_i/r, and save like columns in the files datarow_rh

F2 Calculate the Fourier transform of each row of the files datarow_i/r and save it in the files datacol_i/r

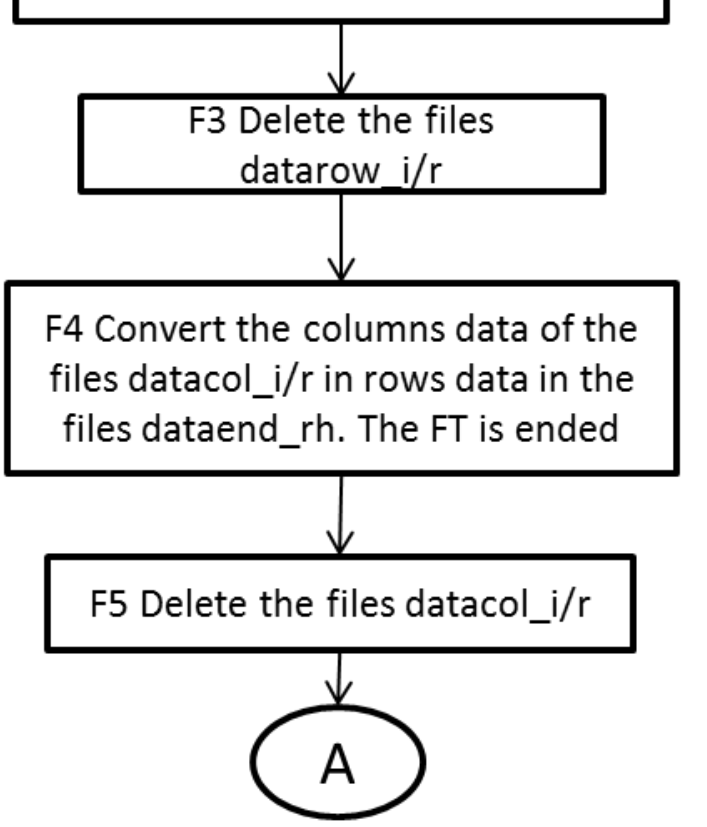

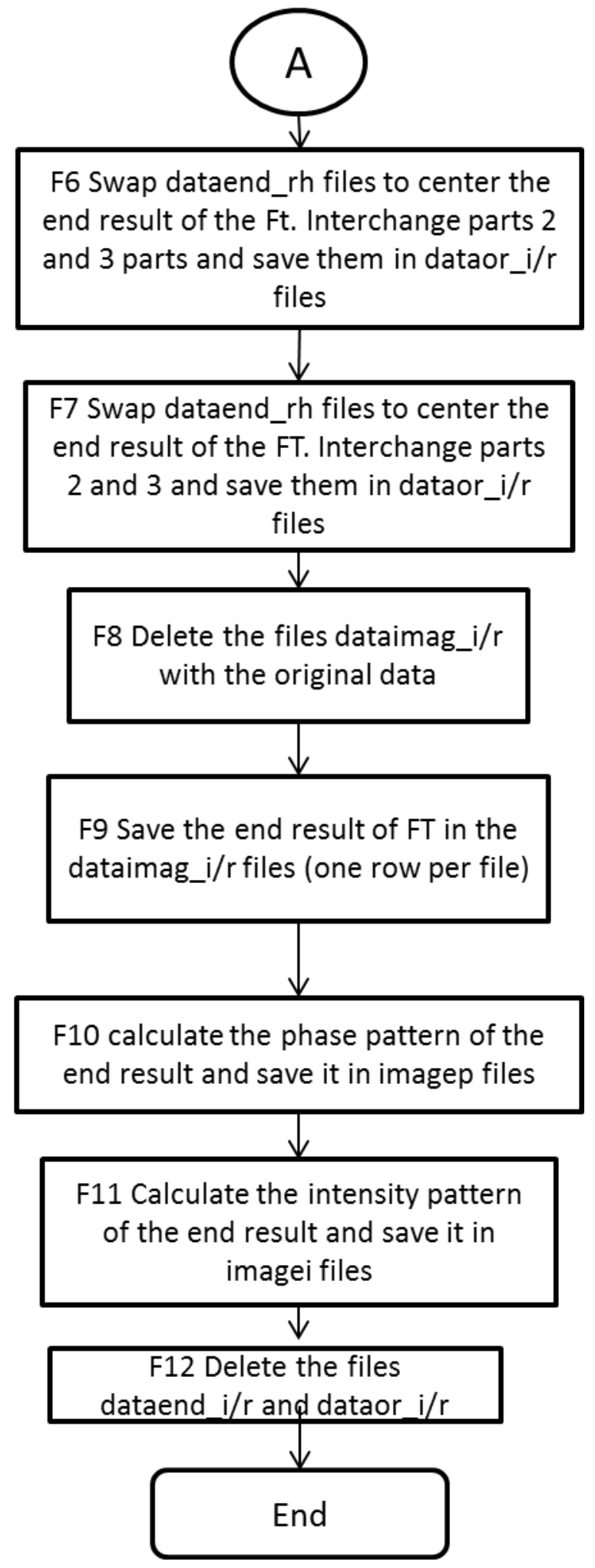




\section{F1}

The data is divided in $N \times N$ blocks of $n \times n$ size each block where $n \leq 512$. N should be multiple of 2 . This is to create small data files.

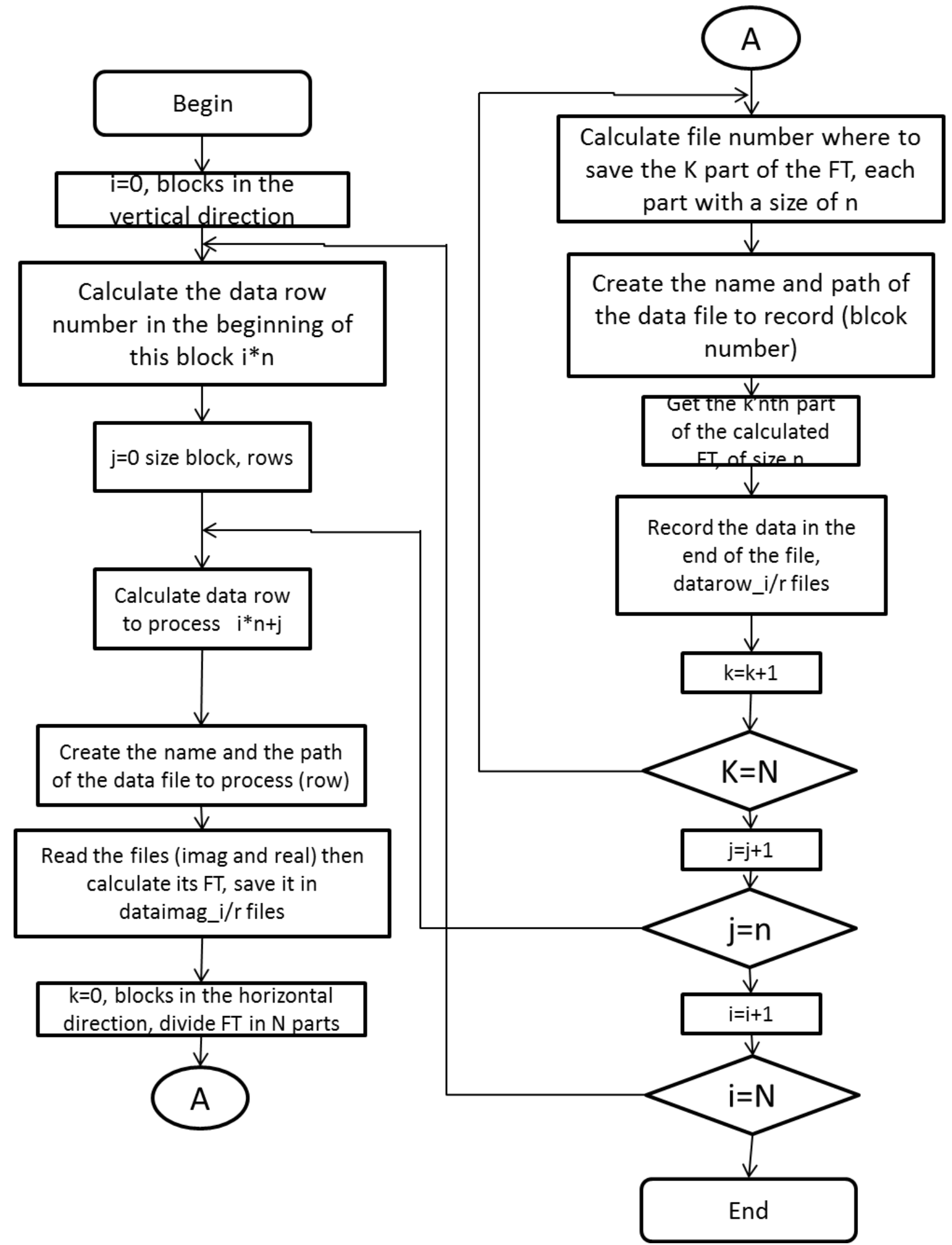




\section{F2}

The data is divided in $N \times N$ blocks of $n \times n$ size each block where $n \leq 512$. N should be multiple of 2 .

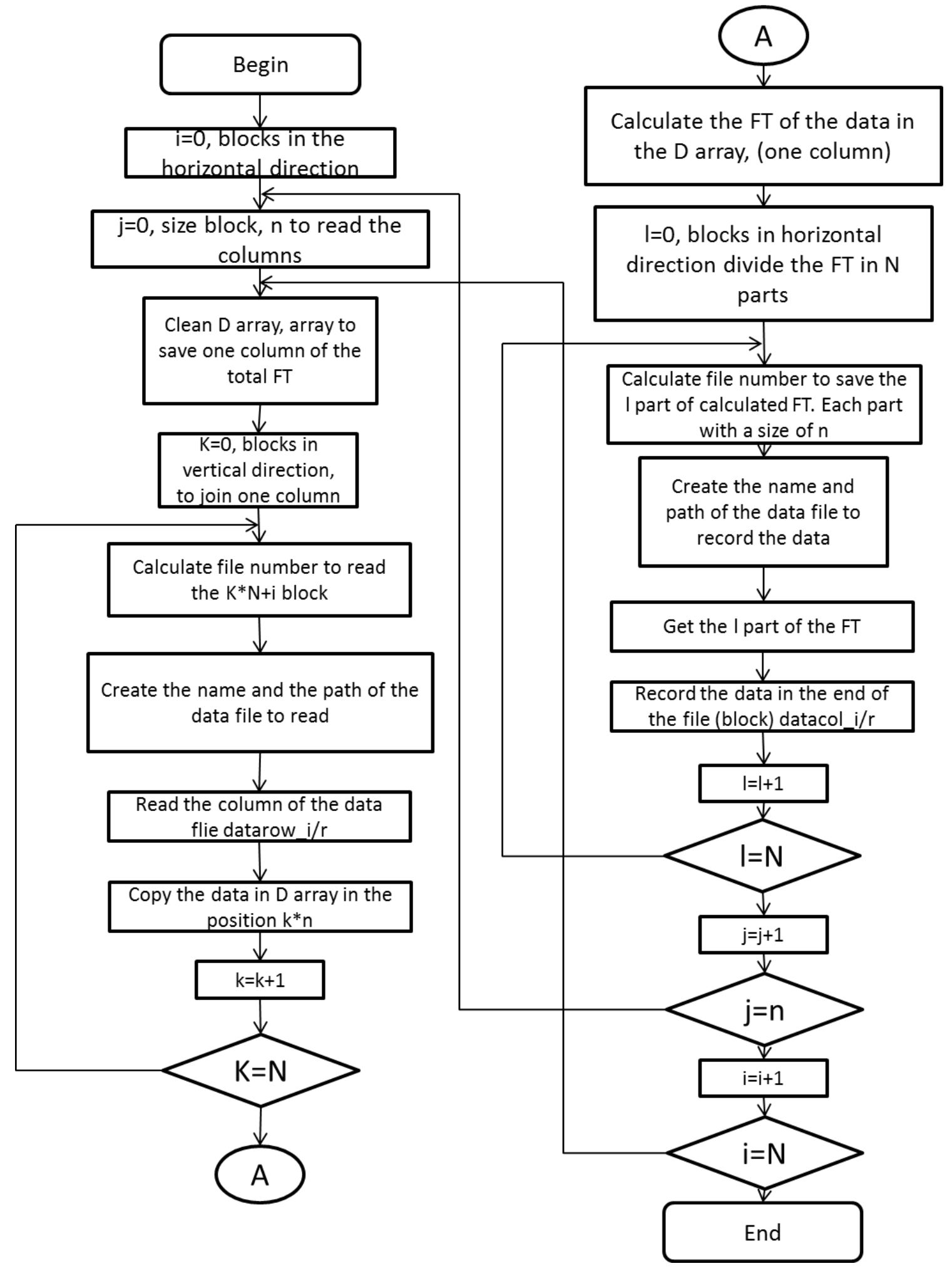




\section{F4}

The data is divided in $N \times N$ blocks of $n \times n$ size each block where $n \leq 512$. N should be multiple of 2 .

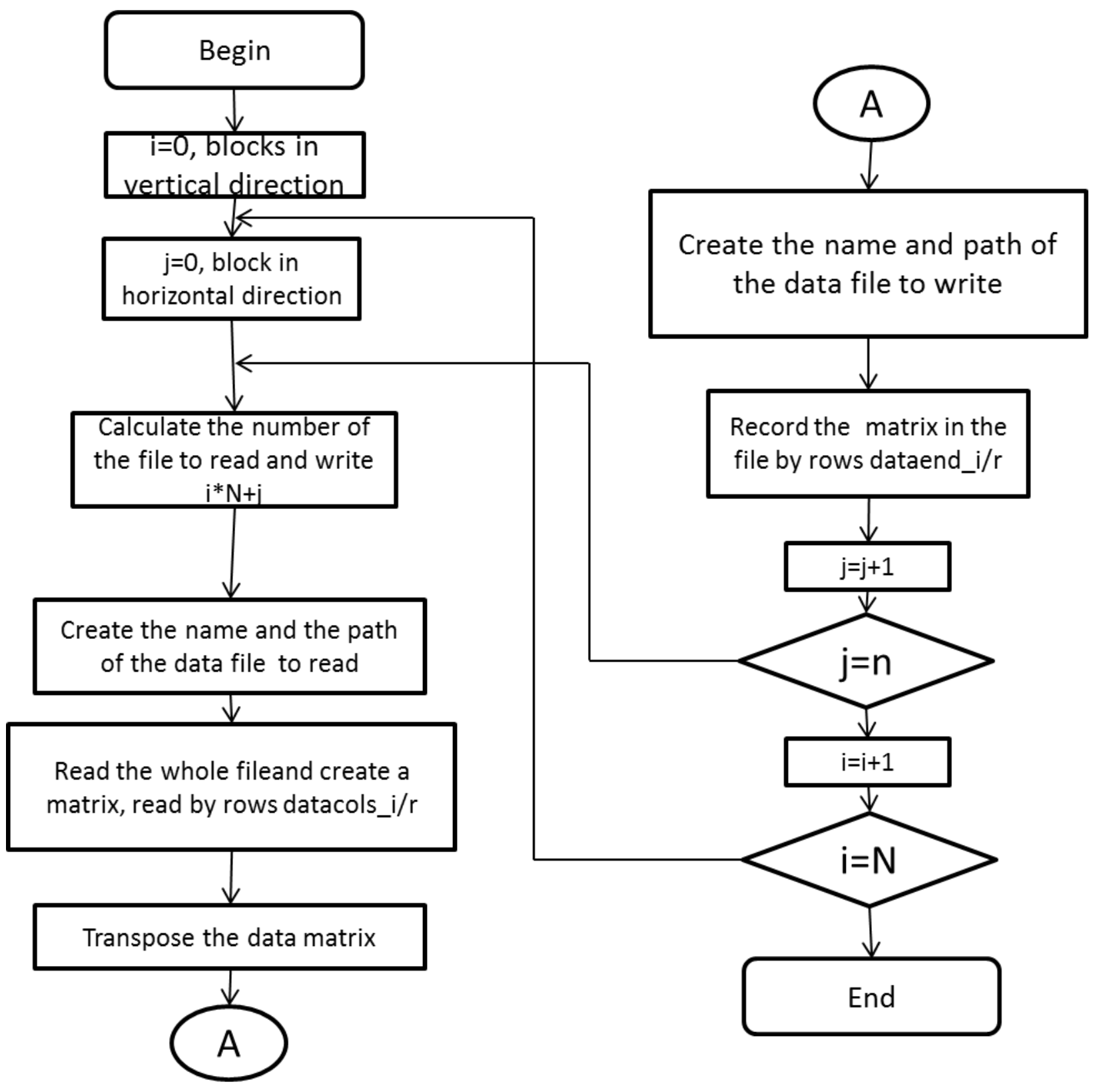




\section{F6}

The data is divided in $N \times N$ blocks of $n \times n$ size each block where $n \leq 512$. N should be multiple of 2 . The total Fourier Transform is divided in 4 parts, 2 horizontal, 2 vertical.

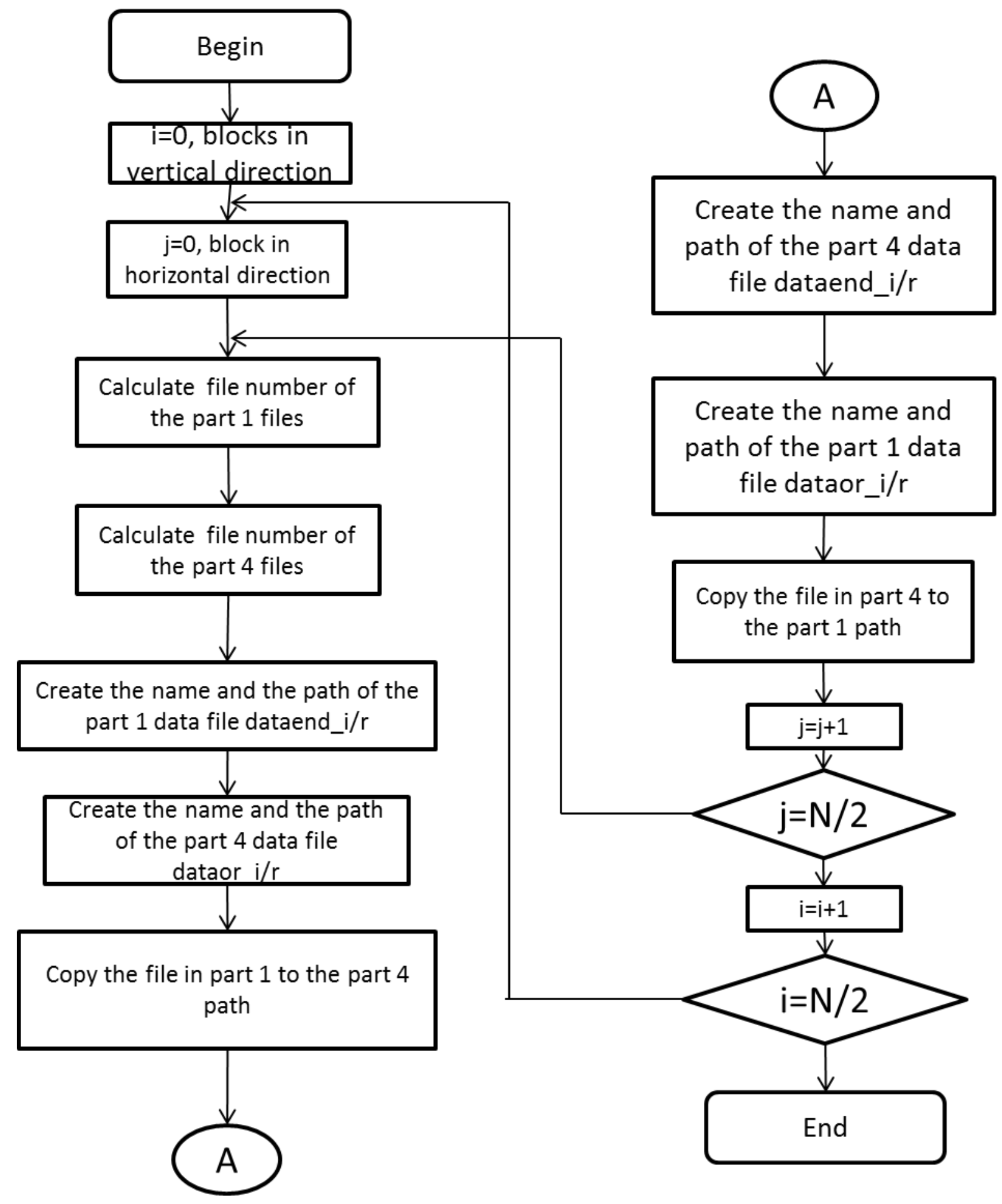


The data is divided in $N \times N$ blocks of $n \times n$ size each block where $n \leq 512$. N should be multiple of 2 . The total Fourier Transform is divided in 4 parts, 2 horizontal, 2 vertical.

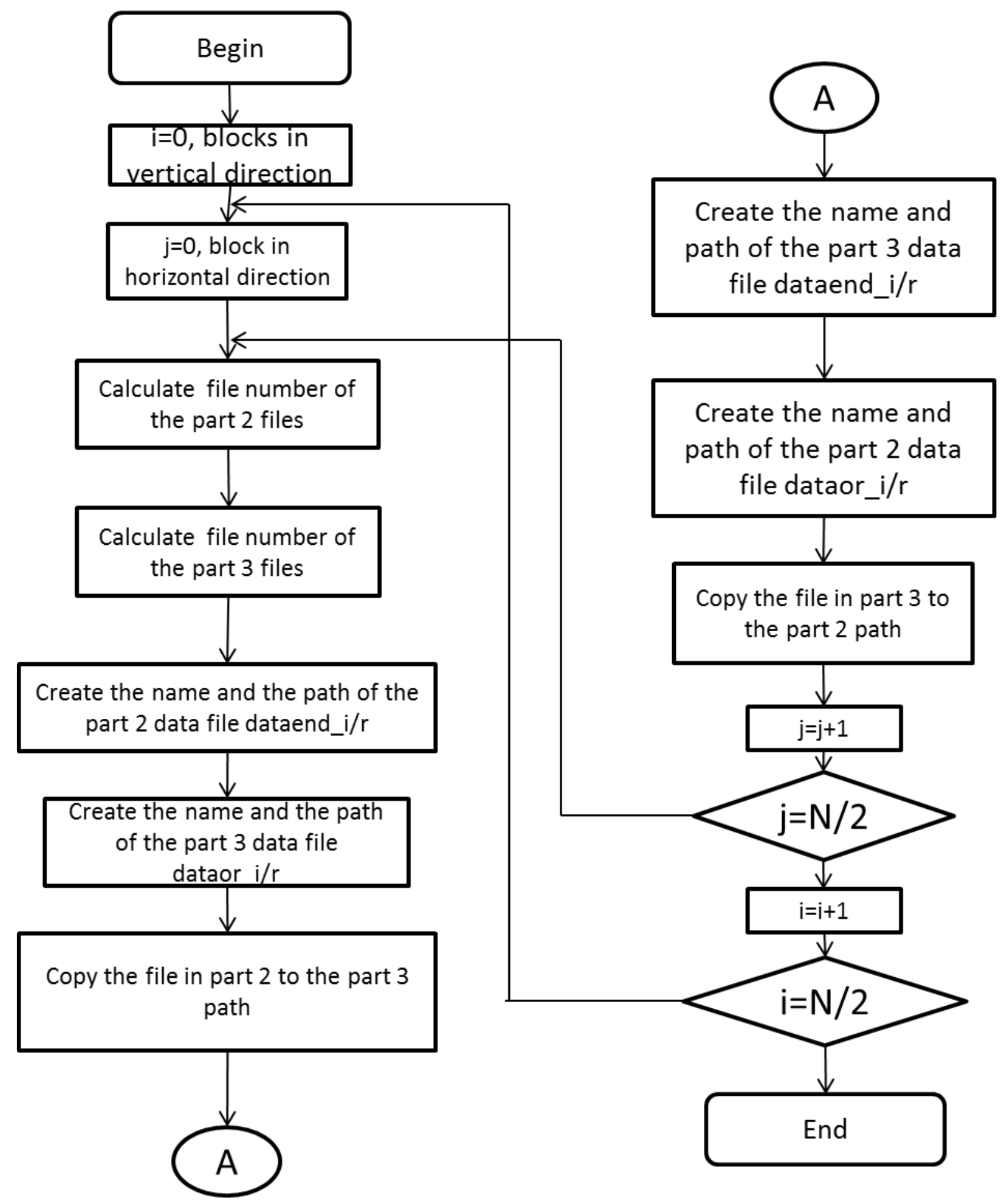


The data is divided in $N \times N$ blocks of $n \times n$ size each block where $n \leq 512$. N should be multiple of 2 .

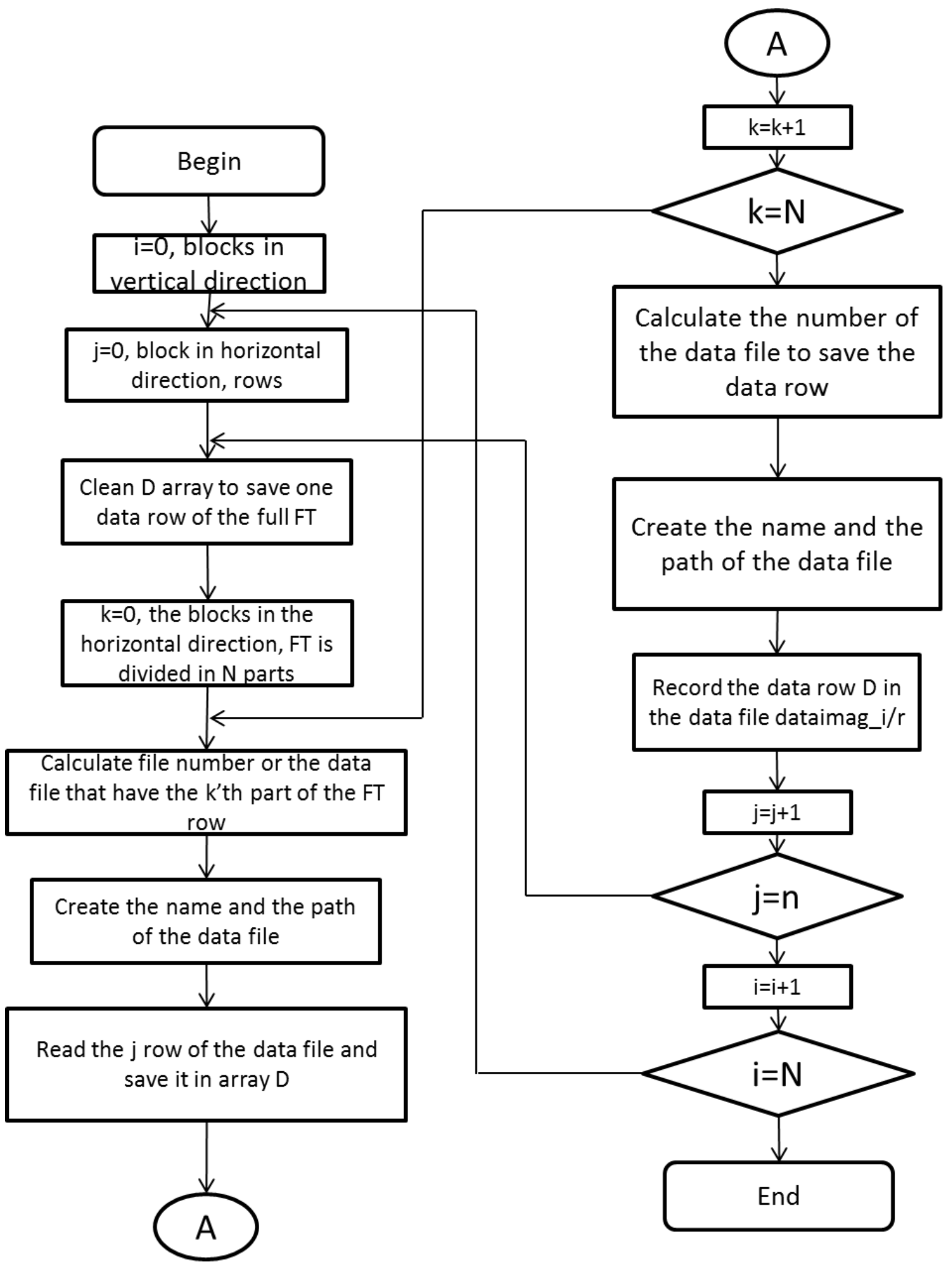




\section{F10}

The data is divided in $N \times N$ blocks of $n \times n$ size each block where $n \leq 512$. N should be multiple of 2 .

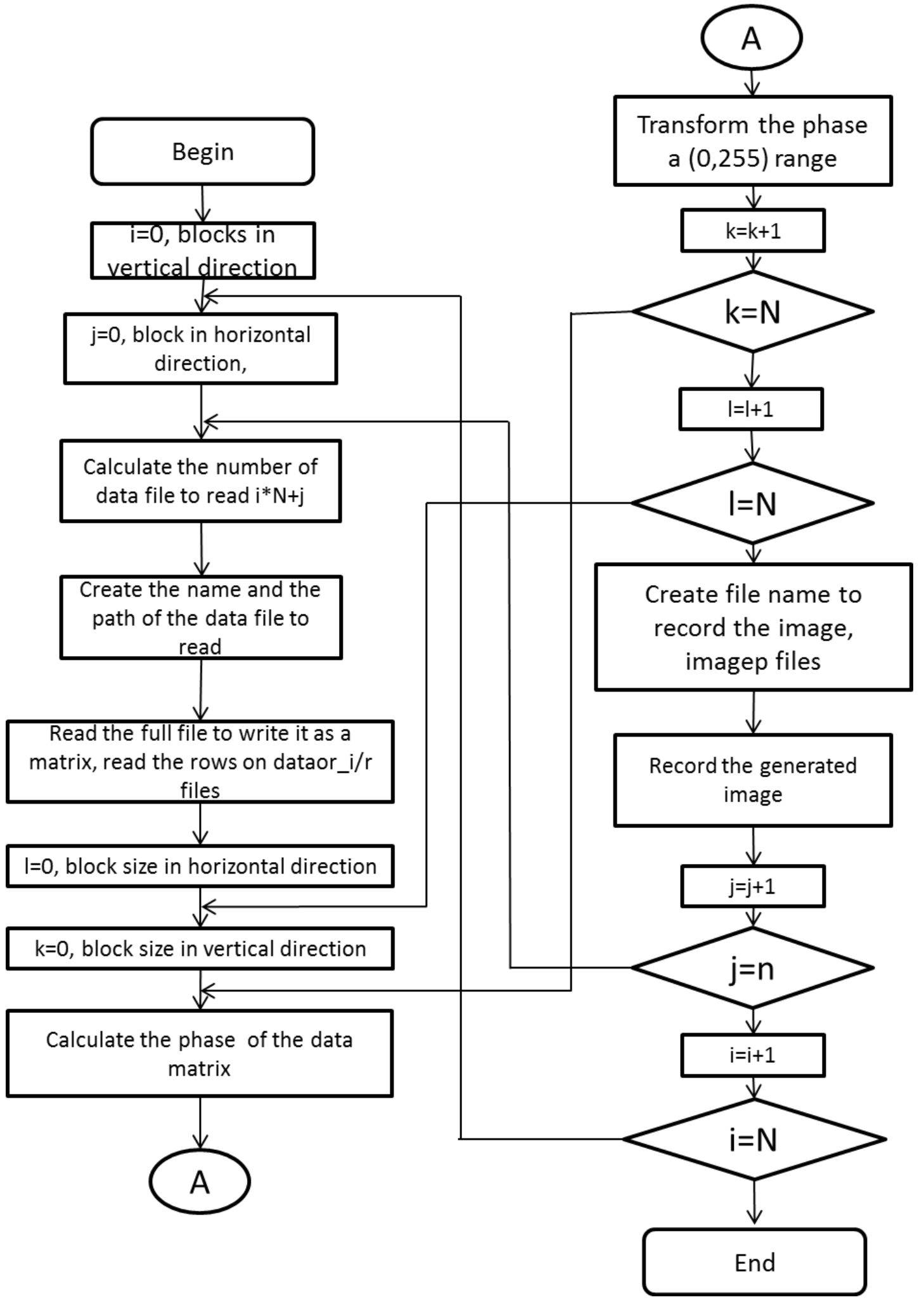




\section{F11}

The data is divided in $N \times N$ blocks of $n \times n$ size each block where $n \leq 512$. N should be multiple of 2 .

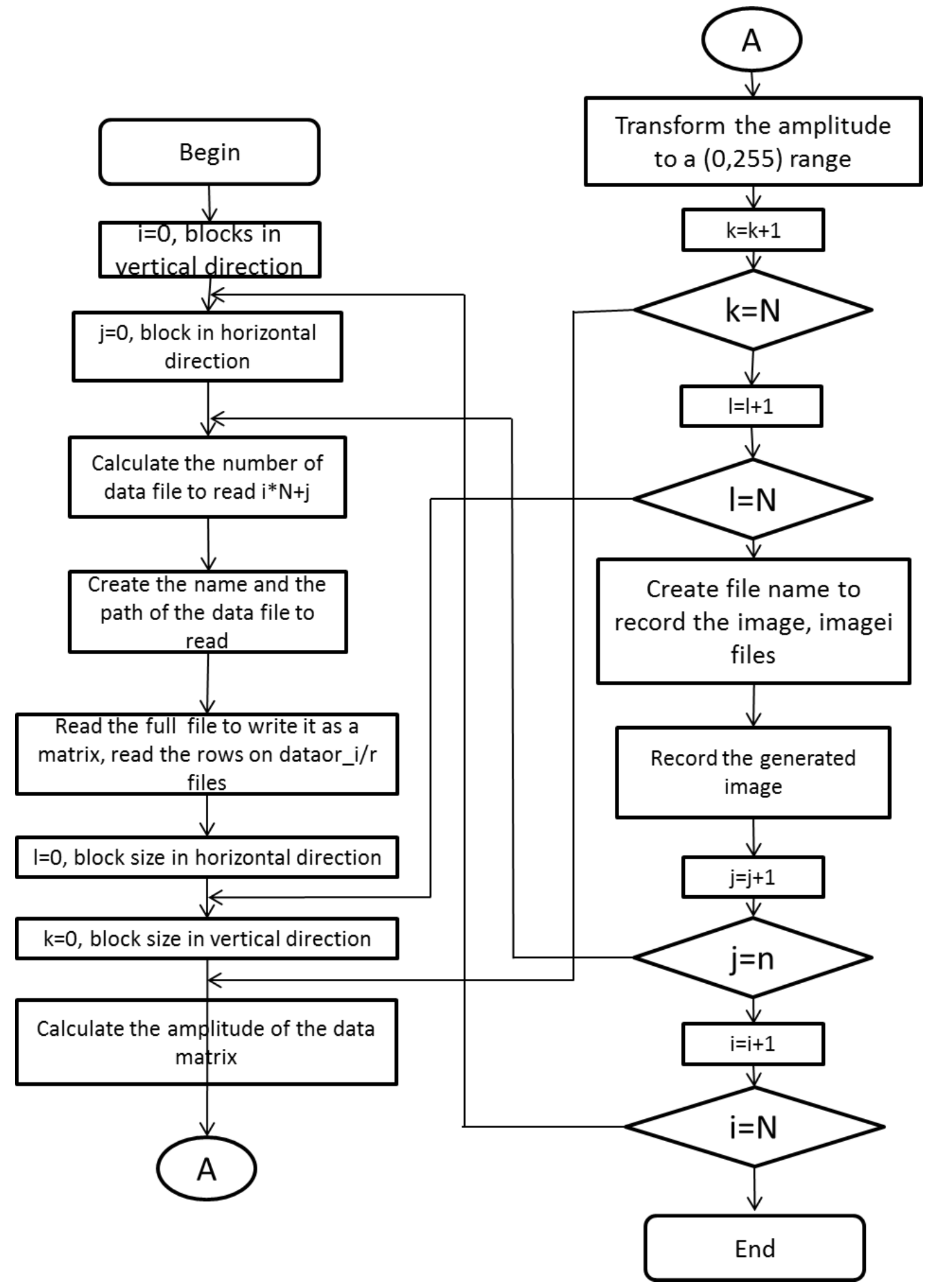




\subsubsection{Experimental Result}

The equation 3.2 includes the aperture function $P\left(x_{i}, y_{i}\right)$ limited in $y$ direction, a key component of rainbow holography and its existence allows the hologram to be seen in a white light illumination ambient (15). Also, $P\left(x_{i}, y_{i}\right)$ function provides encode perspective views by shifting its position in $x$ direction (Fig. 3.5a). Eight perspective images of $256 \times 256$ pixels were used in the experiment to obtain a $10 \mathrm{~mm} \times 10 \mathrm{~mm}$ color computer generated digital 3D Holographic Stereogram, as shown in Fig. 3.5b.
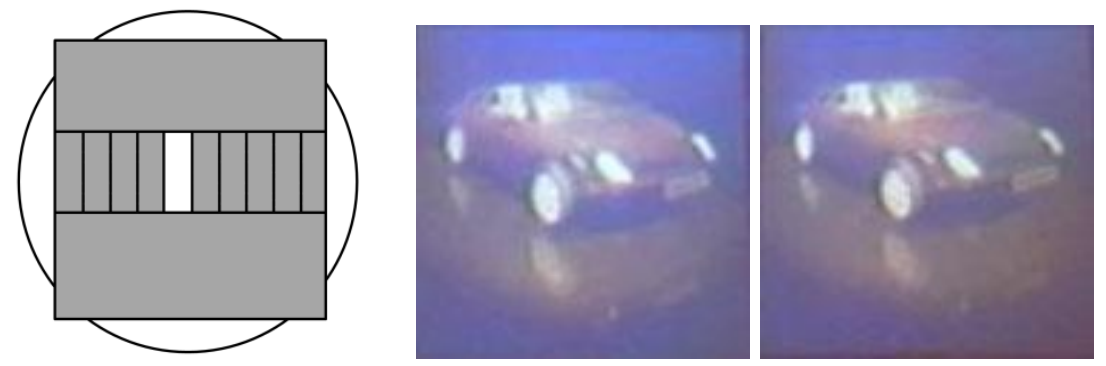

Fig. 3.5.(a)The aperture $P\left(x_{i}, y_{i}\right)$ provides perspectives views and white light CGH in reconstruction (left), (b) 3D color image in white light playback through adjacent reconstructed aperture/windows (right)

\subsubsection{Anamorphic one-step 3D true-color CGH}

Another simulated optical setup to obtain a 3D effect uses an anamorphic one-step-holographicstereogram (15), that consists of a lens setup with different focal length on the $\mathrm{x}$ axis $\left(f_{x}\right)$ and the $y$ axis $\left(f_{y}\right)$, respectively. As shown in Fig.6, the objective of this optical setup is to horizontally focus a 2D image on the focal plane $f_{x}$, and vertically focus on the focal plane $f_{y}$. In the real optical system, the perspective images are projected and focused to real images right behind the lens setup. In our digitized mathematic model, it is assumed that perspective images are propagated from the plane right after the virtual lens setup as shown in Fig. 3.6(above). The wavefront is transformed in $x$ and $y$ directions with $f_{x}$ and $f_{y}$ focal length, respectively. As a result, images are formed as a vertical line on $f_{x}$ focal plane, and a horizontal line on $f_{y}$ focal plane, respectively. 
The modeled setup can be expressed by Eq.

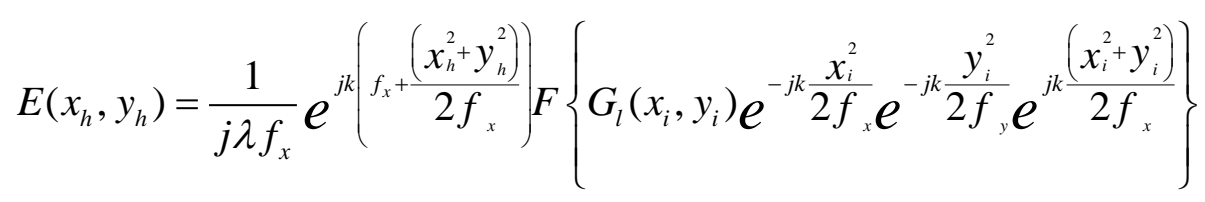

where $G_{l}$ is the bi-dimensional function containing one of the perspective views of the scene to encode, $\lambda$ the wavelength of the source that will be used on reconstruction. The line formed in the $f_{x}$ plane is then recorded on the hologram. Changing the design for a different view of the same object will produce multiple slit views of the object, which can be recorded lined one after another until a window of slits from the object is formed, these resulting in another case of autostereoscopic 3D hologram.

The above CGHs data has been calculated in such a way that the calculation and the printing can be simultaneously processed almost at the same time, so it can be called real time calculation. This was achieved with a PC running windows XP and a processor with a speed of $1.6 \mathrm{GHz}$. The complete writing process took two hours to complete and make a $10 \mathrm{~mm} \times 10 \mathrm{~mm}$ sample with the resolution described in the optical setup. The 3D stereoscopic effect was recognized through the insertion of a detecting screen right after each element hologram $(\mathrm{CGH})$ with a laser beam illumination as shown in Fig. 3.6(below). The reconstructed image is distorted into different shape until focused into a line shape where the location is to put pupil for observation.

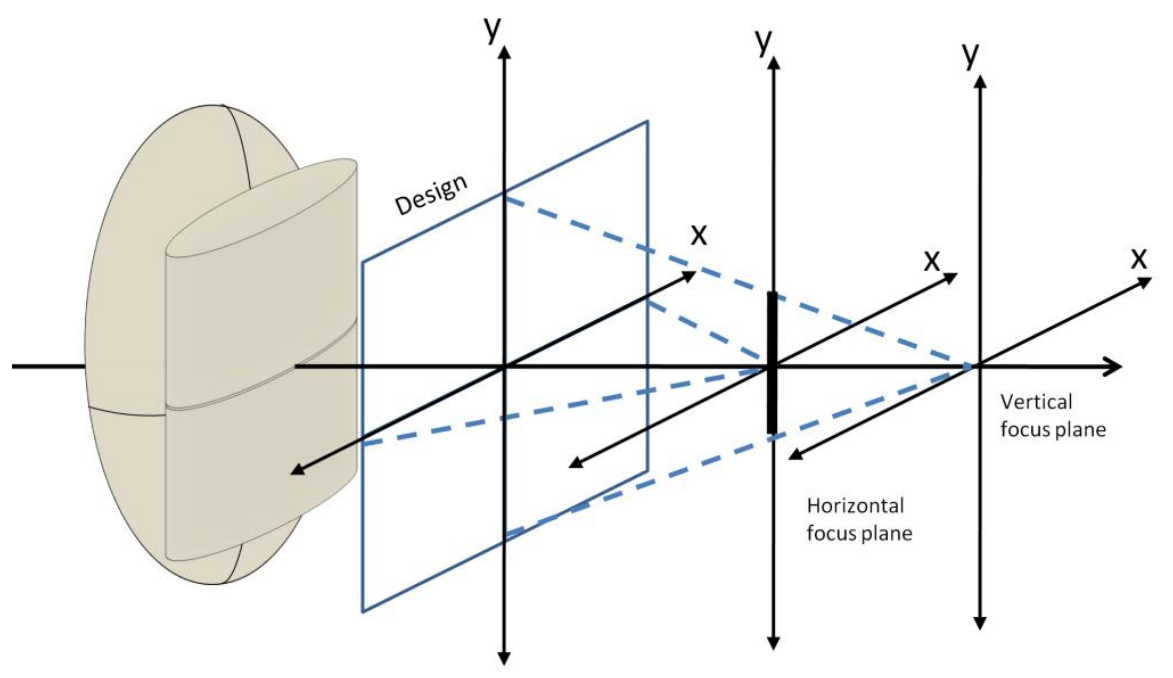




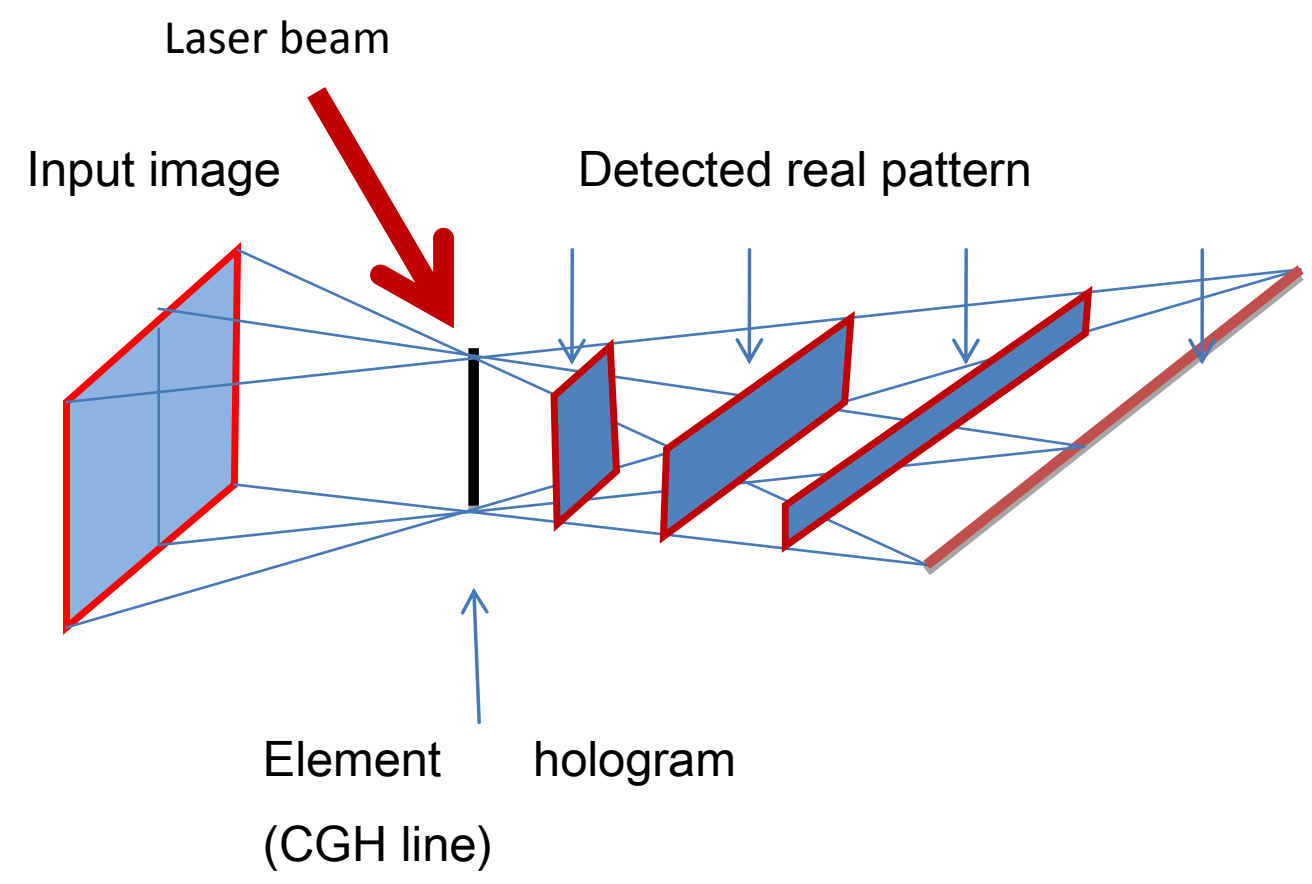

Fig. 3.6. Anamorphic lens model for recording a 1-step stereoscope CGH (above). The reconstructed patterns at different location after one element CGH (below)

\subsection{Kinoform}

A Kinoform is a phase only hologram in which only the phase modulation of an object wavefront is recorded to form a surface-relief profile. In this case, an assumption is made that the phases of the Fourier coefficients carry the majority of information about an object, and the amplitude information is entirely eliminated. The biggest advantage of a Kinoform is it's up to $100 \%$ diffractive efficiency through a perfect phase modulation. This is why it is very interesting to apply the system to create Kinoform CGH.

Considering the Fourier geometry again, the hologram is divided up into $N_{x} \times N_{y}$ cells, each representing one Fourier coefficient of the object. The amplitudes $\left|a_{p q}\right|$ of all Fourier coefficients are assigned value unity, and it is only the phases $\phi_{p q}$ that we attempt to encode in the hologram. The encoding is done by linearly mapping the phase range $(0,2 \pi)$ into a continuum of gray levels displayed by an output device such as a photographic plotter. The gray-level transparency obtained from this process is well enough controlled to assure that the complete phase range $(0,2 \pi)$ is exactly and proper- 
ly realized by the transparency, an excellent image can be obtained in the Fourier plane of the Kinoform.

Fig. 3.12(f) shows an example of a pre-calculated Kinoform pattern where the smallest element unit occupies about 0.4-0.8 $\mu \mathrm{m}$ hologram size. After developed, a laser beam illuminating from a normal direction of the hologram, an expected pattern with some noise could be recognized. The noise is considerable from given the lack of dynamic range control on the photo-resist. This causes errors in "phase matching" over the $(0,2 \pi)$ interval.

\subsection{Contour lines projection}

Apart of the CGH application mentioned above, another example of how to apply the system can be the encoding of contour lines projected from a given 3D model. The contour lines calculation makes a projection of a sphere, over a plane every time the sphere reaches a height multiple of $\lambda$, similar to a zone binary zone plate. 


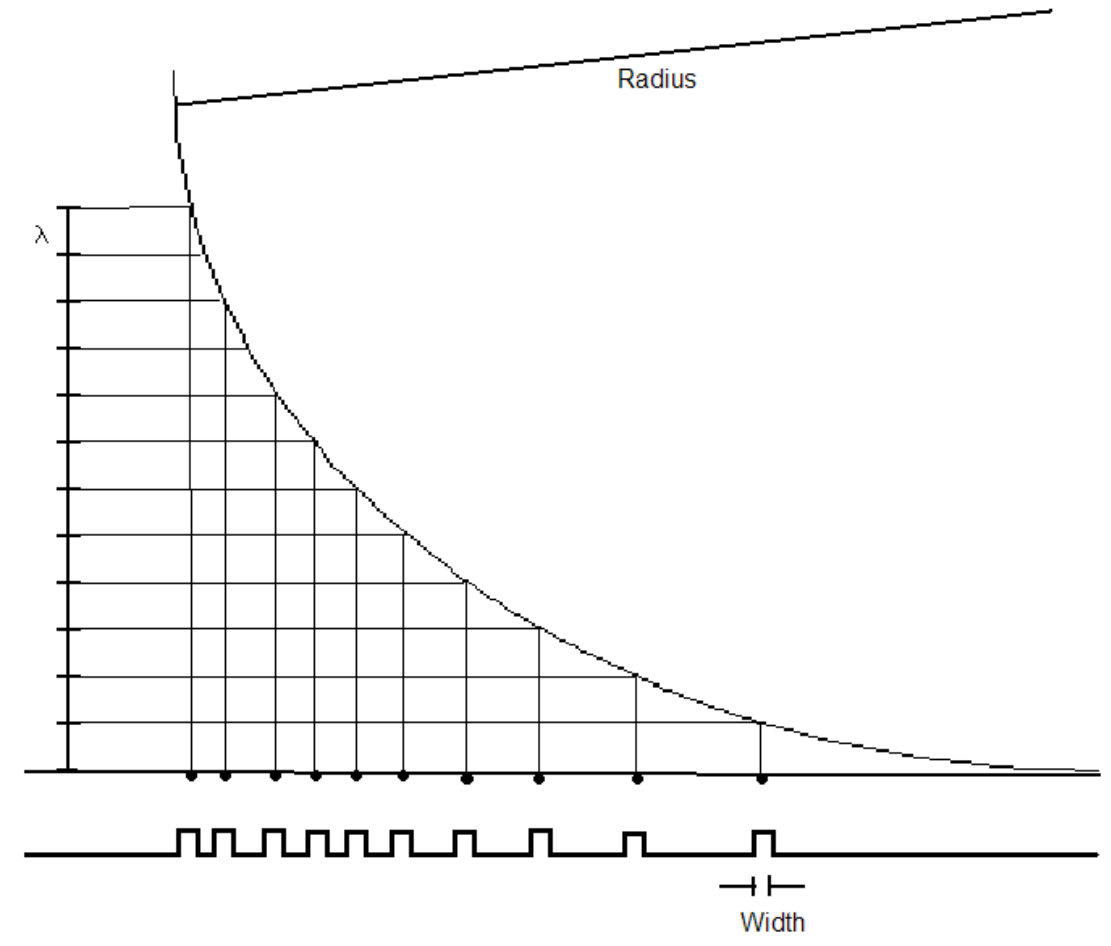

Fig. 3.7. Sphere Projection

The calculation program provides us to control the radius of the sphere and the width of the point of intersection. It also allows us to control the number of shots of 256 pixels that the image is divided by. In the program, it has two tabs, the first one, Corner Sample, allows us to see how the top left shot is going to look before using the second tab, Run Complete Set.

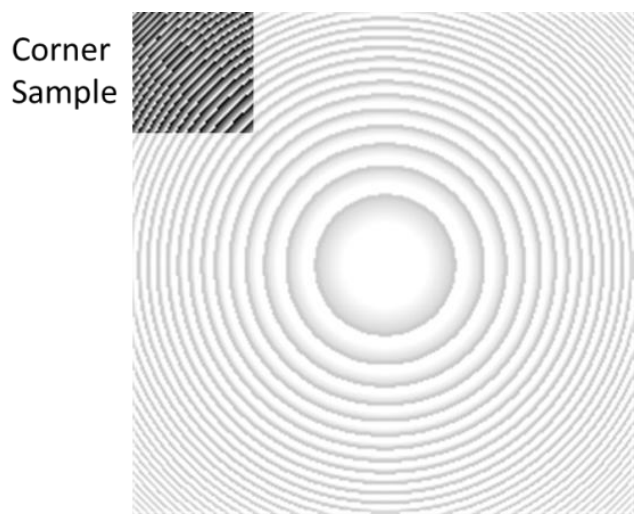

Fig. 3.8. Corner Sample 
When a complete set is calculated, a reference wavefront can be added to encode the sphere in an interfEerence pattern where the angle $\theta$ is provided as a parameter. The equation

code the hologram beam, which has maximum value each time $h(r)$ is a multiple of $\lambda$, and $h(r)$ is the height from the surface to the object (Fig. 3.9).

$$
O(r)=\cos \left[\frac{2 \pi}{\lambda} h(r)\right]
$$

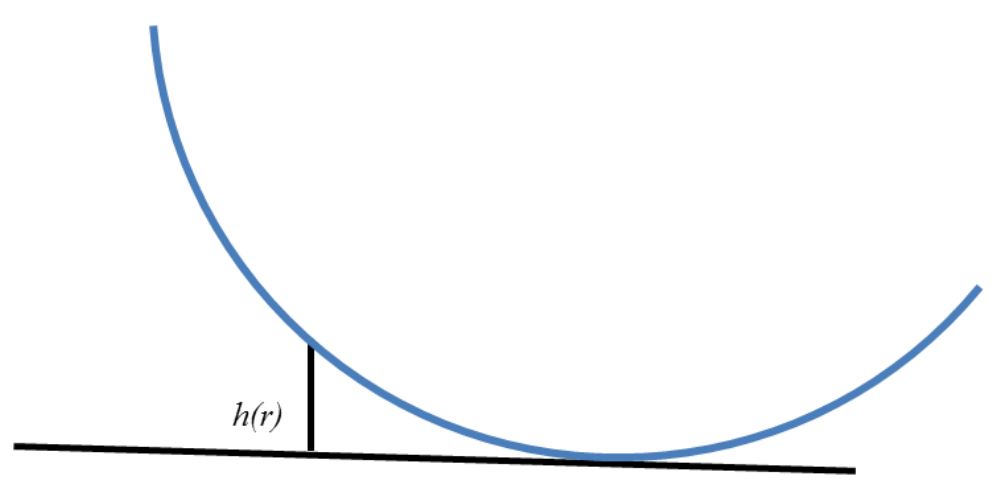

Hologram plane

Fig. 3.9. Distance $h(r)$ to the hologram plane

The reference beam is encoded similar with the equation of a plane wave:

$$
R(y)=\exp \left[\frac{2 \pi}{\lambda} y(\cos (90-\theta))\right]
$$




\subsection{Display/Security relief/rainbow Hologram}

In general, the methods for recording rainbow holograms can be classified as two-step methods and one-step methods, respectively. In the first method, a Fresnel hologram is prepared then in the plane of the real image reconstructed from the Fresnel hologram, the final plate is placed and exposed to record the wavefront from a horizontally limited slit. The viewer can view the image of the object thought the slit image. When white light is used as a source in the reconstruction process, each wavelength will reconstruct a wavelength dispersed slit in the vertical direction.

For the one-step method, couple of ideas have been proposed and used. For example, a lens is usually used to make a real image of the object in the plane of the holographic plate (16). Another one-step method has reported that can achieve a large format holographic stereogram using an anamorphic lens set (17).

In the conventional holograms, the objects are recorded in three dimensions. Each point of the holographic plate receives light of each point of the object. So we can consider that in each point of the plate is recorded a perspective view of the object. In the reconstruction process each point can reconstruct a perspective view of the object so we can see the object with a three dimensional feel (17) (18).

\subsubsection{Multi-optical features recording}

As described above, one of the important capabilities of the system is its application in security. Fig. 3.11 presents various data patterns that were calculated based on a single 2D grayscale look-up map. Fig. 3.12 presents the white light reconstructed image of the sample. The non-diffractive text/logo will measure $2 \mu \mathrm{m}$ in height when printed, with a 25000 dpi image resolution. This feature is detected only under a 500X microscope. The 1500 dpi full-color plain grating 32 views 3D color image with specklefree effect shows high quality and brightness.

To obtain this result, a dedicated processor makes the necessary calculation based on a 2D grayscale map and simulated optical data transform the result into fringe patterns and display it on SLM1. The same process is done to calculate an $M \times M$ cell array. Each cell can be further sub-divided into smaller parts to reach the optical limitation, as seen in Fig. 3.10. 
As shown in Fig. 3.11, the 2D grayscale image can be designed as a look-up map that indicates the optical feature corresponding to the output hologram. Therefore, it is easy to record various patterns in the same output work, not limited to fringe patterns but also can be combined with features like micro text, logotypes, etc. Examples of features include a 2D (2-dimensional) plain grating image, 3D (3Dimensional) grating image, a Kinoform pattern (16) and a CGH (Computer Generated Hologram). A small gap between adjacent exposures depends on the precision of the XY stage movement and is restricted to sub-micron order in this setup.

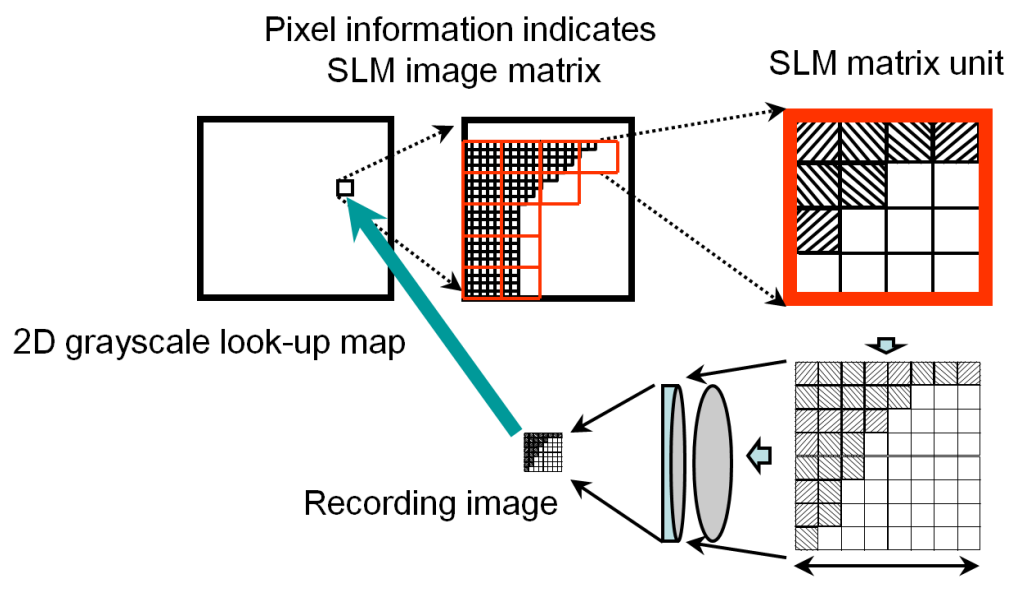

Full SLM active matrix

Fig. 3.10. Diagram of grayscale map transformation to recording image

(a)

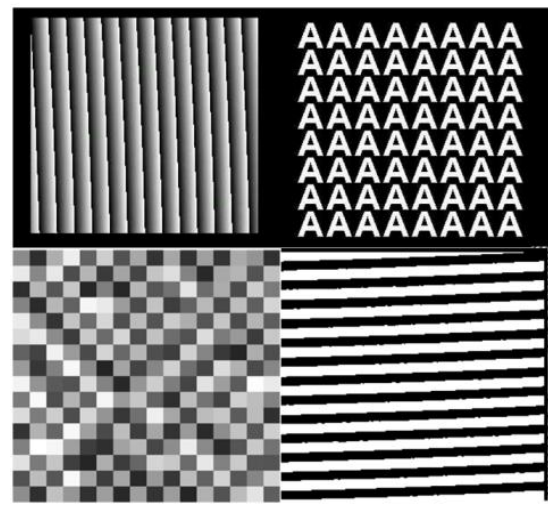

(f) (e) (c)

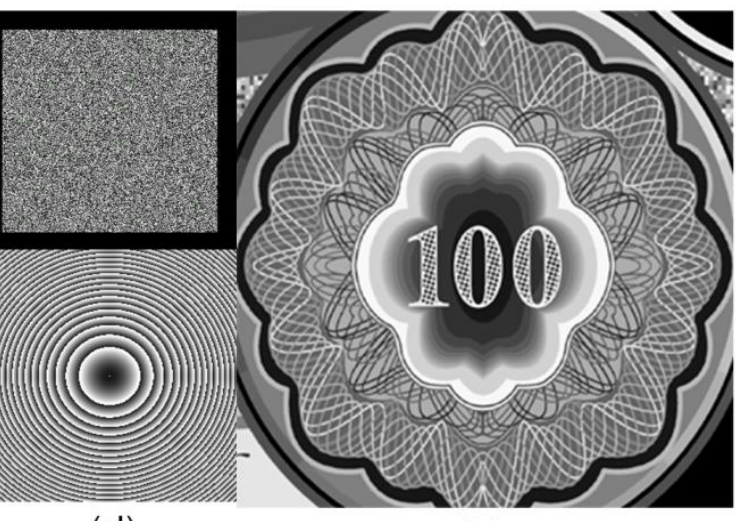

(d)

(g)

Fig. 3.11. Example patterns are calculated and directly projected on to photo-resist material based on single 2D map (g). From top-left clockwise direction; (a) blazed grating (b) non-diffractive micro-text (c) directional diffuser $(d)$ micro-lens (e) plain gratin 


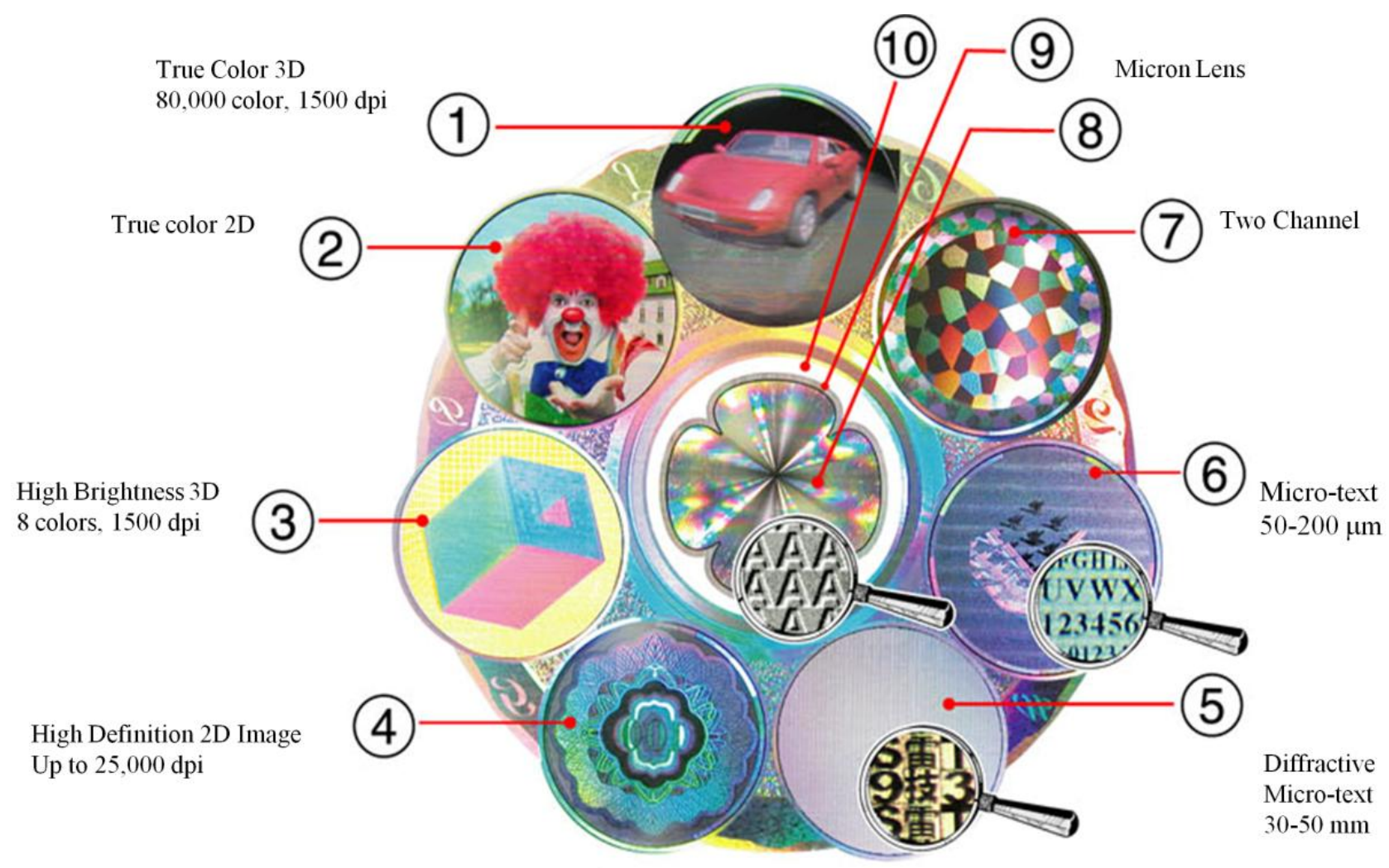

Fig. 3.12. Experimental sample that consists of various optical data directly printed through single well-designed 2D grayscale loop-up map

(Thanks to T-Security Inc. Taiwan for providing the art design and photography shown here) 


\section{Lippmann holographic recording process}

\subsection{Recording setup}

The other mode of the system is the one who will write true-color Lippmann hologram. Similar to the relief type (rainbow hologram) recording mode, calculated images (if necessary) or perspective images are displayed on SLM1 and further reduced in size with approximately 50X magnification, then imaged over the recording material plate.

It is simple to switch the optical setup from relief hologram recording mode into reflection type hologram recording mode as shown in Fig. 4.1. In this mode, the zoom lens unit can be automatically adjusted by computer control to shift the focal plane of the SLM1 image to the plane behind of the plate. The focal plane shifting obtains a fine homogeneous energy distribution on the volume emulsion for a better dynamic range control to the material sensitivity. The propagation of the wavefront coming from the SLM and a reference beam form a volume fringe pattern as a holographic element. The size of the element is limited by the energy distribution of the object and the reference beam. The maximum viewing angle of the reflective hologram is determined by the $N A$ of the optical setup, that is 0.45 in this case and achieve about 60 degree viewing angle.

A light source chamber containing three color $(633 \mathrm{~nm}, 530 \mathrm{~nm}, 457 \mathrm{~nm})$ lasers is introduced in the same optical path to obtain a full-color hologram. In this recording mode, instead of the random phase modulation in digital rainbow recording mode (Fig. 4.1), it is feasible to apply phase information to enhance the image quality by modulating the phase on SLM2. 


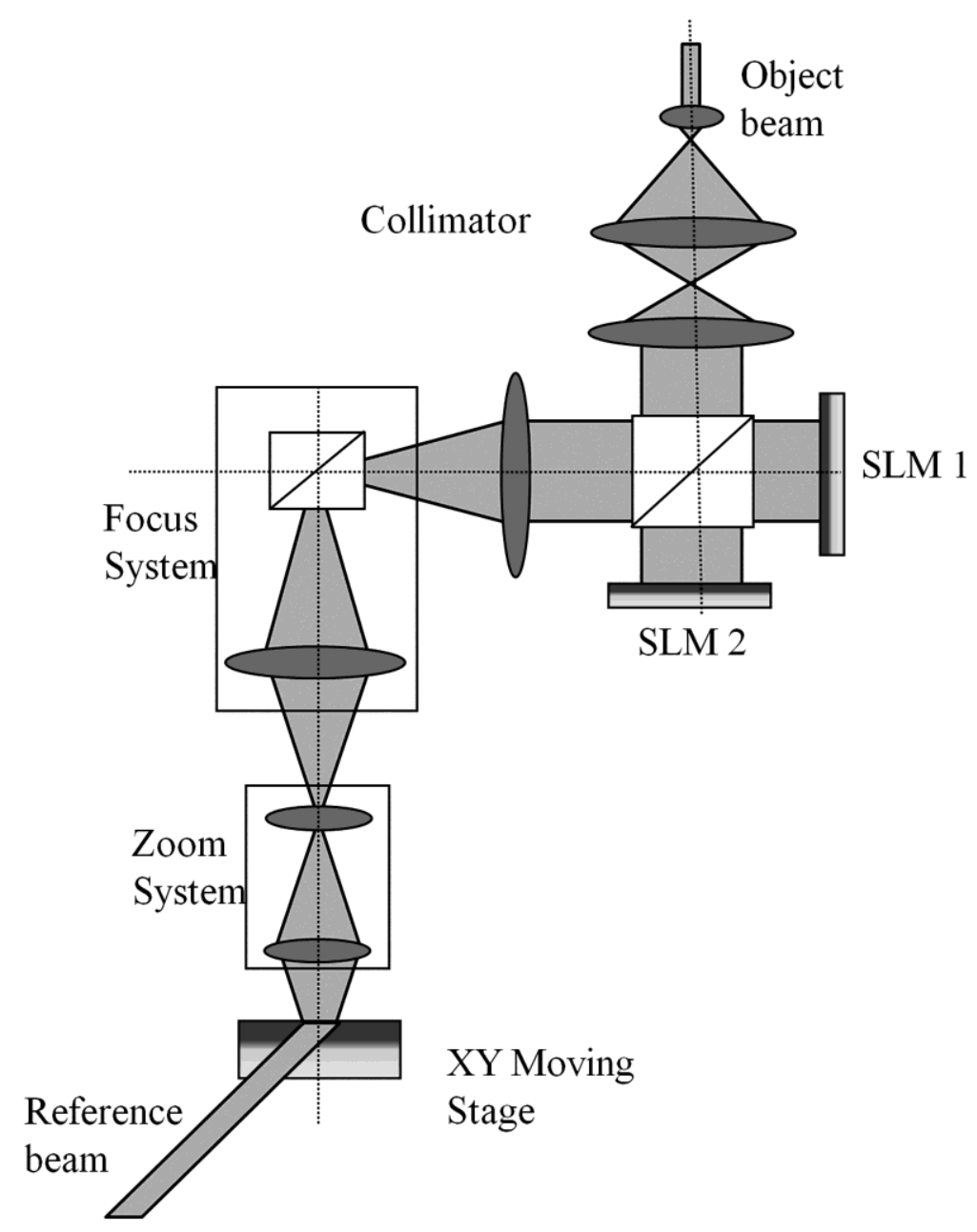

Fig. 4.1. Lippmann type hologram recording setup

\subsection{Image processing}

An image processing to shift the reconstructed image plane for horizontal only holographic stereogram was proposed in 1991 by Dr. DerKuan Kang (15) and further extended into parallax process as in Fig. 4.2. In this research, the image processing is further extended into parallax views through a new geometric model consideration. 


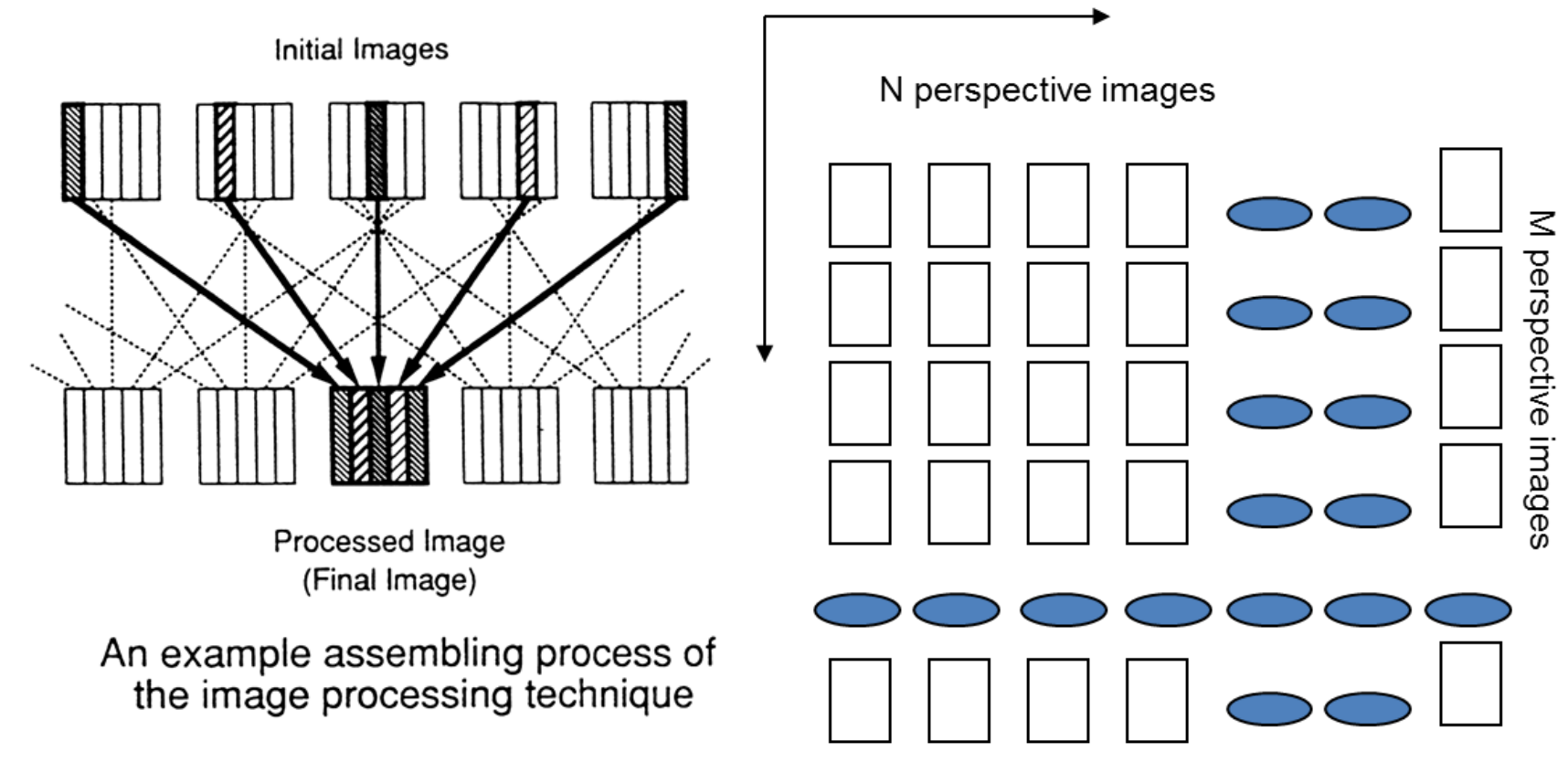

Fig. 4.2 Horizontal only image processing holographic stereogram (left), parallax view image processing (right)

The record of the holographic stereogram consists on displaying a series of parallax views taken from a model on a holographic plate, each view called a holographic primitive. Each pixel inside the displayed parallel view represents a ray of light coming from the modeled object. The complete holographic primitive consists on a whole bunch of these rays that arrive to a certain position $P(x, y)$ at a distance $z_{1}$ from the object, which corresponds to a photography of the object from that point. Each one of the rays will come from a different angle, and the maximum angle will be the aperture angle $\theta_{n}$. As seen on Fig. 4.3. 


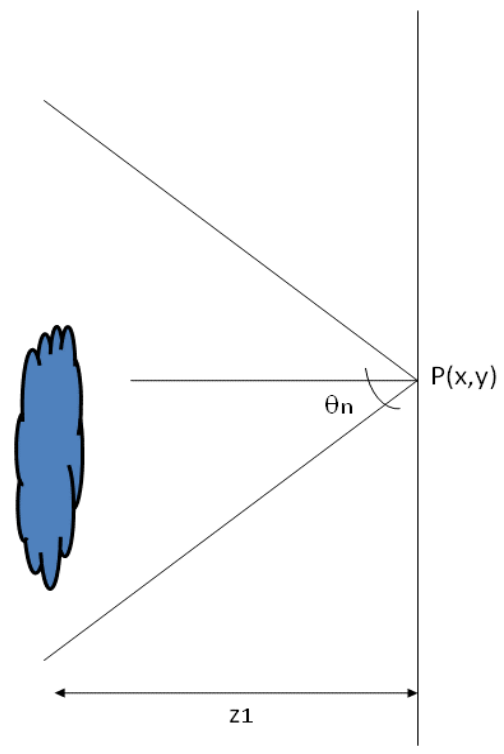

Fig. 4.3. Total hologram viewing angle

The record process continues by then recording the next holographic primitive on the hologram on its corresponding position. This continues until all the primitives are recorded. The number of holographic primitives needed depends on the final resolution we want the hologram to have. In a geometric standpoint the model distance to the camera shooting on the recording and the depth of the object inside the hologram in the reconstruction will have the same ratio as the total camera movement when recording the model and the total size of the array of the holographic primitives.

\subsubsection{Shift of the image plane}

Let's say on the reconstruction we want the object to be closer to the hologram plane than this ratio. We even want to put the object in front of the hologram plane instead of in the back. Because we are considering each primitive as a set of rays, we can rearrange them to make our goal possible.

All our holographic primitives are constituted by a set of rays, each of this represented by a pixel inside an image in our computer. This means that each one has different values for position in a plane and for RGB color. The position will define the ray direction following the equation (

), that can be ap4.1

plied in $x$ or $y$ axis of the hologram plane. $N$ means the number of pixels in a line inside the hologram primitive. $\theta_{n}$ is the aperture angle of the optical system that projects the SLM information into the holographic plate and $\mathrm{I}$ is the pixel number in that direction varying from 0 to $N-1$. 


$$
\theta_{i}=\tan ^{-1}\left[\left(1-\frac{2 i}{n}\right) \tan \theta_{n}\right]
$$

Even if we rearrange the order of all our data volume, the direction each ray cannot be changed, so the ray must maintain its coordinates intact inside a primitive. (Fig. 4.4)

Let's say the hologram plane it's at a distance $z_{1}$ from the recorded objects and we want to put the hologram plane at a new distance $z_{2}$. The ray that hits the hologram plane in point $P\left(y_{1}{ }^{\prime}\right)$ with an angle $\theta_{1}$, will hit the point $P\left(y_{1}{ }^{\prime}\right)$ at the new hologram plane distance. So if we have the information of that ray in a holographic primitive, we can build a new holographic primitive looking in our data volume for all the rays that will hit the point $P\left(y_{1}{ }^{\prime}\right)$. This can be done in an ordered way for all the new primitives we need, making a new set of them that will contain the same rays, but rearranged. In Fig. 4.4, we show an example of how two rays from our original data volume are reused in different hologram primitives, without modifying their angle and values properties.
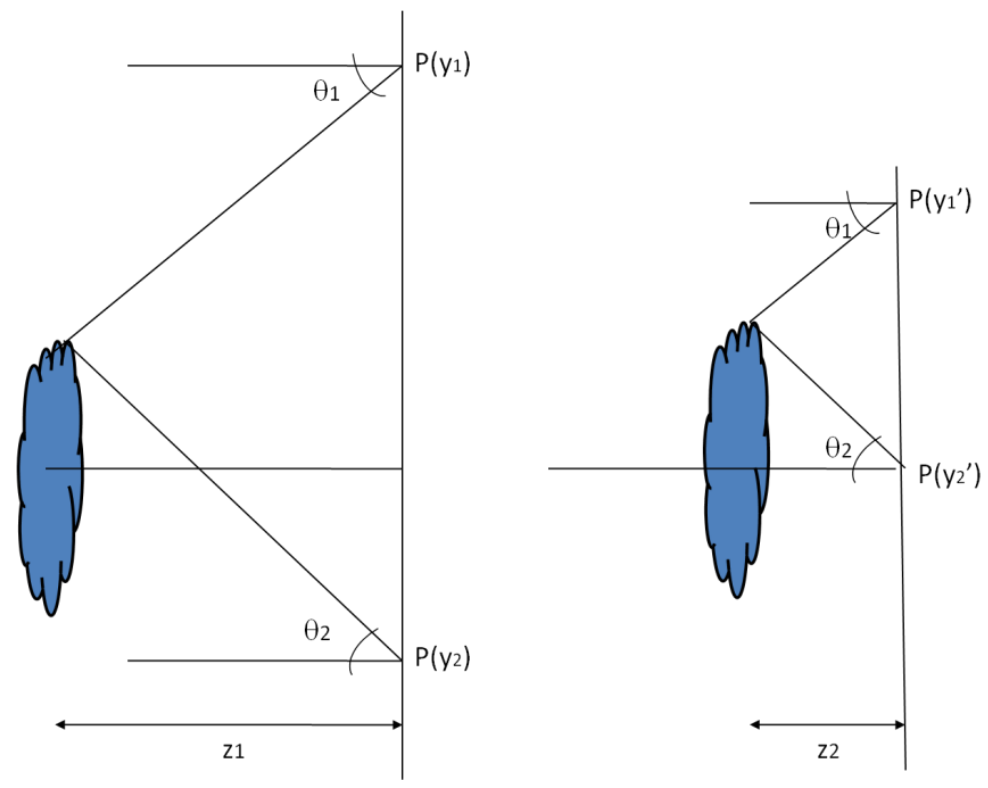

Fig. 4.4. A certain point inside the object is viewed only in a certain part of the hologram 

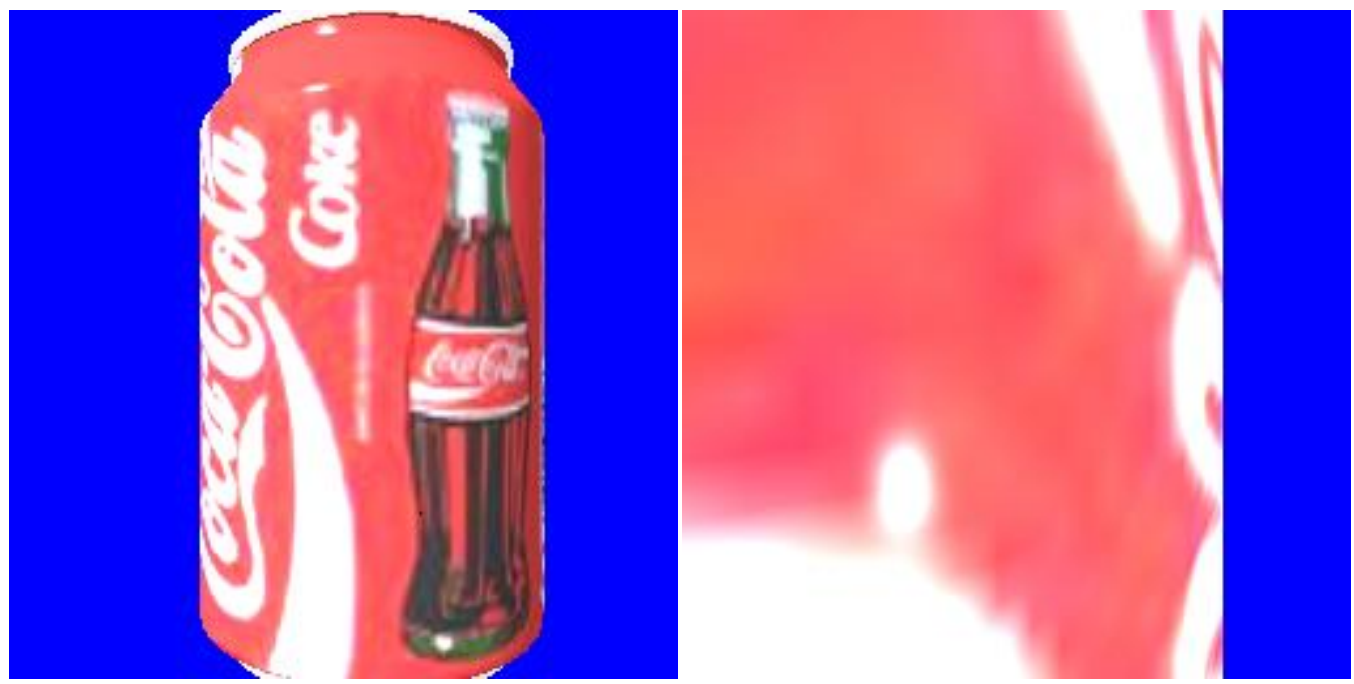

Fig. 4.5 An example of original perspective image (coke can, left), after processed using the new algorithm (right) which calculation is much faster and more flexible than traditional method

\subsubsection{Modifying the shape of hologram surface}

If the final shape of the printed hologram won't be a plane, but another kind of surface, when recording the object, we have something similar to Fig. 4.6, where from each point we have all the rays coming from a point with a recollection angle $\theta_{n}$ perpendicular to the surface.

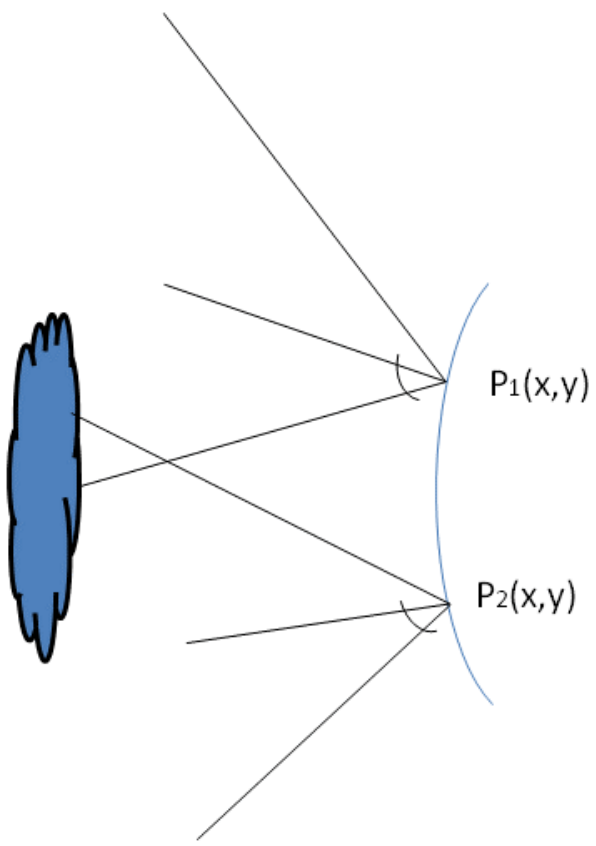

Fig. 4.6. A curved surface changes the object segment seen from a certain point 
If the acquisition method was our standard method of perspectives taken from moving a camera on a plane, as in the last section, we can take the pixels as rays and rearrange them to be on the position we need to simulate the geometry of our future surface.

This will simulate the curvature and differences in depth in the hologram. If the real hologram will be reshaped, then to contrast this, the reciprocate curvature and the negative depth will be recorded, so when the hologram is bended, the shapes reconstructed by the hologram will have the orientation needed.

Because the limitations of the system, we cannot record at an angle bigger than our capture angle, so we don't have the information of the arc that does not superimpose with the original arc. In Fig. 4.7, the original recording is contained by $\operatorname{arc} A P_{1} B$, but we need to record the rays coming from the $\operatorname{arc} A^{\prime} P_{1} B^{\prime}$, so the arc between $A^{\prime} A$ will be missing, and our viewing angle of the hologram will be cut.

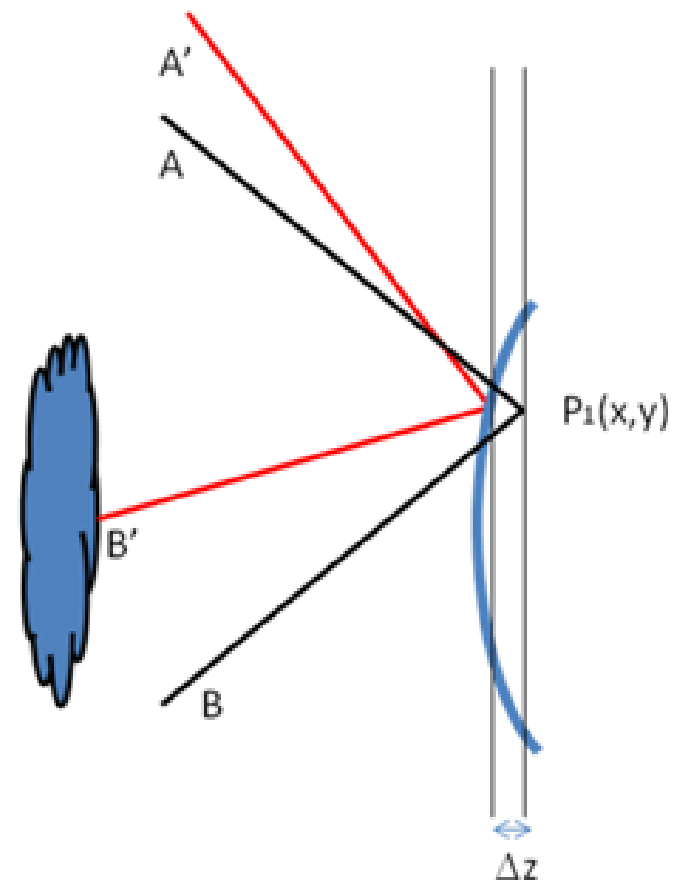

Fig. 4.7. Different view field over a curved surface 


\subsubsection{Removing lens distortion using a digital approach}

Distortion is one of the aberrations presented in systems with lenses and probably the biggest contribution to aberration. Because it is a radial distortion it can be expressed as a function that depends of the distance from the center of the image. The function goes from the distance in a non-distorted image $r$ to a distance in a distorted image $r^{\prime}$.

$$
r^{\prime}=f(r)
$$

We can express this function with its Taylor series:

$$
r^{\prime}=r+k_{1} r^{3}+k_{2} r^{5}+k_{3} r^{7}+\ldots
$$

Measurements have proved that a good approximation can be given by the first term only of eq.

4.3 This means we can express the function as a third grade polynomial:

$$
r^{\prime}=r+k_{1} r^{3}
$$

This formula allows us to distort an image. To compensate an image given a pre-distorted one, we need to calculate the inverse function and leave $r$ as a function of $r$ '.

$$
r=f^{-1}\left(r^{\prime}\right)
$$

Calculating from our previous approximation, using the Cardano method to solve third grade equations, we can determine a value for $r$ :

$$
r=\sqrt[3]{\frac{r^{\prime}}{k_{1}}+\frac{-\frac{r^{\prime}}{k_{1}}+\sqrt{\left(\frac{r^{\prime}}{k_{1}}\right)^{2}+\frac{4}{27 k_{1}^{3}}}}{2}}-\sqrt[3]{\frac{-\frac{r^{\prime}}{k_{1}}+\sqrt{\left(\frac{r^{\prime}}{k_{1}}\right)^{2}+\frac{4}{27 k_{1}^{3}}}}{2}}
$$

Now for each point in the new image we can calculate where it comes from inside the original image only by changing its pixel position to polar coordinates, taking the center of the image as the origin. To avoid having scale problem issues, we should first normalize the coordinates using the unit as the radius of the circle that circumscribes the image. After calculating the position in the original plane, if it 
is not exactly one pixel position, we can calculate the corresponding value using either bilinear interpolation or nearest neighbor algorithm.

We yet have the problem of finding the values from the pixels that comes from outside the original image area. This can be done calculating bigger images before, so the images farther than certain position are going to be discarded and only used to calculate the pre-distorted image. Another solution, but maybe not the best one is to display the center of the image only, were the values are known and cut the rest of it.

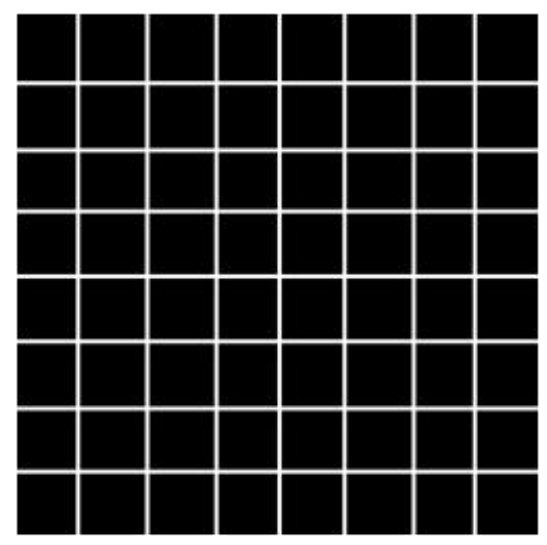

(A)

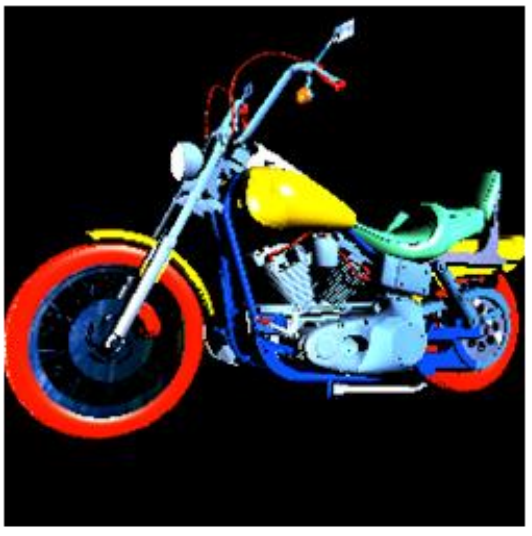

(C)

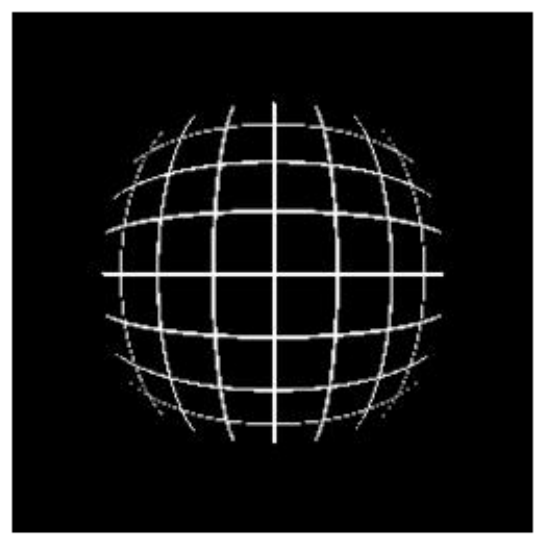

(B)

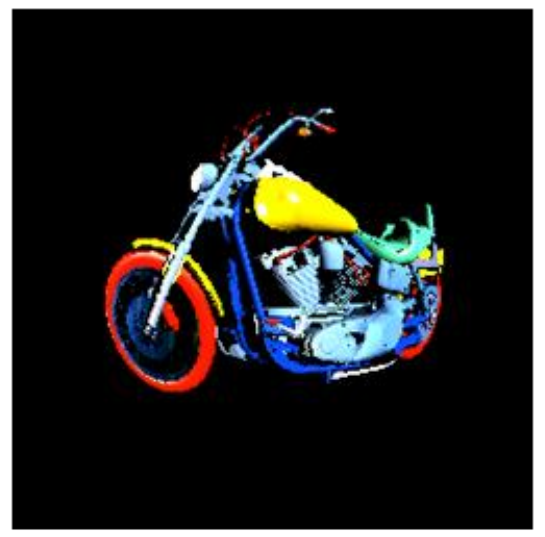

(D)

Fig. 4.8 Input images $(A)$ and $(C)$, Pre-distorted images $(B)$ and $(D)$ for compensating the lens aberration 


\subsubsection{Processed image sample}

The image processing algorithm is applied to a set of $256 \times 256$ holographic elements making a full parallax hologram. Each holographic element consisted of a $256 \times 256$ pixel image. The left image on Fig. 4.9 is an example of a centered perspective view. The processed image in the right is shown as a pre-distorted result after the image processing used for shifting the reconstructed 3D image around the hologram plane.

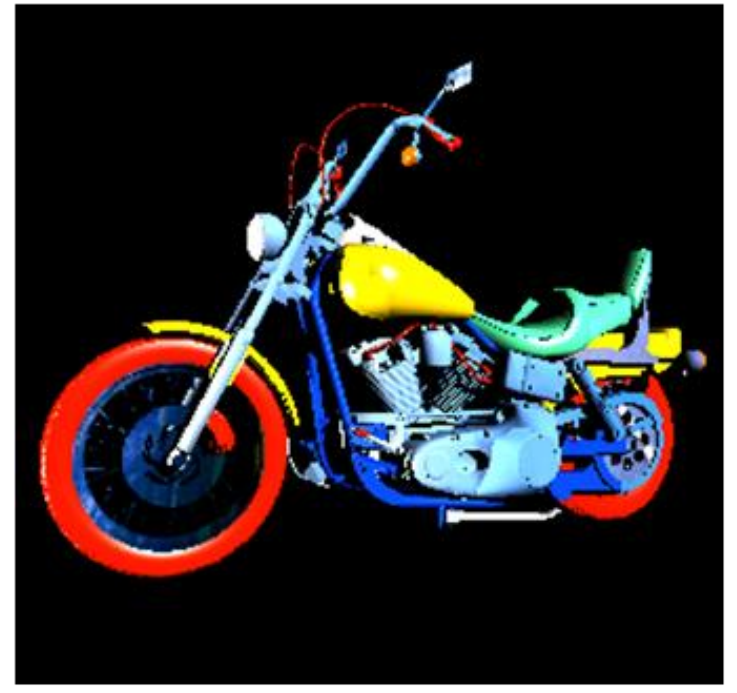

(A)

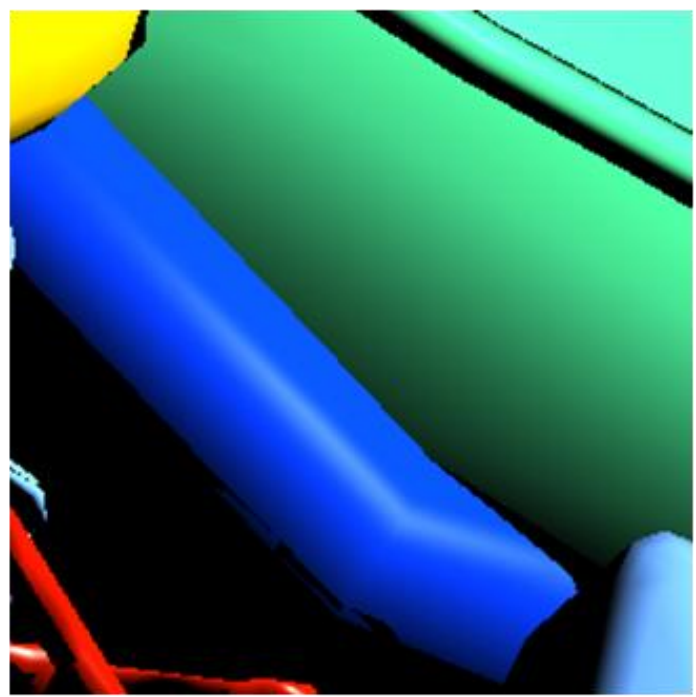

(B)

Fig. 4.9 Pre-distortion of a perspective view

\subsection{Thick material characterization}

There is a kind of volume type recording panchromatic material with extra-fine particle silver halide emulsion called PFG-3C, which provides a resolution of up to 5000 line/mm. This was the material chosen in the experiments.

The Efficiency-Energy relationship and the wavelength selection among color range are shown in the Graph 4.1 and Graph 4.2, respectively. 


\section{DIFFRACTION EFFICIENCY}

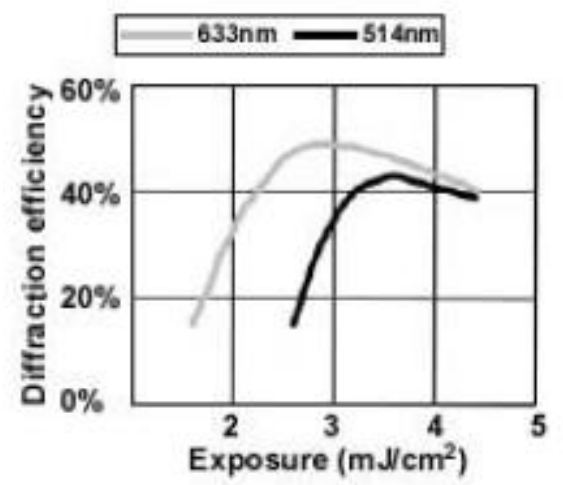

Graph 4.1 Diffraction efficiency graph

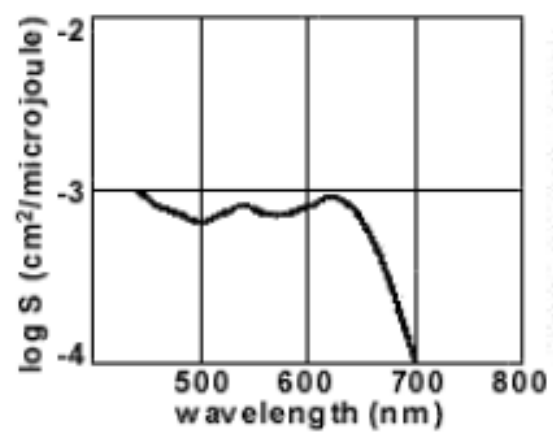

Graph 4.2 Sensitivity-exposure energy graph

\subsubsection{Spectral sensitivity}

Developing of these plates is done in a single process as shown below using developer JD-4.

Developing time: 10 seconds

Rinsing: 10 seconds

Bleaching: 80 seconds

Rinsing: 30 seconds

PhotoFlo: removing the plate carefully avoiding water drop and spills.

When making a volume hologram, it is imperative to pay attention to the following points: 
1. Vibration: This is normally introduced from the XY stage or/and lost fastening of optical components.

2. The simultaneous optical path for the three color exposure.

3. Polarization of each color varies its split ratio in different components.

4. Intensity ratio: Theoretically, the best reference-object beam intensity ratio should be $1: 1$, but because the difference in the beam profiles, a different ratio was found to be experimentally around $2: 1$.

Manual adjustment was used to ensure the correct alignment between the reference beam and the object beam using precision screws.

\subsection{Recording conditions}

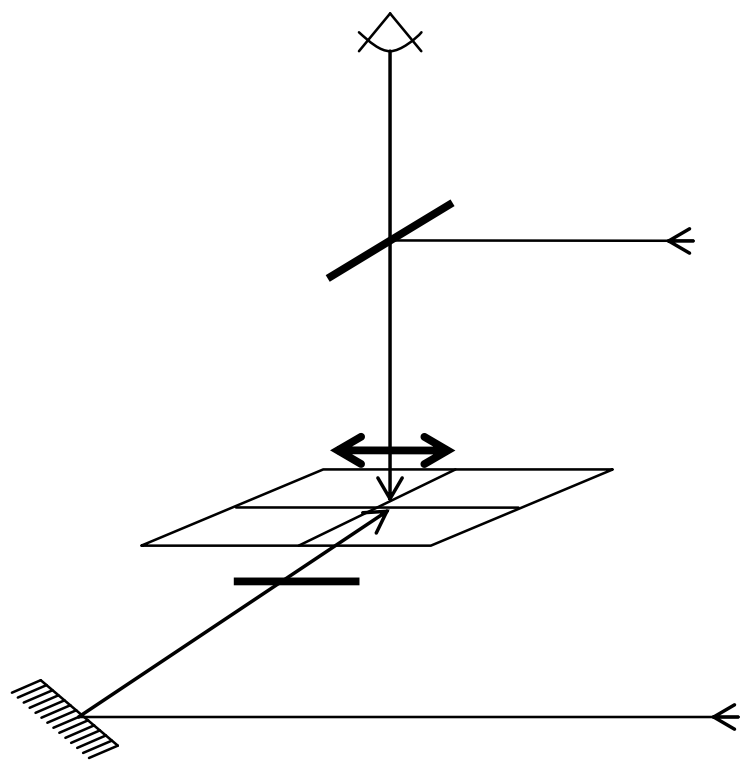

Fig. 4.10. Reference and object rays for Lippmann recording

When recording this type of holograms, precision is of great importance. A series of steps had to be followed every time to ensure a good result.

One of these steps is was to ensure that both optical paths were adjusted to have the same optical length. Experimentally, it is measured in this system as of $192 \mathrm{~cm}$ for both the reference beam and the object beam path. The entire system is compact and stable. 
Specially, the reference spot would not be a circle, because the beam hits the target from an angle different from the perpendicular, and need to be adjusted carefully to cover the object spot as shown in Fig. 4.11.

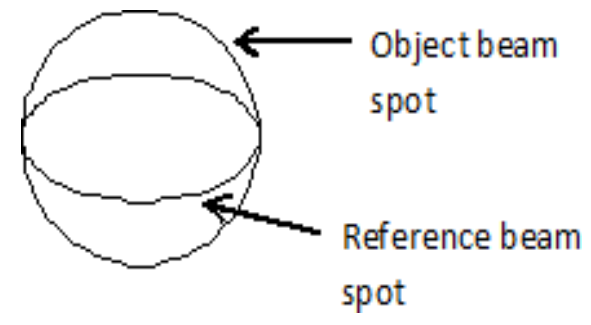

Fig. 4.11. Beams spot sizes as seen through the microscope

The pinhole used for the reference beam has a $200 \mu m$ diameter. The $\mathrm{R}, \mathrm{G}$ and $\mathrm{B}$ spots are experimentally overlapping a bit to maximum the brightness. In addition, the plate with the photosensitive emulsion must be with the emulsion facing down, to avoid the reflection noise from the glass layer by reference beam. The procedure listed below is practically programmed to achieve automatic operation for $256 \times 256$ spot.

The precise checkpoints used before the recording are enumerated here:

(1) Turn on red laser and wait for 20 min until stable

(2) Rotate to red color filter

(3) Open shutter for red color exposure, $256 \times 256$ spots

(4) Shut down red laser and turn on green laser and wait for 20 min until stable

(5) Rotate to green color filter

(6) Move the XY stage to origin point and shift $50 \mu \mathrm{m}$ meter in horizontal

Opening shutter for green color exposure, $256 \times 256$ spots

(7) Shut down green laser and turn on blue laser and wait for 20 min until stable

(8) Rotate to blue color filter

(9) Move the XY stage to origin and shift $50 \mu m$ meter in vertical

(10) Open shutter for blue exposure, $256 \times 256$ spots 
(11) Shut down shutter and move the XY stage to origin.

(12) Shut down the blue laser and signing for developing

The exposure energy for each color is shown as below.

\begin{tabular}{|l|l|l|l|}
\hline Color & $\begin{array}{l}\text { Reference } \\
\text { Intensity }\end{array}$ & Object Intensity & Exposure Time \\
\hline Red & 67 & 65 & $8 \mathrm{~ms}$ \\
\hline Green & 210 & 186 & $8 \mathrm{~ms}$ \\
\hline Blue & 42 & 39 & $8 \mathrm{~ms}$ \\
\hline
\end{tabular}

Table 6.1 Exposure energy

\subsection{Experimental results}

Using the system volume hologram recording mode described above, a color portrait hologram and a CG hologram were successfully synthesized as shown in Fig. 4.12. A series of $256 \times 256$ perspective images were further processed using an image processing technique for parallax views (19) (15). The element hologram size reached was $100 \mu \mathrm{m}$ in our test samples; however, up to $50 \mu \mathrm{m}$ could be experimentally achieved without seriously decreasing the diffraction efficiency.

The image processing applied really improved the 3D effect compared to the raw perspective views. It makes the full process slower, because an extra step is required on the pre-calculation phase, but doesn't alter the recording time. Not only were the $3 \mathrm{D}$ effect is more visible when the objects are moved close to the hologram plane, but the geometry used doesn't introduce any kind of aberrations or alterations to the reconstruction, because, as explained in 4.2.1, the same rays already present on the original perspective views, are reused in the recalculation.

On the other hand, the method described doesn't grow very well, because the algorithm is quadratic with respect to the image dimensions, but for the sizes used, this doesn't represent a problem. 


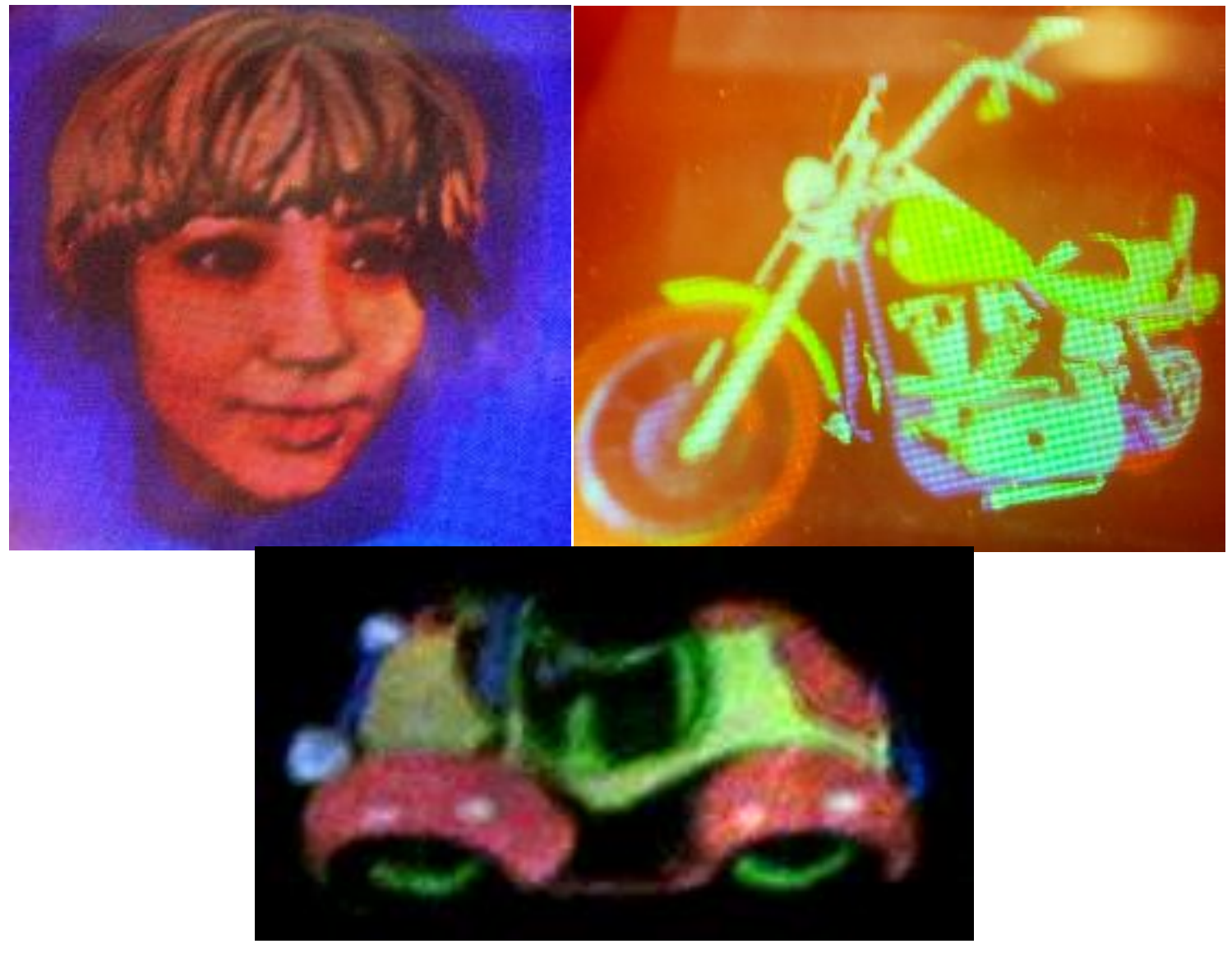

Fig. 4.12. Reflective/Lippmann parallax true-color holographic stereogram ( $4 \mathrm{~cm} \times 4 \mathrm{~cm}$ ), left: portrait image around hologram; right: CG bike image around hologram, below: CG car image behind of hologram. All of them are processed with imaging positioning technique and compensated the lens aberration, described above, respectively. 


\section{Discussion}

The experimental samples for digital relief type (rainbow) hologram and volume type (Lippmann) hologram obtained in this work are recognizable and acceptable; however, there are still some points to be improved to achieve the final goal: a hologram printer.

For the digital relief type hologram recording mode:

1. Brightness: Only about $15 \%$ - 20\% diffractive efficiency (D.E.) was obtained in the relief type hologram. This is due to a relief depth-distribution-control issue through exposure and etching processes. According to the theory of relief type grating, the maximum efficiency reachable with this material is about $40 \%$. The D.E will be decreased by, for example multi-exposure, or improper exposure condition, like vibration, over/less exposure, over/less developing time, etc. In this system, we limited the photoresist layer coating to a thick thinner than $0.5 \mu m$ in order to get a bright reflection from the back plate. This help to focus the system easily. But also leads to a risk of over developing of the resist layer. An improved linear-response-control process technique is expected in a future work.

2. Recording speed: The current recording speed in a digital rainbow type recording mode is about 3 hours $/ \mathrm{cm}^{2}$. The prime limitation of the recording speed is the XY stage transportation and waiting time between each element hologram exposure. The current exposure time is about $50 \mathrm{~ms}-100 \mathrm{~ms}$, but it takes an extra $100 \mathrm{~ms}$ waiting for the next location being set. Therefore, a non-stop linear XY stage or/and a bigger exposure spot is a conceivable direction for future improvement. For this latter case, a bigger SLM and larger aperture of the optical setup is required.

3. $\mathrm{CGH}$, Kinoform optimization: a big advantage of applying this system is the prospect to create any wavefront for developing different optical devises, consequently a phase only CGH or Kinoform is one of the options. It is difficult, however, to control an ideal relief profile so that the diffractive efficiency as well as the $\mathrm{S} / \mathrm{N}$ (signal to noise ratio) is still a big issue. A more detailed and much better dynamic range control is necessary for practical application. 
For the volume type hologram recording mode:

1. Brightness: The ideal Diffractive Efficiency in volume type hologram is close to $99 \%$ while using a thick photopolymer material. In our first experiment, we used ultra-high resolution particle material with $15 \mu \mathrm{m}$ thickness that achieves close to a maximum $80 \%$ efficiency due to the scattering effect from the particle components of the material, but practically only about 40$50 \%$ was obtained in our results. The main reason can be the system vibration during exposure and the energy distribution of a single exposure spot surpassing the dynamic range of the material.

2. For the former issue, it is necessary to make the whole system structure firm. This can be achieved through reducing the optical path and decreasing the exposure time. As for the latter issue, we consider applying a light random phase control on the second SLM in the system for better dynamic range control.

3. Recording speed: The current speed for automatically synthesizing a $4 \mathrm{~cm} \times 4 \mathrm{~cm}$ full color stereogram is about 18 hours. It is possible to enhance the speed up by using a high power laser to reduce the exposure time.

4. Resolution: The full color Lippmann type holographic stereogram image resolution is dependent on the element size that is about $100 \mu \mathrm{m}$ (partial multi-exposure in Red, Green and Blue spots) in the initial results, however, it is still possible to make a higher resolution result by adjusting the dynamic range control as mentioned. 


\section{Conclusions}

According to the final goal of this research, that was to build a high speed hologram printer, we have proposed and practically built a prototype system. Experimental results show that our concept is capable to achieve our goal.

Because a basic optical resolution of up to $0.4 \mu \mathrm{m}$ was achieved in the basic optical setup, many different kind of diffractive optical device including the so-called 3D color rainbow type hologram, CGH, Kinoform, directional diffuser, micro-text/logo, etc., are automatically recorded through a turn-key control. The image resolution is up to $0.8 \mu \mathrm{m}$ in $\mathrm{CGH}$, and $1500 \mathrm{dpi}$ in full color 3D images with speckle noise free. The others effects, like micro-text/log, etc., achieve up to 25000 dpi resolution. It is clear that much more effects are possibly to develop through new software and plugged and merged into existing effects to create new optical devices.

In the thick Lippmann holograms, a full parallax true-color stereogram was automatically synthesized in a one step process that includes an image processing to enhance the image quality. It took about 18 hours to make a $4 \mathrm{~cm} \times 4 \mathrm{~cm}$ size with $100 \mu \mathrm{m}$ element hologram and achieve up to 60 degree viewing angle. It is even easier to make a large format full parallax holographic stereogram with bigger spot size.

Up to now, we could successfully make the same sample to realize the basic capabilities of applying the system as a real printer. It is considerable to apply the results in security or diffractive optical device applications, but still there is some needs to improve the printing speed and automatic material developing functions. 


\section{Activities}

As part of the work for my PHD, the following activities and publications were done:

(1) Poster presentation in SPIE ( San Jose, CA, USA): Holographic data calculating algorithm and new digital hologram recorder, Maria-Luisa Cruz-Lopez, Miguel Alcaraz-Rivera, J. Javier Baez-Rojas, and Der-Kuan Kang, Proc. SPIE 6488, 648810 (2007).

(2) Oral presentation in SPIE ( San Jose, CA, USA): Development of a fully functioning digital hologram system, Miguel Alcaraz-Rivera, J. Javier Baez-Rojas, and Kang Der-Kuan, Proc. SPIE 6912, 69120S (2008).

(3) Journal paper: New fully functioning digital hologram recording system and its applications, DerKuan Kang, Miguel Alcaraz Rivera, Javier Baez, and Maria Luisa Cruz-Lopez, Opt. Eng. 49, 105802 (2010). 


\section{Appendix}

\subsection{Diffraction Efficiency for a profile grating}

\subsubsection{Grating function of a profile grating}

Any periodic function $x(t)$ can be presented as following equation,

$$
x(t)=\frac{a_{0}}{2}+\sum_{n=1}^{\infty}\left[a_{n} \cos \frac{2 \pi n t}{T}+b_{n} \sin \frac{2 \pi n t}{T}\right]
$$

Here, $\omega=\frac{2 \pi}{T}$ and $n=n^{\text {th }}$ period function case.

We can use, $\cos \theta=\frac{e^{i \theta}+e^{-i \theta}}{2 i}$

Substitute into equation we have,

8.1

$$
x(t)=\frac{a_{0}}{2}+\sum\left(a_{n} \cos n \omega_{0} t+b_{n} \sin n \omega_{0} t\right)
$$

Because $\cos n \omega_{0} t=\frac{e^{i n \omega_{0} t}+e^{-i n \omega_{0} t}}{2}$ and $\sin n \omega_{0} t=\frac{e^{i n \omega_{0} t}-e^{-i n \omega_{0} t}}{2 i}$.

Substitute into equation

\section{2}

We have: $x(t)=\frac{a_{0}}{2}+\sum_{n=1}^{\infty} \frac{a_{n}-i b_{n}}{2} e^{i n \omega_{0} t}+\sum_{n=1}^{\infty} \frac{a_{n}+i b_{n}}{2} e^{-i n \omega_{0} t}$

Where $C_{0}=\frac{a_{0}}{2}, C_{n}=\frac{a_{n}-i b_{n}}{2}, C_{-n}=\frac{a_{n}+i b_{n}}{2}$

So:

$$
x(t)=C_{0}+C_{n} e^{i n \omega_{0} t}+\sum_{n=1}^{\infty} C_{-n} e^{-i n \omega_{0} t}
$$


If substitute $-n$ for $n$ in the $3^{\text {rd }}$ item

$$
\sum_{n=1}^{\infty} C_{-n} e^{-i n \omega_{0} t}=\sum_{n=-1}^{-\infty} C_{n} e^{-i n \omega_{0} t}=\sum_{n=-\infty}^{-1} C_{n} e^{-i n \omega_{0} t}
$$

Equation becomes:

8.3

$$
x(t)=C_{0}+\sum_{n=1}^{\infty} C_{n} e^{i n \omega_{0} t}+\sum_{n=-\infty}^{-1} C_{n} e^{i n \omega_{0} t}
$$

Actually when, $n=0$, equation

$$
\begin{gathered}
\text { becomes } C_{0} \text {, therefore, } \\
8.4 \\
\sum_{n=-\infty}^{\infty} C_{n} e^{i n \omega_{0} t}=\sum_{n=1}^{\infty} C_{n} e^{i n \omega_{0} t}+C_{0}+\sum_{n=-\infty}^{-1} C_{n} e^{i n \omega_{0} t}
\end{gathered}
$$

Thus

$$
x(t)=\sum_{n=-\infty}^{\infty} C_{n} e^{i n \omega_{0} t}
$$

Here $C_{n}$ means the $n^{\text {th }}$ period function and $\omega=\frac{2 \pi}{T}, \mathrm{~T}=$ cycle of the period function. How to decide $C_{n}$ from equation 8.6 ? First of all, let's say

$$
C_{n}=\frac{1}{T} \int_{0}^{T} x(t) e^{-i n \omega_{0} t} d t
$$

and $C_{k}$ is our target to resolve, from equation

we have,

$$
\frac{1}{T} \int_{0}^{T} \sum_{n=-\infty}^{\infty} C_{n} e^{i n \omega_{0} t} e^{-i k \omega_{0} t} d t=\frac{1}{T} \sum_{n=-\infty}^{\infty} \int C_{n} e^{i(n-k) \omega_{0} t} d t
$$

When $n=k$, from equation

$$
\frac{1}{T} \int_{0}^{T} x(t)^{-i n \omega_{0} t} d t=\frac{1}{T} \int_{0}^{T} C_{k} e^{0} d t=\frac{C_{k}}{T} \int_{0}^{T} d t=C_{k},
$$


When $n \neq k$, from equation

\section{8}

$\frac{1}{T} C_{n} \int_{0}^{T} \sum_{n=-\infty}^{\infty} e^{i(n-k) \omega_{0} t} d t=\left.\frac{C_{n} e^{i(n-k) \omega_{0} t}}{T i(n-k) \omega_{0}}\right|_{0} ^{T}=\frac{C_{n}}{T} \frac{1}{i\left(n-k \omega_{0}\right)}\left(e^{i(n-k) \omega_{0} T}-e^{i(n-k) \omega_{0} 0}\right)=0$

In summary, equation

8.7

is the answer of resolving $C_{n}$ for $n=k$.

\subsection{Diffraction Efficiency}

Using the above discussion, we can solve some special grating's D.E. (diffraction efficiency),

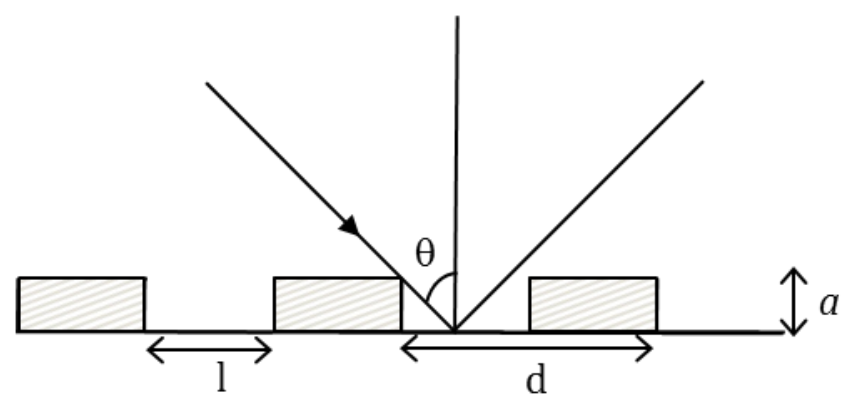

Fig. 8.1 Single ray reflecting over a grating

Assuming there is a relief grating structure as shown, the depth of the grating is $a$, the length of the grating period $d$, with a wave coming from an angle $\theta$, what would be the first order of diffraction over plane $Z_{1}$ ?

The first condition for this grating is that we assume the depth $a$ is very small compared to $d$. Thus we can easily calculate the path difference coming from the bottom and the top of the grating as: $t \cos \theta=a \therefore t=a / \cos \theta$

Path difference $=2 t=2 a / \cos \theta$

The phase before inserting plane $Z_{1}$ is $\pi$ after it is reflected from the bottom of the grating is $\frac{2 \pi}{\text { lambda }} \frac{n_{1} 2 a}{\cos \theta}+\pi$ where $n_{1}$ is the refraction index of the surface coating material. 
We have wavefront $\mu_{1}(x)$ from the grating as follows,

$$
\left\{\begin{array}{rr}
\mu_{1}(x)=e^{\frac{2 \pi}{\lambda} \frac{n_{1} 2 a}{\cos \theta}+\pi} & 0<x<l \\
\mu_{1}(x)=e^{x \pi} & l<x<d
\end{array}\right.
$$

Equation $\quad 8.9$ shows the total wavefront diffracted from the grating surface that includes $\mathrm{n}$ order diffracting wavefront.

Now we are interested in the first order diffraction assuming $S_{m}\left(Z_{1}\right)$ shows the $m^{\text {th }}$ order wavefront.

$$
\mu_{1}(x)=\sum S_{m}\left(Z_{1}\right) e^{i m \omega x}, \omega=\frac{2 \pi}{d}
$$

Using 8.7 and

to solve $S_{m}\left(Z_{1}\right)$

8.9

$$
\begin{aligned}
& S_{m}\left(Z_{1}\right)=\frac{1}{d} \int_{0} \mu_{1}(x) e^{i m \omega x} d x \\
& =\frac{1}{d} \int_{0}^{l} e^{i \frac{2 \pi}{\lambda} \frac{n_{1} 2 a}{\cos \theta}+\pi} e^{i m \omega x} d x+\frac{1}{d} \int_{0}^{l} e^{i \pi} e^{i m \omega x} d x \\
& \therefore S_{m}\left(Z_{1}\right)=\frac{1}{2 n \pi l}\left\{1-e^{i \frac{2 \pi}{\lambda} \frac{n_{1} 2 a}{\cos \theta}+\pi}\right\}\left(1-e^{i m \frac{2 \pi}{d} l}\right)
\end{aligned}
$$

Thus, the energy of the $m^{\text {th }}$ diffraction order is:

$$
\left|S_{m}\left(Z_{1}\right)\right|^{2}=\left|S_{m}\left(Z_{1}\right) S_{m}^{*}\left(Z_{1}\right)\right|=\left(\frac{2}{m \pi}\right)^{2} \sin ^{2}\left(\frac{\pi}{\lambda} \frac{n_{1} a}{\cos \theta}\right) \sin ^{2}\left(\frac{m \pi}{d} l\right)
$$

Using equation we can have two different kinds of gratings: 8.11

$$
\begin{aligned}
& d=2 l \\
& \eta=\left(\frac{2}{m \pi}\right)^{2} \sin ^{2}\left(\frac{\pi n_{1} a}{\lambda \cos \theta}\right)
\end{aligned}
$$




$$
\begin{aligned}
& d \neq 2 l \\
& \eta=\left(\frac{2}{m \pi}\right)^{2} \sin ^{2}\left(\frac{\pi n_{1} a}{\lambda \cos \theta}\right) \sin ^{2}\left(\frac{m \pi}{d} l\right)
\end{aligned}
$$

\subsection{Optimization of diffraction efficiency}

Maximizing the diffraction efficiency of the relief type hologram is a critical part in the process. It is affected by the emulsion thickness and other parameters.

From Eq. $\quad$, it is clear that conditions to achieve maximum diffraction efficiency are expressed 8.11

in Eq.

8.13

$$
d=2 l \quad \text { and } \quad a=\frac{\lambda \cos \theta}{2 n}
$$

For a typical photo-resist material with a refraction index close to 1.64 on the visible wavelength, the optimized depth $a$ is about $0.16 \mu m-0.22 \mu \mathrm{m}$. Equation 8.11 provides the optimization fringe pattern design and material etching condition for maximum efficiency in digital rainbow recording. 


\section{$4 \quad$ Image index}

FIG. 3.1 DIGITAL REPRESENTATION OF THE INTERFERENCE PATTERN OF TWO WAVE PLANES ...........................................................

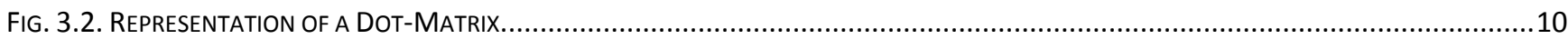

FIG. 3.3. PARAMETERS AFFECTING A HOLOPIXEL IN DOT MATRIX HologRAM PRINTING ..............................................................11

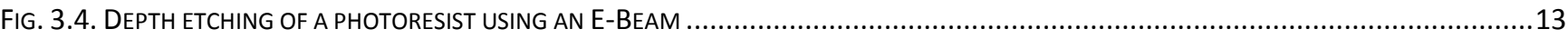

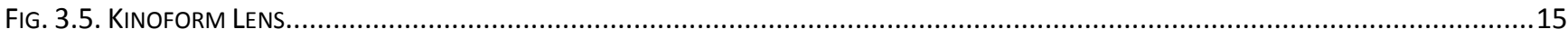

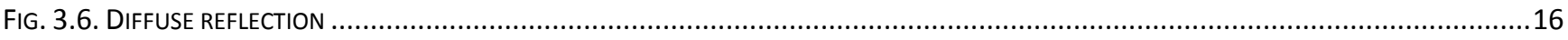

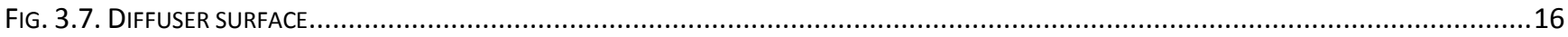

FIG. 3.8. THE MICROTEXT CAN WORK AS A DIFFUSER SCATTERING THE LIGHT IN ALL DIRECTIONS .....................................................17

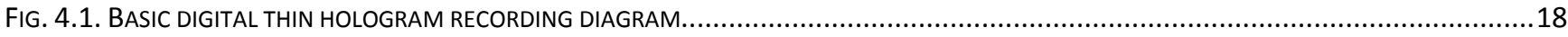

FIG. 4.2. DIAGRAM THAT SHOWS THE PARTS USED ON THE RECORDING OF A DIGITAL VOLUME HOLOGRAM .............................................20

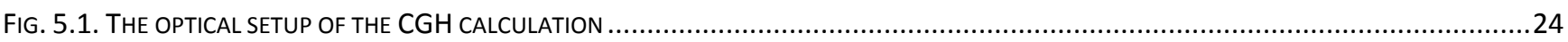

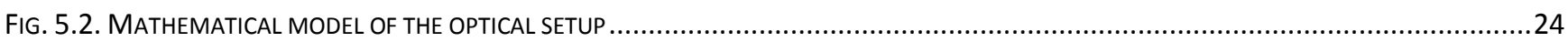

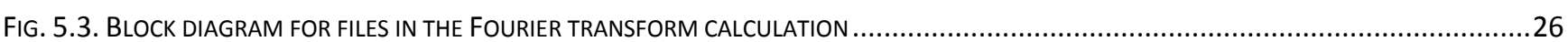

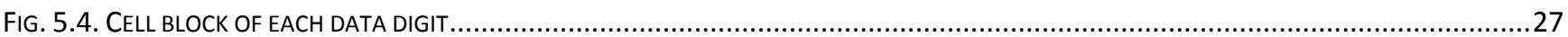

Fig. 5.5.(A)THE APERTURE $P\left(X_{1}, Y_{1}\right)$ PROVIDES PERSPECTIVES VIEWS AND WHITE LIGHT CGH IN RECONSTRUCTION (LEFT), (B) 3D COLOR IMAGE IN WHITE LIGHT PLAYBACK THROUGH ADJACENT RECONSTRUCTED APERTURE/WINDOWS (RIGHT) ....................................................37

FIG. 5.6. ANAMORPHIC LENS MODEL FOR RECORDING A 1-STEP STEREOSCOPE CGH (ABOVE) THE RECONSTRUCTED PATTERNS AT DIFFERENT

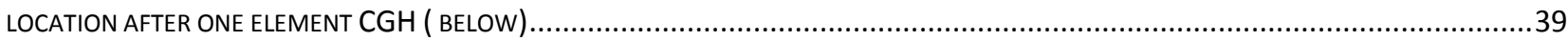

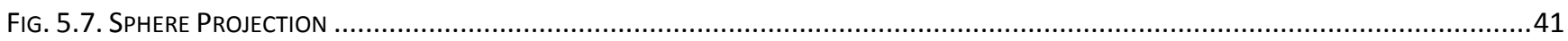

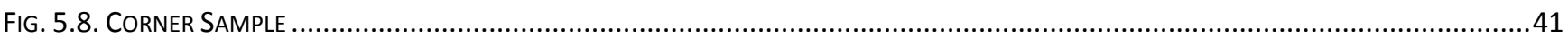

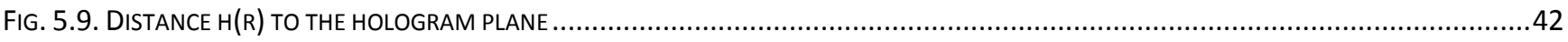

FIG. 5.10. DIAGRAM OF GRAYSCALE MAP TRANSFORMATION TO RECORDING IMAGE ......................................................................44

FIG. 5.11. EXAMPLE PATTERNS ARE CALCULATED AND DIRECTLY PROJECTED ON TO PHOTO-RESIST MATERIAL BASED ON SINGLE 2D MAP (G). FROM TOP-LEFT CLOCKWISE DIRECTION; (A) BLAZED GRATING (B) NON-DIFFRACTIVE MICRO-TEXT (C) DIRECTIONAL DIFFUSER (D) MICRO-LENS (E)

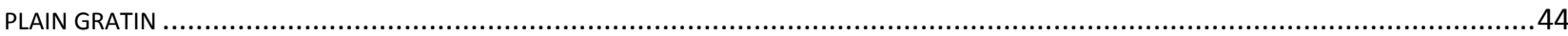

FIG. 5.12. EXPERIMENTAL SAMPLE THAT CONSISTS OF VARIOUS OPTICAL DATA DIRECTLY PRINTED THROUGH SINGLE WELL-DESIGNED 2D GRAYSCALE

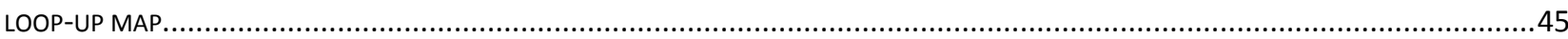

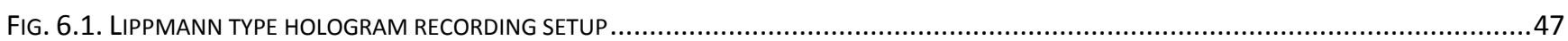

FIG. 6.2 HORIZONTAL ONLY IMAGE PROCESSING HOLOGRAPHIC STEREOGRAM (LEFT), PARALLAX VIEW IMAGE PROCESSING (RIGHT).....................48

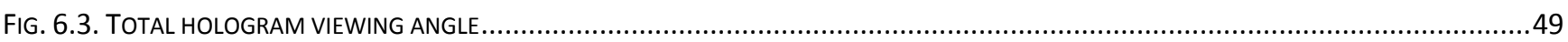

FIG. 6.4. A CERTAIN POINT INSIDE THE OBJECT IS VIEWED ONLY IN A CERTAIN PART OF THE HOLOGRAM ...............................................50

FIG. 6.5 AN EXAMPLE OF ORIGINAL PERSPECTIVE IMAGE (COKE CAN, LEFT), AFTER PROCESSED USING THE NEW ALGORITHM (RIGHT) WHICH CALCULATION IS MUCH FASTER AND MORE FLEXIBLE THAN TRADITIONAL METHOD ..............................................................51

FIG. 6.6. A CURVED SURFACE CHANGES THE OBJECT SEGMENT SEEN FROM A CERTAIN POINT ..........................................................51

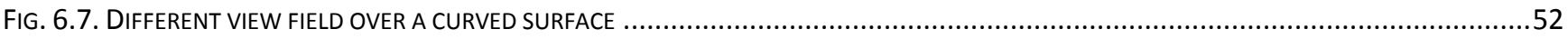


Fig. 6.8 INPUT IMAGES (A) AND (C), PRE-DISTORTED IMAgES (B) AND (D) FOR COMPENSATING THE LENS ABERRATION .54

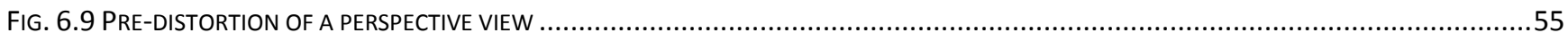

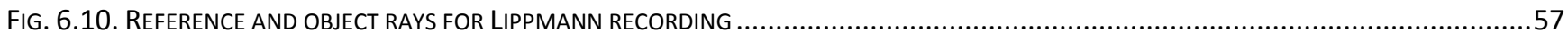

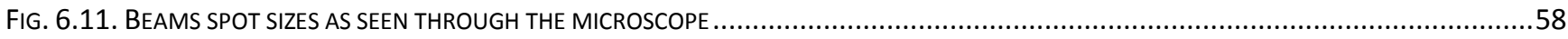

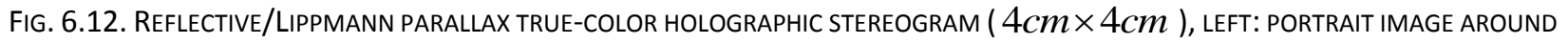
HOLOGRAM; RIGHT: CG BIKE IMAGE AROUND HOLOGRAM, BELOW: CG CAR IMAGE BEHIND OF HOLOGRAM. ALL OF THEM ARE PROCESSED WITH IMAGING POSITIONING TECHNIQUE AND COMPENSATED THE LENS ABERRATION, DESCRIBED ABOVE, RESPECTIVELY.......................60

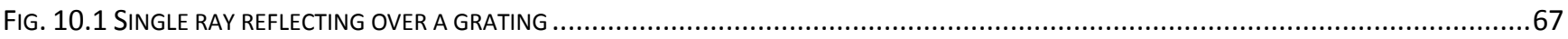




\section{Bibliography}

1. Ackermann, Gerhard K. and Eichler, Jürgen. Holography: A Practical Approach. s.1. : WILEYVCH, 2007.

2. Hecht, Eugene. Optics. s.l. : Addisson Wesley, 2000.

3. Reconfigurable image projection holograms. Plesniack, W, et al., et al. 115801, s.1. : Opt. Eng., 2006, Vol. 45(11).

4. Davis, Frank S. Holographic image conversion method for making a controlled holographic grating. 5,262,879 US, Nov. 16, 1993.

5. Dot matrix technique for generating diffraction grating patterns. Newswanger, C. p. 307, s.1.: Proceedings of SPIE, Vol. 2333.

6. Newswanger, C. Holographic diffraction grating patterns and methods for creating the same. 5,291,317 US, Mar 1, 1994.

7. Theoretical and experimental study of dot matrix hologram. Yaotang Li, Tianji Wang, Shining Yang, Shichao Zhang, Shaowu Fan, and Huanrong Wen. 121, s.1. : Proc. SPIE, 1998, Vol. 3559.

8. The kinoform: a new wavefront reconstruction device. L. B. Lesem, P. M. Hirsch, and J. A. Jordan. s.l. : IBM J. Res. Develop., 1969, Vol. 13, pp. 150-155.

9. Method for Computing Kinoforms that Reduces Image Reconstruction Error. Liu, N. C. Gallagher and B. s.1. : Appl. Opt., 1973, Vol. 12, pp. 2328-2335.

10. Grating image technology. Iwata, F. s.1. : Proc. SPIE, 1995, Vol. 2577, pp. 66-70.

11. Grating images. Ohuma, F. Iwata and K. s.1. : Proc. SPIE, 1988, Vol. Optical Security Systems, pp. 12-14.

12. Expanding a color presentation range with true-color dor matrix holograms. Tu, C.-W., Han, Y.A. and Lee, C.-K. s.1. : Proc. SPIE, 1999, Vol. 3637, pp. 130-140.

13. Electron beam fabrication of computer-generated holograms. Arnold, S. M. s.1. : Opt. Eng., 1985, Vol. 24(5), pp. 803-807 . 
14. Holographic data calculating algorithm and new digital hologram recorder. M. Cruz-Lopez, M. Alcaraz, J. Baez-Rojas, and D.-K. Kang. 648810, s.1. : Proc. SPIE , 2007, Vol. 6488.

15. Large one-step holographic stereogram. Honda, D.-K. Kang, K. Shimura, H. Enomoto, M. Yamaguchi, and N. Ohyama. s.1. : Proc. SPIE, 1991, Vol. 1461, pp. 156-166.

16. The kinoform: a new wavefront reconstruction device. LB Lesem, PM Hirsch, and JA Jordan, Jr. s.l. : IBM J. Res. Dev., 1969, Vol. 13, pp. 150-155.

17. Computer generated rainbow hologram. Taniguchi, H. Yoshikawa and H. s.l. : Opt. Rev., 1999, Vol. 6(2), pp. 118-123.

18. Phase-added stereogram: calculation of hologram using computer graphics technique. M. Yamaguchi, H. Hoshino, T. Honda, and N. Ohyama. s.1. : Proc. SPIE, 1993, Vol. 1914, pp. $25-33$.

19. One-step rainbow hologram. Yu, H. Chen and F. T. S. s.l. : Opt. Lett., 1978.

20. P., Hariharan. Optical holography. s.1. : Cambridge University Press, 1984.

21. Poon, Ting-Chung. Digital Holography and Three-Dimensional Display. s.1. : Springer, 2006.

22. On a method for reducing the information content of holograms. Benton, S.A. 1545, s.1. : J. Opt. Soc. A., 1969, Vol. 59.

23. Synthetic diffractive elements for security applications realized on an enhanced integral dot-matrix system. Marek Skeren, Pavel Fiala, and Ivan Richter. 27-32, s.1. : Appl. Opt. , 2006, Vol. 45.

24. Electron beam lithography-Resolution limits. al., A. N. Broers et. 131-142, s.1. : Microelectronic Engineering 32: , 1996, Vol. 32.

25. Madou, Mark, J. Coane, Philip. Introduction to Electron Beam Lithography. s.1. : Louisiana Tech University, Institute for Micromanufacturing Fundamentals of Microfabrication. pp. 41-43.

26. Electron beam lithography: Resolution limits and applications. Vieu C., Carcenac F., Pepin A., Chen Y., Mejias M., Lebib A., Manin-Ferlazzo L., Launois H.,. s.1. : Applied Surface Science, 2000, Vols. 164 (1-4), pp. 111-117.

27. Color holographic stereograms. Fusek, L. Huff and R. L. s.1. : Opt. Eng., 1980, Vol. 19(5), pp. 691-695. 
28. Eichler, G. Ackermann and J. Holography: A Practical Approach. Weinheim, Germany : WileyVCH, Verlag GmbH \& Co., 2007.

29. Expanding a color presentation range with true-color dot matrix holograms. C.-W. Tu, Y. A. Han, C.-K. Lee, J. W. Wu, A. S. T. Peng, E. H. Z. Liao,and J. T. Lee. s.1. : Proc. SPIE, 1999, Vol. 3637, pp. $130-140$.

30. High-quality recording of a full-parallax holographic stereogram with a digital diffuser. M. Yamaguchi, H. Endoh, T. Honda, and N. Ohyama. s.1. : Opt Lett., 1994, Vol. 19(2), pp. 135-137.

31. Binary computer-generated holograms. Lee, W.-H. s.1. : Appl. Opt., 1979, Vol. 18, pp. 3661-3669.

32. One-step Lippmann holographic stereogram. D.-K. Kang, T. Honda, M. Yamaguchi, and N. Ohyama. 127 , s.1. : Proc. SPIE, 1992, Vol. 1667.

33. High-density recording of full-color full-parallax holographic stereogram. S. Maruyama, Y. Ono, and M. Yamaguchi. 69120N, s.1. : Proc. SPIE, 2008, Vol. 6912. 